Portland State University

PDXScholar

$7-27-2020$

\title{
Guided Reinvention as a Context for Investigating \\ Students' Thinking about Mathematical Language and for Supporting Students in Gaining Fluency
}

Kristen Vroom

Portland State University

Follow this and additional works at: https://pdxscholar.library.pdx.edu/open_access_etds

Part of the Mathematics Commons, and the Science and Mathematics Education Commons Let us know how access to this document benefits you.

\section{Recommended Citation}

Vroom, Kristen, "Guided Reinvention as a Context for Investigating Students' Thinking about Mathematical Language and for Supporting Students in Gaining Fluency" (2020). Dissertations and Theses. Paper 5546.

https://doi.org/10.15760/etd.7420

This Dissertation is brought to you for free and open access. It has been accepted for inclusion in Dissertations and Theses by an authorized administrator of PDXScholar. Please contact us if we can make this document more accessible: pdxscholar@pdx.edu. 
Guided Reinvention as a Context for Investigating Students' Thinking about Mathematical Language and for Supporting Students in Gaining Fluency

by

Kristen Vroom

A dissertation submitted in partial fulfillment of the requirements for the degree of

Doctor of Philosophy

in

Mathematics Education

Dissertation Committee:

Sean Larsen, Chair

John Caughman

Joanna Bartlo

Mark Yannotta

Andrea Goforth

Portland State University

2020 
(C) 2020 Kristen Vroom 


\begin{abstract}
Fluency with mathematical language is important for students' engagement in many disciplinary practices such as defining, conjecturing, and proving; yet, there is growing evidence that mathematical language is challenging for undergraduate students. This dissertation study draws on two design experiments with pairs of students who were supported to encode their mathematical meanings with more formal language. I aimed to investigate the teaching and learning of mathematical language, and particularly the language in statements with multiple quantifiers, by engaging students in this type of activity. In the first paper, I investigated the complex ways in which the students in my study made sense of the grammar of statements with multiple quantifiers. This study contributes to the research literature in two ways. First, I showcased grammatical features that influenced the ways that students constructed and interpreted these statements. Second, I complemented the current static descriptions of statements with multiple quantifiers found in the literature with a dynamic understanding of these statements, which grew out of exploring the students' thinking. The second paper is a case study of students reinventing the dynamical processes that are encoded with statements with multiple quantifiers. This paper offers a proof of concept of an instructional approach that engages students in defining multiple concepts and then reflecting on the resulting set of definitions to learn about the grammar of statements with multiple quantifiers. This paper also takes initial steps toward articulating a local instructional theory that frames the students' reinvention process in terms of the emergent models construct from the theory of Realistic Mathematics Education. In the third paper, I explored the teachers' role in
\end{abstract}


supporting students in encoding their mathematical meanings with more formal language as the students engaged in defining and conjecturing. I used the conceptualization of the teacher's role as a broker between the local community with students and the broader mathematics community. I offer episodes of brokering that supported students in refining their language. This paper's contribution is a conceptual framework of brokering by leveraging pedagogical content tools and intellectual needs to support students in progressive mathematizing. 


\section{Acknowledgements}

There are many people that have contributed to my intellectual growth and personal well-being throughout my doctoral studies. I am incredibly grateful for each and every one of you.

I cannot thank my advisor, Sean Larsen, enough for his mentorship. What I can put into words is insufficient for my admiration for you as a researcher and mentor. Thank you for deeply engaging with my thinking and giving me feedback that supported me to refine my ideas. I am honored to be one of your students and I aspire to give my own future students a similar experience. Also, thank you to the other members of my committee. I appreciate your thoughtful feedback and your willingness to devote your energy into supporting me through this process.

Thank you to my husband, Sam, for your love and support. Thank you for helping me to understand that my big feelings during this experience were reasonable, and a necessary component of this process, because I was accomplishing a big thing. I can always count on your logical perspective to remind me how far I have come as evidence for how far I can go. Thank you to the rest of my family and friends for cheering me on. Thank you to my parents for teaching me to value my education, and for supporting me to pursue a career that I love. Ashley and Emmy, I will forever cherish our daily Facetime calls for my much-needed writing breaks.

I belong to several communities of learners that have inspired and encouraged me throughout my doctoral studies. A special thank you to the Advanced Calculus Super Team and the larger ASPIRE project team for your collaboration. My favorite part of 
academia, as I know it, is learning with you. Thank you for reading with me, being curious with me, and for pursuing research questions with me. Also, thank you for my (crazy cool) cohort for our friendship and for being a support system throughout our doctoral program. I greatly appreciate the fellow graduate students who helped me to collect my data and/or took time to read my papers. Tenchita, Bea, David, and Brenda thank you for not only reading drafts but for always being willing to have conversations with me about my work.

Last, but certainly not least, thank you to my participants. It was an honor to work with you. I have learned so much from you and sincerely appreciate the generosity that you displayed with sharing your thinking. Your mathematical creativity is truly inspiring. 
Table of Contents

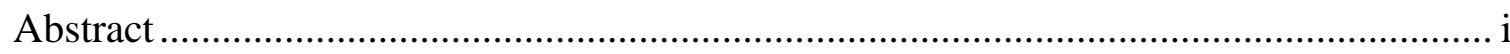

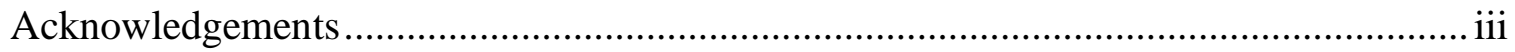

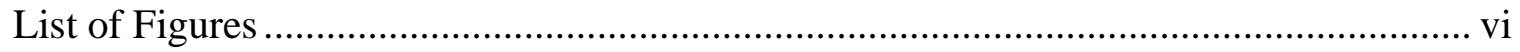

Chapter 1: Introduction .................................................................................... 1

Chapter 2: Students' mathematical language: Differences that make a difference .......... 13

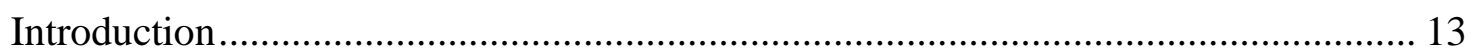

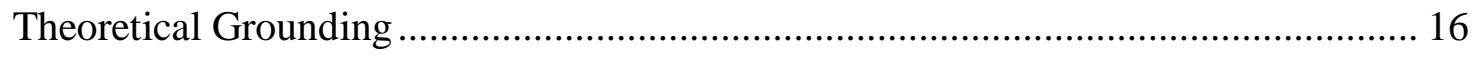

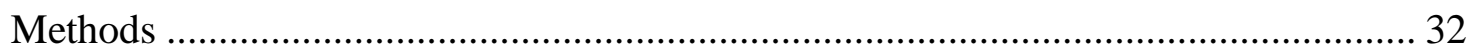

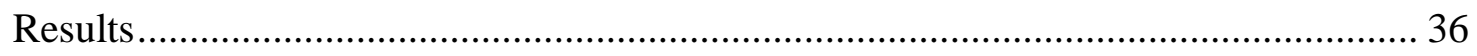

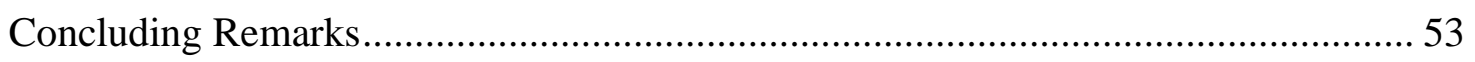

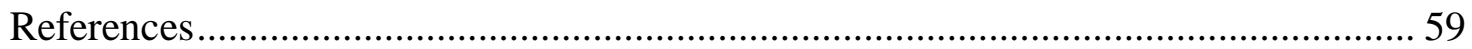

Chapter 3: A Case Study of Gaining Fluency with Mathematical Language by way of

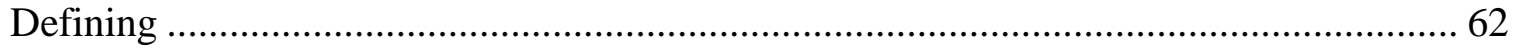

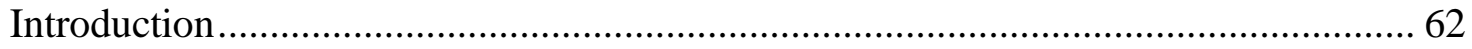

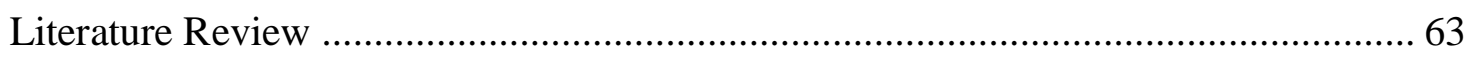

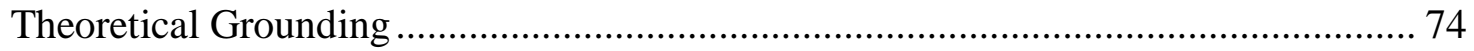

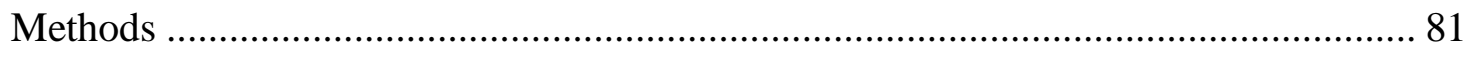

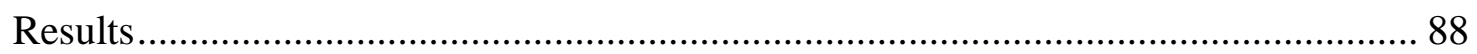

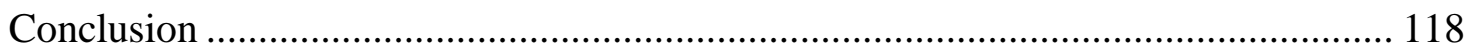

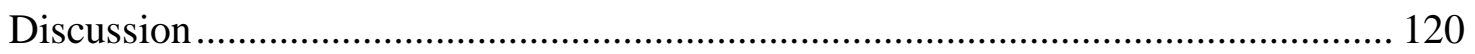

References................................................................................................... 123

Chapter 4: The Role of Brokering as Students Engage in Mathematical Statement Writing

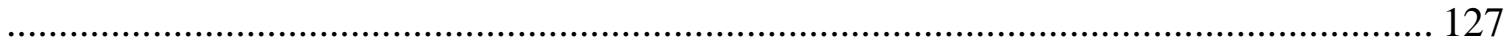

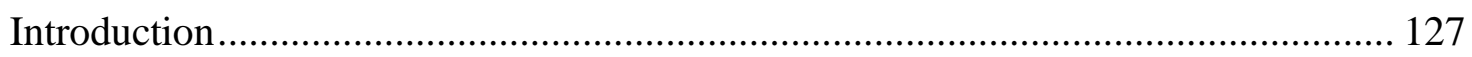

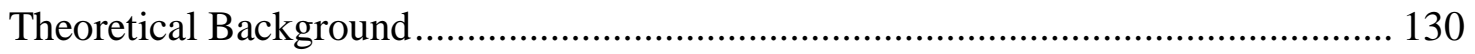

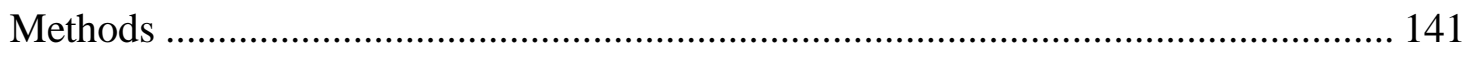

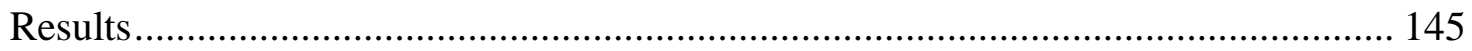

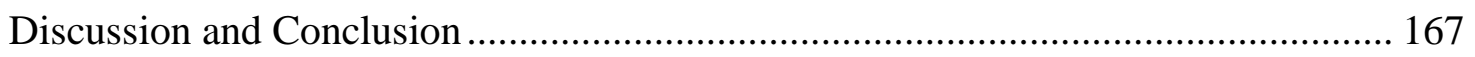

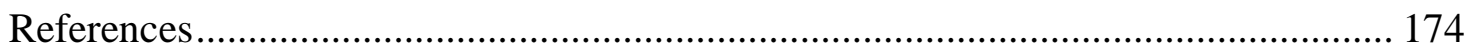

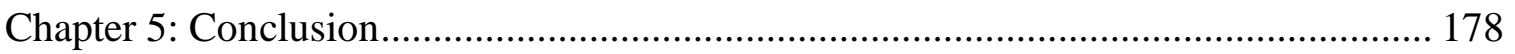

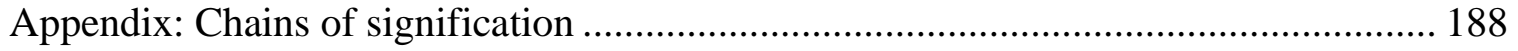

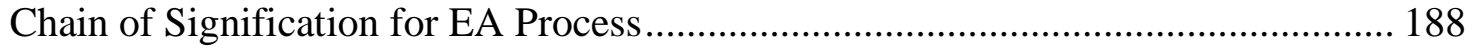

Chain of Signification for AE Process .............................................................. 192 


\section{List of Figures}

Figure 1. Pairwise relationships (adapted from Dawkins \& Roh, 2019, p. 4)................ 23

Figure 2. Examples of each-to-some relationships...................................................... 25

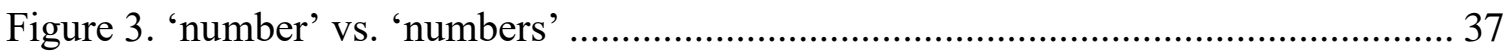

Figure 4. Problem with Ada and Lori’s proposed definition. ......................................... 38

Figure 5. Prime Sequence definition with 'for every' vs. 'for all' ................................. 39

Figure 6. Using 'for all' to construe one-to-all meaning. .......................................... 41

Figure 7. Proposed property for Prime Sequence definition.......................................... 43

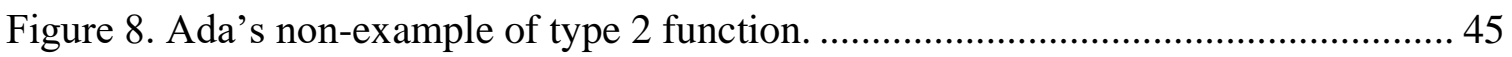

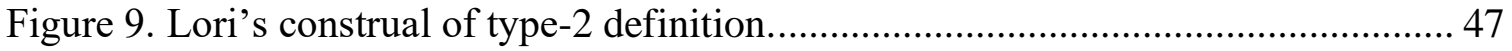

Figure 10. Eventually Constant Sequence definition .................................................... 48

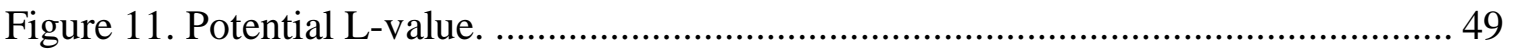

Figure 12. Lori's illustrations of Star interpretations................................................. 53

Figure 13. Invalid proof that $1 \mathrm{n}$ did not converge to 0 (from Dawkins \& Roh, 2016, p.

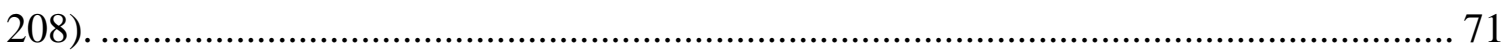

Figure 14. The Mayan stonecutter story (from Roh \& Lee, 2011, p. 694)..................... 72

Figure 15. Six principles of model-eliciting activities............................................ 81

Figure 16. Generation of Eventually Constant Sequence. ............................................ 90

Figure 17. Examples and non-examples of Eventually Constant Sequence.................... 91

Figure 18. The location of L with an Eventually Constant Sequence ............................. 94

Figure 19. A "momentarily constant" sequence. ......................................................... 95

Figure 20. A table of definition refinements for Eventually Constant Sequence. ............ 98

Figure 21. Examples and non-examples of Prime sequence....................................... 100

Figure 22. Issue with 'There exists at least one input $k$ for all prime numbers in P' ..... 103

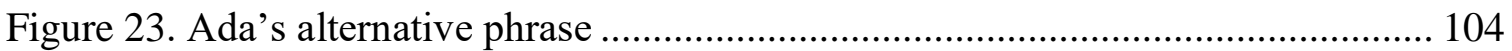

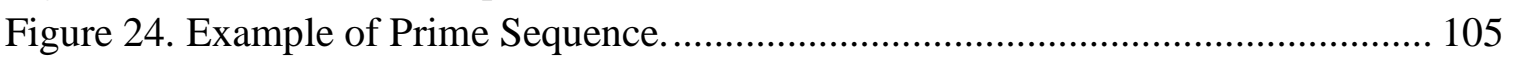

Figure 25. A table of definition refinements for Prime Sequence. .............................. 108

Figure 26. Statements provided for Ada and Lori to aid them in writing instruction

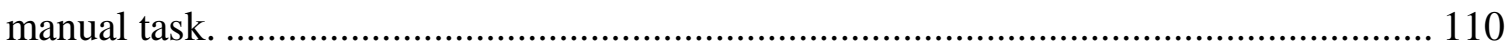

Figure 27. Lori's illustrations of Star interpretations..................................................... 111

Figure 28. 'There exists a for every b in B' versus 'There exists at least one a for every b in B'

Figure 29. Illustration of Prime Sequence …......................................................... 113

Figure 30. Lori’s red objects-and-slider analogy .................................................. 115

Figure 31. Ada and Lori's instruction manual ......................................................... 117

Figure 32. Chloe and Gabe's definition of "upper bound"........................................ 146

Figure 33. Chloe and Gabe's attempt at defining "no upper bound"............................. 146

Figure 34. Gabe's illustration of an unbounded above sequence. ............................... 147 
Figure 35. Refined definition of "no upper bound".

Figure 36. Brokering to support students engaging in disciplinary practices: Episode 1.

Figure 37. Brokering to support students engaging in disciplinary practices: Episode 2 and 3.

Figure 38. Conceptual Framework for Brokering to Support Students' Progressive Mathematizing

Figure 39. Dynamic processes of EA and AE statements. 179 Figure 40. Conceptual Framework for Brokering to Support Students' Progressive Mathematizing. 


\section{Chapter 1: Introduction}

Halliday's (1978) notion of register, and particularly mathematics register, is fundamental in the study of mathematics as a language. A register is, "a set of meanings that is appropriate to a particular function of language, together with the words and structures which express these meanings" (Halliday, 1978, p. 195) and the mathematical register is "the meanings that belong to the language of mathematics (the mathematical use of natural language, that is: not mathematics itself), and that a language must express if it is being used for mathematical purposes" (Halliday, 1978, p. 195). The mathematical register features semiotic systems (e.g. symbolic notation, visual displays) and grammatical patterns (e.g. technical vocabulary, implicit logical relationships) which are used to convey technical and precise meanings (Schleppegrell, 2007).

Researchers have argued that undergraduate mathematics students in proof-based courses should engage in mathematical practices that parallel the activity of mathematicians (e.g. Larsen \& Zandieh, 2008; Melhuish et al., 2020; Rasmussen et al., 2015). Defining and conjecturing are core to mathematicians' work (Rasmussen et al., 2005; Rasmussen et al., 2015) and include using mathematical language to specify meanings. Students, in comparison to mathematicians, experience an added complexity when engaging in defining or conjecturing. There is growing evidence that formal mathematical language is challenging for undergraduate students (Dawkins \& Roh, 2019;

Dubinsky \& Yiparaki, 2000, Lew \& Mejía-Ramos, 2015, 2019).

This dissertation study draws on data from two design experiments (Cobb and Gravemeijer, 2008) with pairs of students who were guided to reinvent a variety of 
definitions and theorems. The instructional approach leveraged the guided reinvention heuristic from the theory of Realistic Mathematics Education. The heuristic maintains that instruction should "allow learners to come to regard the knowledge they acquire as their own, personal knowledge, knowledge for which they themselves are responsible" (Gravemeijer \& Terwel, 2000, p. 786). The students in my study regularly engaged in writing mathematical statements with multiple quantifiers to articulate their mathematical meanings. In line with guided reinvention, the students typically wrote their mathematical idea informally and then the students and teacher-researchers would work to refine their language toward a more conventional, valid, and logical form. My research goal for my dissertation study was to investigate (a) the students' thinking about mathematical language as they engaged in trying to use it to encode their mathematical thinking, and (b) how engaging students in encoding their mathematical thinking with more formal language can be leveraged to support them in gaining fluency with formal mathematical language.

\section{Context of Study}

My dissertation project is part of a larger design research project (Cobb \& Gravemeijer, 2008) that has evolved into a project supported by the National Science Foundation, Advancing Students' Proof Practices in Mathematics through Inquiry, Reinvention, and Engagement (NSF DUE \#1916490)1. In what follows, I will describe 
the evolution of the project in relation to my involvement, interests, and motivation for my dissertation research.

I joined the project team when I entered my doctoral program. At this point, we were continuing to build on Strand's (2016) dissertation work, designing a local instructional theory in the context of real analysis. A local instructional theory is a generalized sequence of instructional activities with a rationale for the choice of activities (Gravemeijer, 1998). Our design was guided by the theory of Realistic Mathematics Education, and particularly, the heuristic of didactical phenomenology (Larsen, 2018). Larsen explains that one of the first steps to design a local instructional theory is to engage in a thought experiment through reflecting on how such a concept might be reinvented. Investigating how the pioneers of the targeted concept invented such a concept is often a rich resource in this process. In this way, the instructional design was inspired by the historical development of real analysis, and particularly, Cauchy's proof of the Intermediate Value Theorem. The instructional sequence guides students as they follow Cauchy's lead by repurposing root-approximation methods to reinvent a proof of the Intermediate Value Theorem (IVT). As students engage in reinventing the proof, the students develop several fundamental concepts in real analysis such as sequence convergence, completeness, and continuity.

Grabiner's (2012) historical account of the foundations of calculus was a source of inspiration for our task design. Grabiner explained that in the nineteenth century mathematicians were called to provide explanations of the theoretical underpinnings of the calculus. Mathematicians like Cauchy, Bolzano, and Weierstrass were tasked to 
invent the foundations of calculus with the utmost rigor, clarity, and simplicity (Grabiner, 2012). That is, they needed to figure out how to rigorously, clearly, and simply prove statements that were previously taken to be true based on intuitions (e.g. the IVT). This task led the nineteenth century mathematicians studying calculus to develop the concepts in a way that would allow them to do just that. During my reading, I saw a parallel between the mathematicians inventing real analysis and the students that we were working with to support our ongoing task refinement. In particular, we were guiding the students to come up with methods to rigorously, clearly, and simply prove statements that were previously taken to be true based on their intuitions from calculus. We conjectured that our task sequence could be a rich starting point to support students in learning about proof and proof-related practices. My dissertation research, and boarder research interests, were motivated by this conjecture.

During the summer of 2018, the project team and I worked with a pair of students, Chloe and Gabe. The primary research goal for this laboratory design experiment was to refine the Intermediate Value Theorem task sequence. The secondary research goal was to test the conjecture that the students might learn about proof and proof-related practices as they engaged in the tasks. While working with Chloe and Gabe, it seemed as though this was the case, and that learning how to use mathematical language in writing definitions, conjectures, and proofs seemed like an especially fruitful research endeavor. After working with Chloe and Gabe, and expressing my interest in exploring how we could enhance the IVT local instructional theory to support students in learning about proof-related practices (particularly using mathematical language), we added this as a 
project goal. Additionally, in December 2018 we proposed the (now funded) NSF project that planned to design a course based on these principles. The project would (and currently does) design innovative instructional materials and professional development for Introduction to Proof courses. The instructional approach engages students in reinventing fundamental concepts in real analysis or group theory and then capitalizes on this experience to support students in learning about proof and proof-related mathematical practices. The real analysis materials are from the IVT local instructional theory and the group theory materials were developed during the Teaching Abstract Algebra for Understanding project (NSF DUE \#0737299).

During the Winter of 2019, we piloted our materials in a community college course that was designed to support STEM-intending students in their transition to advanced mathematics. Our instructional approach for the course was to work through the IVT task sequence, and when proof related ideas came up, we would pause the IVT sequence and hold whole class discussions about the related idea. At the end of the course, I recruited two students, Ada and Lori, to participate in a laboratory design experiment. During the experiment they engaged in tasks that I designed that explicitly leveraged the IVT task sequence and focused on supporting them in gaining fluency with mathematical language and using their definitions to structure proof.

My dissertation data comes from the two laboratory experiments with (1) Chloe and Gabe and (2) Ada and Lori. Since then, the project team has continued to refine our instructional sequences - both the IVT sequence and the task sequence that has emerged out of my dissertation project that supports students in learning about mathematical 
language. As of this dissertation's publication, the course has been offered twice in a university Introduction to Proofs course, one of which was in a remote setting due to COVID-19. The project has also piloted a remote professional development workshop. Additionally, the next iteration of the community college version of the course is currently underway.

\section{Overview of Papers}

My dissertation comes in the form of three papers. In the first paper, I investigate grammatical complexities that students considered as they constructed and interpreted mathematical statements. The second paper is a case study of two students defining multiple concepts and then reflecting on the resulting set of definitions to learn about the grammar of statements with multiple quantifiers. In the third paper, I examine the teachers' role in supporting students as they engage in mathematical statement writing to refine their language to effectively communicate their mathematical meanings.

\section{Students' Mathematical Language: Differences that Make a Difference.}

Engaging students in writing mathematical statements to communicate their own mathematical ideas gave me a unique window into students' thinking about the grammar of their statements. In my first paper, I drew on the theory of functional grammar (Halliday \& Matthiessen, 2013) to investigate grammatical differences that made a difference for the students' construed meaning of statements with multiple quantifiers. In particular, I investigated: What grammatical complexities do students consider when constructing and interpreting mathematical statements with multiple quantifiers? 
My findings agree with prior studies that students' ability to construe the conventional interpretations of statements with multiple quantifiers depends on students' understanding of the syntax (Dawkins \& Roh, 2019; Dubinsky \& Yiparaki, 2000). Unlike prior studies, I found that there were other grammatical features that influenced the students' construals other than the order of the quantifiers. Slight changes to the grammar (e.g. replacing 'for every' with 'for all', or shifting the placement of 'where') significantly changed the conveyed mathematical meaning for the students. Additionally, these complexities highlighted limitations of the ways that prior research has conceptualized statements with multiple quantifiers. In particular, the research literature has traditionally used static ways of thinking about the language in these statements and has focused on parts of the statements (i.e. the order of the quantifiers) as the defining features of the intended meaning. However, it became evident that a static view was insufficient for investigating the complex ways in which the students were making sense of the statements' grammar. I complement the static descriptions of the statements with multiple quantifiers with dynamic construals.

\section{A Case Study of Gaining Fluency with Mathematical Language by way of}

Defining. My second paper describes an instructional method designed to support students in learning about dynamical processes that are encoded with statements with multiple quantifiers. The paper is a descriptive case study of Ada and Chloe engaging in defining statements with multiple quantifiers and then reflecting on their definitions to make an instruction manual for how a peer might use statements with multiple quantifiers when defining. This approach is quite different than trying to make sense of statements 
that express other individuals' meanings that have been formally encoded using quantifiers. The important difference is that rather than working with unknown meanings and unfamiliar language rules, the students build their understanding of the syntax by trying to communicate their own meanings. This paper offers a proof of concept that students can be supported to reinvent the dynamical processes that are encoded with statements with multiple quantifiers. I also share my progress on articulating a local instructional theory by framing the students' activity as co-emerging models.

\section{The Role of Brokering as Students Engage in Mathematical Statement}

Writing. My third paper was motivated by my interests in gaining a better understanding of how an instructor might support students in refining their language. I viewed this as an important question for two reasons. First, the instructional approach that I propose in Chapter 3 relied on the students' writing definitions that used formal language. I thought that it was necessary to make progress on this question in order for the instructional sequence to work on a larger scale. Second, many mathematics educators have argued that undergraduate mathematics students in proof-based courses should engage in disciplinary practices such as defining and conjecturing (Rasmussen et al., 2015; Zandieh \& Rasmussen, 2010). However, I (as a teacher-researcher) had experienced hardships in finding ways to support students during this activity in such a way that built on their thinking toward a more conventional and more formal form. My investigation conceptualized the teacher's role as a broker between the local community with students and the broader mathematics community and investigated the brokering moves of creating boundary encounters, bringing students to the periphery, and interpreting 
between communities (Rasmussen et al., 2009). In this paper, I explored: How can teachers act as brokers to support students to refine their mathematical language when writing mathematical statements? And what is the role of intellectual needs and pedagogical content tools in this endeavor?

I present illustrative episodes of the teacher-researchers acting as brokers by how they first treated the students' statement as a boundary object and inquired into the students' thinking to detect differences between the students' meanings and the anticipated mathematics community's interpretations of the statement. Then they addressed the differences by either (a) bringing students to the periphery by evoking or leveraging intellectual needs which sometimes were supported by capitalizing on generative alternatives or (b) interpreting between communities by focusing students' attention to how the mathematics community engages in the practices. The broker then cycled through these moves until they did not detect differences between the students' meanings and the anticipated mathematics community's interpretations of the statement. While this paper focuses on brokering to support students in refining their language as they write mathematical statements, this characterization of brokering is likely to be appropriate in supporting students in engaging in other disciplinary practices. I offer a conceptual framework for brokering to support students' progressive mathematizing.

\section{Discussion}

Scholars have noted that there are limited empirical studies that investigate the mathematics register or undergraduate students' understanding and use of it (Lew \& Mejía-Ramos, 2015, 2019, 2020). The three empirical studies that comprise this 
dissertation are important steps toward such an understanding. Here, I explore students' thinking about mathematical language as they engaged in trying to use it to encode their mathematical thinking, and how engaging students in encoding their mathematical thinking with more formal language can be leveraged to support them in gaining fluency with formal mathematical language. In the following chapters, I offer my three papers. In the final chapter of this dissertation, I summarize and synthesize my findings. 


\section{References}

Cobb, P., \& Gravemeijer, K. (2008). Experimenting to support and understand learning processes. Handbook of design research methods in education: Innovations in science, technology, engineering, and mathematics learning and teaching, 68-95.

Dubinsky, E., \& Yiparaki, O. (2000). On student understanding of AE and EA quantification. Research in Collegiate Mathematics IV, 239-289.

Grabiner, J. V. (2012). The origins of Cauchy's rigorous calculus. Courier Corporation.

Gravemeijer, K. (1998). Developmental research as a research method. In Mathematics education as a research domain: A search for identity (pp. 277-295). Springer.

Gravemeijer, K., \& Terwel, J. (2000). Hans Freudenthal: A mathematician on didactics and curriculum theory. Journal of Curriculum Studies, 32(6), 777-796.

Halliday, M. (1978). Language as social semiotic: The social interpretation of language and meaning. London: Edward Arnold.

Halliday, M. \& Matthiessen, C. M. (2013). Halliday's introduction to functional grammar. Routledge.

Larsen, S., \& Zandieh, M. (2008). Proofs and refutations in the undergraduate mathematics classroom. Educational Studies in Mathematics, 67(3), 205-216.

Lew, K., \& Mejía-Ramos, J. P. (2015). Unconventional uses of mathematical language in undergraduate proof writing. In Proceedings of the 18th Annual Conference on Research in Undergraduate Mathematics Education. Pittsburgh, PA.

Lew, K., \& Ramos, J. P. M. (2020). Linguistic conventions of mathematical proof writing across pedagogical contexts. Educational Studies in Mathematics, 103(1), 43-62. 
Melhuish, K., Vroom, K., Lew, K., \& Ellis, B. (2020). Operationalizing authentic mathematical proof activity (AMPA) for the undergraduate classroom. Manuscript submitted for publication.

Rasmussen, C., Zandieh, M., \& Wawro, M. (2009). How do you know which way the arrows go? The emergence and brokering of a classroom mathematics practice. Mathematical representations at the interface of the body and culture, 171218.

Rasmussen, C., Wawro, M., \& Zandieh, M. (2015). Examining individual and collective level mathematical progress. Educational Studies in Mathematics, 88(2), 259-281.

Rasmussen, C., Zandieh, M., King, K., \& Teppo, A. (2005). Advancing mathematical activity: A practice-oriented view of advanced mathematical thinking. Mathematical Thinking and Learning, 7(1), 51-73.

Schleppegrell, M. J. (2007). The linguistic challenges of mathematics teaching and learning: A research review. Reading \& writing quarterly, 23(2), 139-159.

Strand, S. R. (2016). The Intermediate Value Theorem as a Starting Point for InquiryOriented Advanced Calculus.

Zandieh, M., \& Rasmussen, C. (2010). Defining as a mathematical activity: A framework for characterizing progress from informal to more formal ways of reasoning. The Journal of Mathematical Behavior, 29(2), 57-75. 


\section{Chapter 2: Students' mathematical language: Differences that make a difference}

Abstract: Fluency with mathematical language is necessary for students in advanced mathematics. However, little is known about students' use of mathematical language and even less about the process students undergo when learning about mathematical language. The data from this exploratory study comes from a design experiment that aimed to support students in learning about mathematical language. I found that students considered grammatical complexities as they constructed and interpreted mathematical statements with multiple quantifiers. Slight changes to the grammar (e.g. replacing 'for every' with 'for all', or shifting the placement of 'where') significantly changed the conveyed mathematical meaning for the students. These complexities highlight limitations for the ways that prior research has conceptualized statements with multiple quantifiers. I offer dynamic processes that are encoded in these statements.

Keywords: Mathematical language, mathematical statements, quantifiers, systemic functional grammar, syntax

\section{Introduction}

Halliday’s (1978) notion of register highlights that we use language to construct knowledge in different ways across different academic domains. A register is, "a set of meanings that is appropriate to a particular function of language, together with the words and structures which express these meanings" (Halliday, 1978, p. 195). In particular, a mathematical register is "the meanings that belong to the language of mathematics (the mathematical use of natural language, that is: not mathematics itself), and that a language must express if it is being used for mathematical purposes" (Halliday, 1978, p. 195). The 
mathematical register features semiotic systems (e.g. symbolic notation, visual displays) and grammatical patterns (e.g. technical vocabulary, implicit logical relationships) which are used to convey technical and precise meanings (Schleppegrell, 2007).

It is not a surprise that mathematical language is challenging for students given its technical nature. Schleppegrell's (2007) synthesis of research by applied linguists and mathematics educators gives noteworthy insight into these challenges. One challenge is that mathematical language is dense and difficult to comprehend. For instance, it uses long "noun phrases" to construct complex meaningful relationships. Schleppegrell points to Veel's (1999) example: “the volume of a rectangular prism with sides 8, 10, and 12 cm” (Schleppegrell, 2007, p. 143). In this example, 'the volume of' describes a quantifiable mathematical attribute of the prism, 'rectangular' is a classifying adjective, and 'with sides 8, 10, and $12 \mathrm{~cm}$ ' are qualifiers for the rectangular prism. Another challenge is that there are differences in the mathematics and the natural registers. For instance, mathematical language uses conjunctions like 'if', 'when', and 'then' differently (and more precisely) in mathematics than the natural language. Additionally, there are subtleties with attributive clauses in the mathematics register that do not exist in the natural register. For instance, 'a square is a quadrilateral' is not the same as 'a quadrilateral is a square' using the mathematical register, yet, the natural language does not follow the same logical rules.

Definitions, theorems, and proofs are expressed with mathematical language and are integral aspects of undergraduate mathematics courses. Students' success in these courses depends on their fluency with mathematical language. For instance, Moore 
(1994) found that students' ability to construct proofs (a typical assessment task according to Weber, 2001) depends on the students understanding mathematical language. Yet, there is growing evidence that written mathematical language is challenging for undergraduate students (e.g. Dawkins \& Roh, 2019; Dubinsky \& Yiparaki, 2000, Lew \& Mejía-Ramos, 2015, 2019). For instance, students use mathematical language unconventionally in proof writing (Lew \& Mejía-Ramos, 2015) and may not fully comprehend the nuances involved in how mathematicians introduce objects in proofs (Lew and Mejía-Ramos, 2019). Students' difficulties with statements with multiple quantifiers are especially relevant to the current study (Dawkins \& Roh, 2019; Dubinsky \& Yiparaki, 2000; Durand-Guerrier \& Arsac, 2005; Roh, 2010; Roh \& Lee, 2011). For instance, Dubinsky and Yiparaki (2000) found that students interpreted the following two statements in the same way:

AE Statement: For every positive number $a$ there exists a positive number $b$ such that $b<a$

EA Statement: There exists a positive number $b$ such that for every positive number $a$ $b<a$

Other scholars have found similar results with statements in other mathematical contexts (e.g. Dawkins \& Roh, 2019).

In order for students to participate meaningfully in advanced undergraduate mathematics classes, they must be fluent with mathematical language. Yet, the research literature has only scratched the surface of research on undergraduate students' understanding and learning of written mathematical language. The current exploratory 
study contributes to such an understanding by investigating students' construction and interpretations of mathematical statements with multiple quantifiers (e.g. defining properties, theorems). I found that students considered grammatical complexities as they constructed and interpreted mathematical statements with multiple quantifiers. Slight changes to the grammar (e.g. replacing 'for every' with 'for all', or shifting the placement of 'where') significantly changed the conveyed mathematical meaning for the students.

\section{Theoretical Grounding}

From here on, I adopt Dawkins and Roh's (2019) language regarding construal. An individual's construal of a statement (or phrase) is the meaning an individual makes for such wording. A normative construal is the construal that is taken as if shared among mathematicians, that is, the conventional interpretation of a statement or phrase.

Following a linguistic tradition, I say that individuals construe meaning from a statement or phrase to indicate that the individual attaches a particular meaning from a register to the statement or phrase.

In the following section I first discuss the normative construals of quantifiers (e.g. 'for all', 'there exists') and statements with quantifiers. This will include my reinterpretation of Dubinsky and Yiparki's (2000) game that was designed to support students in learning about statements with quantifiers as dynamic normative construals of these statements. Then, I review the research literature on students' interpretations of statements with quantifiers. Last, I introduce Halliday’s functional perspective on language (Halliday \& Matthiessen, 2013), which provides an analytical framework for 
my investigation of students' considerations of grammatical patterns and the associated construals.

\section{Normative construals of quantifiers and statements with quantifiers}

This study concerns students' construction and interpretations of mathematical statements. Mathematical statements are sentences that are either true or false and are in the mathematical context. The statements in this study make use of quantifiers, a quantified variable, and the set the quantified variable belongs to (e.g. 'for all $x \in X$ ', 'for every $y \in Y^{\prime}$ ). For instance, 'For all $x$ in the set of even numbers, $x^{2}$ is in the set of even numbers' is a true mathematical statement. Proper specifications for the variables (in terms of type and quantity) is a required characteristic of mathematical statements. For instance, 'For all $x, x^{2}$ is in the set of even numbers' is not a mathematical statement because it is ambiguous. The sentence does not specify the type of set in which $x$ holds membership and so a reader might assume $x \in \mathbb{R}$ and interpret the statement as false or they might assume $x$ is in the set of even numbers and interpret the statement as true. Additionally, 'For $x \in \mathbb{R}, x^{2}$ is in the set of even numbers' is also ambiguous. The sentence does not specify how many real numbers $x$ the statement considers. A reader

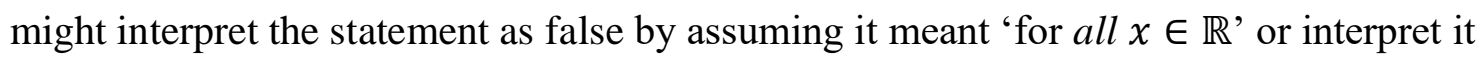
as true by assuming it meant 'for some $x \in \mathbb{R}$ '.

Quantifiers are used to specify how many of a particular mathematical object the statement considers. 'There exists' is used as an existential quantifier for a variable and 'for all' is used as a universal quantifier for a variable. When mathematicians use 'there exists' they mean 'there exists at least one' (Alcock, 2012). Mathematicians use the 
phrase 'there exists at most one' or 'there exists a unique' when it is important to convey uniqueness (Batty \& Woodhouse, 1994). There are phrases that are synonymous with 'there exists'. According to Batty and Woodhouse (1994) any of the following can replace 'there exists' in a mathematical statement: 'for some', 'for at least one', 'there is', 'has'. Also, the mathematics community sometimes symbolizes 'there exists' with ' $\exists$ '. Houston (2009) explained that when reading a statement using symbols (e.g. $\exists x \in$ $\left.\mathbb{Z}\left(x^{2}-4 x+3=0\right)\right)$ the reader should "insert a 'such that' between the quantifier and the sentence to which it refers" (p. 81). We read the above example statement as, "There exists an integer $x$ such that $x^{2}-4 x+3=0$ '. Although I found no other explicit mention of the use of 'such that' (or equivalent phrases), all of the example statements in the literature that I reviewed were consistent with this advice. For example, the following statement appeared in Dawkins and Roh's (2019) student survey (emphasis added): There exists a real number $M$ such that for all real numbers $x, f(x)<M$. The consistent use of 'such that' (or an equivalent phrase) suggests that it is common practice to use 'such that' after the existential quantifier. Other equivalent phrases include: 'for which', 'where', 'so that', 'that satisfies', 'satisfying', and 'with the property that'. Additionally, 'such that' is frequently denoted 's.t.' or ' $\exists$ '.

There was less agreement among the literature that I reviewed about the phrases that can be used as universal quantifiers. Batty and Woodhouse (1994) claim that 'for all' is synonymous with the following phrases: 'for all', 'for any', 'for every'. 'for each', 'given', and 'whenever'. Additionally, the mathematics community sometimes symbolizes 'for all' with ' $\forall$ '. However, Houston's (1994) description of universal 
quantifiers disagreed that 'for any' was equivalent to the others. He suggested that 'for any' is ambiguous, writing:

"Consider the following exercise: 'Show that $x^{2} \geq 0$ for any $x \in \mathbb{Z}$.' This can be interpreted as an instruction to show 'For all $x \in \mathbb{Z}, x^{2} \geq 0$.' However, it can also be interpreted as saying 'Choose a single integer and show that $x^{2} \geq 0$.' This is easy to do! Take $x=3$, then $x^{2}=9 \geq 0$. These two interpretations are obviously not the same yet are reasonable responses to the use of the word 'any'" (p. 81).

The literature suggests the order of the quantifiers in a statement with multiple quantifiers is the key indicator of the intended meaning (Alcock, 2012; Dawkins \& Roh, 2019; Dubinsky \& Yiparaki, 2000; Houston, 1994; Roh \& Lee, 2011). An AE statement first introduces a universally quantified variable and then an existentially quantified variable (for all $x \in X$, there exists $y \in Y$ such that $R(x, y)$ ). Whereas the variables are introduced in the opposite order in an EA statement (there exists $y \in Y$ such that for all $x \in X, R(x, y))$.

Dubinsky and Yiparki (2000) designed a two-player game to support students in understanding how one would use the order of quantifiers to make sense of the meaning of a statement. The rules of the game suggest dynamic construals of AE and EA statements. In what follows, I will review the game and then discuss its implications for how one might make meaning from an AE or EA statement.

To start the game, two players are assigned either a universal quantifier role (Player A) or an existential quantifier role (Player E) and are given an AE or EA statement. The goal of Player E is to establish that the statement is true, while Player A aims to establish that the statement is false. I will exemplify the game with four statements (from Dawkins \& Roh, 2019): 
Example 1 (True AE Statement): For every positive real number $s$, there exists a point $C$ on a ray $\overrightarrow{A B}$ such that $d(A, C)=s$.

Example 2 (False AE Statement): For every positive real number $s$, there exists a point $C$ on a segment $\overline{A B}$ such that $d(A, C)=s$.

Example 3 (True EA Statement): There exists a real number $M$ such that for all real numbers $x, \sin (x)<M$.

Example 4 (False EA Statement): There exists a real number $M$ such that for all real numbers $x, 3 x+2<M$.

In the case that the players are given an $\mathrm{AE}$ statement (e.g. Example 1 or 2), Player A takes the first turn by selecting an element from the set that the universally quantified variable belongs to (e.g. an $s$ from the set of positive real numbers) and then Player E selects an element from the set that the existentially quantified variable belongs to (e.g. a point $C$ on a ray $\overrightarrow{A B}$ or a point $C$ on a segment $\overline{A B}$ ) such that the relation is satisfied (e.g. $d(A, C)=s$ ). They continue the game by taking alternating turns. For instance, consider the game with the true AE statement example. Each time Player A takes a turn by selecting an $s$ from the set of positive real numbers, Player E will be able to find a point $C$ on the ray $\overrightarrow{A B}$ in such a way that the distance between $A$ and $C$ is the selected positive real number. Because rays extend to all positive distances away from the endpoint, the game could go on forever. Player A cannot win the game because no matter what positive real number $s$ they play, Player E will be able to find a paired $C$ such that $d(A, C)=s$. Alternatively, consider the game with the false AE statement. Since segments do not extend to all positive distances away from the endpoint, Player A can 
win the game in one turn. If Player A selects a positive real number $s$ that is greater than the length of the segment $\overline{A B}$ then Player $\mathrm{E}$ will not be able to find a point $C$ on the segment $\overline{A B}$ such that $d(A, C)=s$.

In the case that the players are given an EA statement (e.g. Example 3 or 4), Player E takes the first turn selecting an element from the set to which the existentially quantified variable belongs (e.g. an $M$ from the set of real numbers). During Player A's turn, they select an element from the set to which the universally quantified variable belongs (e.g. an $x$ from the set of real numbers) such that the relation is not satisfied (e.g. $\sin (x) \geq M, 3 x+2 \geq M)$. Consider Example 3. Since $f(x)=\sin (x)$ is bounded above, Player E can end the game in one turn and play an $M$ from the set of real numbers (e.g. $M=2$ ) so that no matter what real number Player E selects, $\sin (x)<M$. In this case, Player E will win the game. Alternatively, consider the game with Example 4. Each time Player E selects an $M$ from the set of real numbers, Player A will be able to find a real number $x$ such that $3 x+2 \geq M$. Thus, Player A will win the game since $f(x)=3 x+2$ is not bounded above.

The rules of the game have some implications for the way that Dubinsky and Yiparaki (2000) view the normative construals of AE and EA statements. In particular, I regard Player E's strategy for playing the game as analogous to construing the standard meanings of AE or EA statements. Consider the following generic AE statement, with $R(x, y)$ representing some relation between $x$ and $y$ : For all $x \in X$, there exists $y \in Y$ such that $R(x, y)$. Each time that it's Player E's turn, Player E attempts to select a $y \in Y$ for the previously played $x \in X$ such that $R(x, y)$ is satisfied. For instance, if Player A 
selects $x_{1} \in X$ then Player E selects $y_{1} \in Y$ such that $R\left(x_{1}, y_{1}\right)$ is satisfied. Then if Player A selects $x_{2} \in X$ then Player E selects $y_{2} \in Y$ such that $R\left(x_{2}, y_{2}\right)$ is satisfied. The game continues in this way. This is analogous to interpreting an AE statement as follows: the reader iteratively takes an $x \in X$ and finds a $y \in Y$ in such a way that satisfies $R(x, y)$ until every member of the set $X$ has been paired with a $y \in Y$. That is, the interpreter selects $x_{1} \in X$ and then finds an $y_{1} \in Y$ such that $R\left(x_{1}, y_{1}\right)$, then selects $x_{2} \in X$ and then finds an $y_{2} \in Y$ such that $R\left(x_{2}, y_{2}\right)$, and this process continues for every member of the set $X$. Alternatively, consider the generic EA statement: There exists $y \in Y$ such that for all $x \in X, R(x, y)$. Player $\mathrm{E}$ starts the game by attempting to find a $y^{*} \in Y$ so that no matter what $x \in X$ Player A selects in the following turn, $R\left(x, y^{*}\right)$ will still be satisfied. This is analogous to interpreting an EA statement as follows: the reader attempts to find one $y^{*} \in Y$ such that $R\left(x, y^{*}\right)$ is satisfied for every $x \in X$. The key difference in Player $\mathrm{E}$ winning the game for an EA statement in comparison to an AE statement is that Player E can show the claimed relation is satisfied in one turn. For an AE statement, Player E can take multiple turns (as many as the cardinality of $X$ ) and play a potentially different $y \in$ $Y$ for each of the previously played $x \in X$.

In contrast to the dynamic construals implied by Dubinsky and Yiparki's (2000) game, Dawkins and Roh (2019) offer static each-to-some and one-to-every relationships as normative construals of $\mathrm{AE}$ and EA statements, respectively. I view these relationships as the outcome of the dynamic construal, or to use the game analogy, a snapshot of the potential game moves. These construals focus on the potential dependencies between the first and the second quantified variables. Using the static construals, the true AE 
statement above (i.e. Example 1) conveys that each positive real number $s$ is paired with some point $C$ on the ray $\overrightarrow{A B}$ in such a way that the distance between $A$ and the point $C$ is $s$ (each $s$ to some $C$ ). Whereas the true EA statement above (i.e. Example 3) conveys that there is one real number $M$ that is paired with every real numbers $x$ in such a way that $\sin (x)<M$ (one $M$ to every $x$ ).

Dawkins and Roh (2019) offer diagrams of the pair-wise relationships. Figure 1 offers the four pair-wise relationships that are described by statements constructed by permutating the order of the quantifiers and variables of the statement in Example 1:

AE Statement conveying each $s$ to some $C$ : For every positive real number $s$, there exists a point $C$ on a ray $\overrightarrow{A B}$ such that $d(A, C)=s$.

EA Statement conveying one $C$ to every $s$ : There exists a point $C$ on a ray $\overrightarrow{A B}$ such that for every positive real number $s, d(A, C)=s$.

AE Statement conveying each $C$ to some $s$ : For every point $C$ on the ray, there exists a positive real number $s$ such that $d(A, C)=s$.

EA Statement one $s$ to every $C$ : There exists a positive real number $s$ such that for every point $C$ on the ray, $d(A, C)=s$.

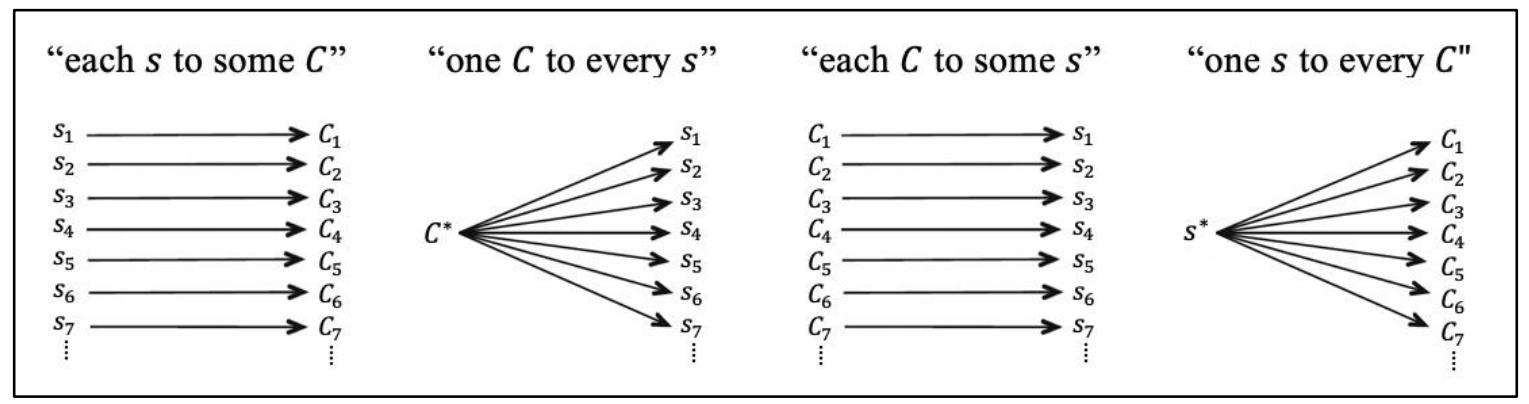

Figure 1. Pairwise relationships (adapted from Dawkins \& Roh, 2019, p. 4). 
Dawkins and Roh (2019) highlight some potential complexities with the pairwise relationships. First, they explain that the diagrams could be an overgeneralization of the relationships between quantified variables in AE statements. They point out that each-tosome relationships do not require every element in the second set be paired with an element from the first set. For instance, consider a defining property for what I called "Prime Sequence": For all prime numbers $p$, there exists a natural number $n$ such that $a_{n}=p$. Using the pair-wise relationships, this conveys that each $p$ is paired with some index $n$ such that $a_{n}=p$; however, not every index value has to be paired with a prime number. For instance, the following is an example of a Prime Sequence:

1, 2, 1, 3, 1, 5, 1, 7 ... (see Figure 2). Dawkins and Roh also say that each-to-some relationships might have multiple elements of the first set paired with the same element in the second set. While one cannot come up with such an example with a Prime Sequence since a sequence must be a function from the set of natural numbers to the set of real numbers, one can construct such an example with another AE statement. Consider the following version of the Archimedean Property of real numbers: For all real numbers $x$, there exists a positive integer $m$ such that $m>x$. One could pair multiple real numbers with the same positive integer (see Figure 2). Yet, another complexity is that members of the first set can be paired with multiple members from the second set. For instance, the following is a Prime Sequence, even though each prime number is paired with two index values: $2,2,3,3,5,5,7,7, \ldots$ (see Figure 2 ). 


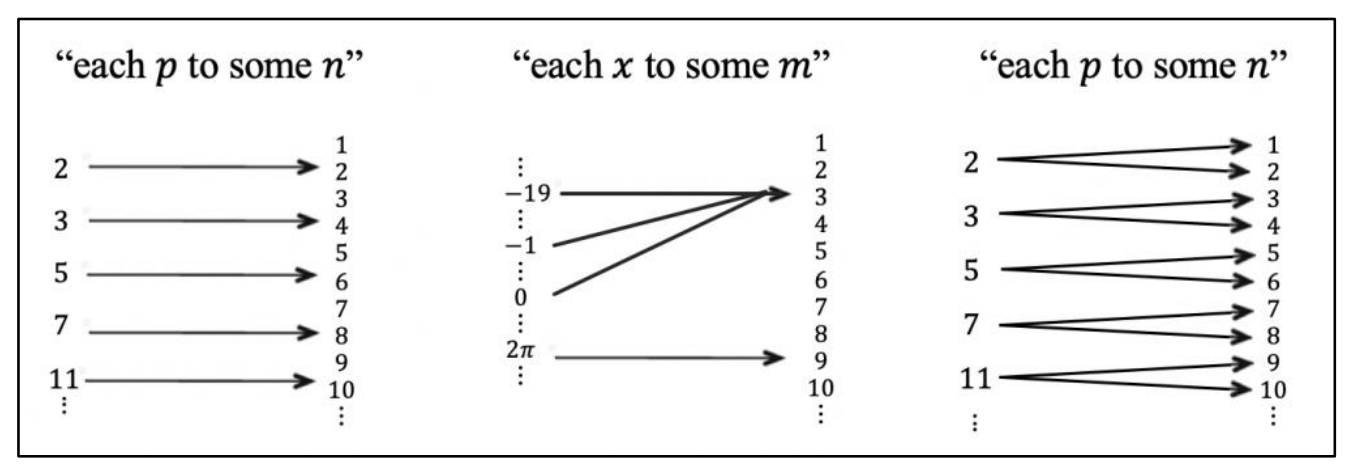

Figure 2. Examples of each-to-some relationships.

Additionally, Dawkins and Roh (2019) point out that the normative construals of any true EA statement and the corresponding AE statement is "rather difficult to distinguish" (p. 21). For an example, consider a statement from their survey in reference to the function $f(x)=\sin (x)$ :

(True) EA Statement: There exists a real number $M$ such that for all real numbers $x$, $f(x)<M$.

(True) AE Statement: For all real numbers $x$, there exists a real number $M$ such that $f(x)<M$.

Since $f(x)=\sin (x)$ is bounded (i.e. the EA statement is true), a single value of $M$ (e.g. $M=2$ ) satisfies the relationship $f(x)<M$ for all real numbers $x$. This can be viewed as both (a) one $M$ to every $x$ since $M=2$ is greater than $\sin (x)$ for every real number $x$, and (b) each $x$ to some $M$ since each real number $x$ is paired with some real number (and that real number happens to always be 2). Because of this complexity, the authors had trouble distinguishing students' interpretations of the above statement as describing either a one-to-every or an each-to-some relationship. The dynamic construals help explain this phenomenon. Suppose the following generic EA statement is true: There 
exists $y \in Y$ such that for all $x \in X, R(x, y)$. Since it is true, Player E can find one $y^{*} \in Y$ such that $R\left(x, y^{*}\right)$ is satisfied for every $x \in X$. That also means that if they were to play the game with the corresponding AE statement then Player E could always select $y^{*} \in Y$ during their turn (i.e. $\left.\left(x_{1}, y^{*}\right),\left(x_{2}, y^{*}\right), \ldots\left(x_{n}, y^{*}\right), \ldots\right)$.

Dawkins and Roh's (2019) construals defined by each-to-some and one-to-every relationships are quite different than the ones suggested by Dubinsky and Yiparaki's (2000) game. Dawkins and Roh's (2019) describe static pair-wise relationships between the universally quantified variable and the existentially quantified variable. In contrast, Dubinsky and Yiparaki's (2000) game suggests that the normative construals of AE and EA statements are dynamic processes that coordinates selecting $x \in X$, selecting $y \in Y$, and evaluating $R(x, y)$. I view the pair-wise relationships as a snapshot of the dynamical process result. When working with the students in my study I found that the pair-wise relationships were insufficient for studying the complex ways in which my students were making sense of the statements. This is because one-to-every and each-to-some relationships that can be detected from the order of the quantifiers can generalize EA and $\mathrm{AE}$ Processes in AE or EA statements but they do not necessarily describe the exact relationships described in a particular statement. In my study, the students were thinking more systemically about the processes that are encoded in the statement. This suggests that there is more to consider than the order of the quantified variables to understand normative construals of AE and EA statements. In this study, I consider the normative construals of the EA and AE statements to be the dynamic construals analogous to Dubinsky and Yiparaki’s (2000) game. 


\section{Students' interpretations of statements with quantifiers}

The existing research literature on student's interpretations of statements with multiple quantifiers has identified aspects that affect students' construals of such statements, including semantics, pragmatics, and syntax. Semantics refers to the reader's understanding of the ideas referenced in a statement and pragmatics refers to the plausibility or relevance of an interpretation (Dawkins \& Roh, 2019). The syntax refers to "the arrangement of and relationships among words, phrases, and clauses forming sentences" (Dubinsky \& Yiparaki, 2000, p. 1). The existing research on students' understanding of the syntax of statements with quantifiers has mainly focused on the order of the quantifiers and the relationships among the quantified variables.

The literature has pointed to students' lack of attention to and understanding of the order of quantified variables (Dawkins \& Roh, 2019; Dubinsky \& Yiparaki, 2000; Durand-Guerrier \& Arsac, 2005; Roh, 2010; Roh \& Lee, 2011). Dubinsky and Yiparki (2000) surveyed and interviewed students about their interpretation of EA and AE statements. The set of statements included statements in the natural context (e.g. There is a mother for all children) and two statements in the mathematical context (e.g. For every positive number $a$ there exists a positive number $b$ such that $b<a$ ). They found that novice students tended to interpret EA statements as AE statements. They also reported that students rarely considered a different interpretation even after significant probing (e.g. asking them directly if a statement could be interpreted differently) and that many students in their study were unaware of their own interpretation process. Dubinsky and Yiparki suggested that semantics played the dominant role in students' interpretations of 
statements with multiple quantifiers, explaining that the way students interpreted statements was based on the way they viewed the world. For instance, the students in their study were likely to interpret 'there is a mother for all children' as 'every child has a mother' since that fits more with their understanding of the world than 'there is one mother that is the mother of every child'.

One possible explanation that Dubinsky and Yiparaki (2000) explored that is particularly relevant to the current study is whether the use of 'all' versus 'every' impacted the way that students interpreted the statements. They posited this as a reasonable conjecture since "the word 'all' seems to be collective" and that "the word 'every' appears to address each case individually” (p. 22). They hypothesized that if students made this distinction then they would likely interpret statements with 'all' as an EA statement whereas statements with 'every' would be interpreted as an AE statement. However, they argued that this was not supported by their data. They found that $81 \%$ of the students $(\mathrm{N}=63)$ interpreted 'there is a mother for all children' as an AE statement whereas only about $40 \%$ interpreted 'there is a perfect gift for every child' as an AE statement (emphasis added). They also tested whether the use of 'every' would be interpreted as an AE statement by comparing students' interpretations to 'someone is kind and considerate to everyone' and 'there is a magic key that unlocks everyone's heart' (emphasis added). They reported $11 \%$ of the students interpreted the first statement as an AE statement and $37 \%$ of the students interpreted the second as an AE statement. It is important to note here that they did not test this theory with the statement in the mathematics context (both statements used 'every'). It is plausible that students might 
construe different meanings with mathematical statements that use 'all' versus 'every' since the mathematical register (Halliday, 1978) is more technical than the natural context.

Dawkins and Roh (2019) further investigated influences on students' interpretations with a survey. The survey featured eight different mathematical statements with multiple quantifiers from different content domains. They administered the survey to students before and after their Introduction to Proofs class and analyzed survey responses from students who completed both the pre- and post- test (a total of 77 students). They reported the mathematical context had more of an influence on students' interpretations than the order of the quantifiers and that students became more sensitive to the order of the quantifiers after they completed the course. They also found that students' attention to pragmatics influenced their interpretations. The students were less likely to describe the appropriate one-to-every or each-to-some relationship when the statements were less interesting or relatively absurd than those that more interesting or relevant. For instance, the following statement proved challenging for the students in their study: there exists a point $C$ on a ray $\overrightarrow{A B}$ such that for every positive real number $s, d(A, C)=s$. Dawkins and Roh rationalized that the statement was blatantly false; it's relatively absurd to claim the distance between two points on a ray is every positive real number.

\section{Toward a functional approach of statements with multiple quantifiers}

Halliday's social semiotic theory of language, known as Systemic Functional Linguistics (SFL), deals with the construction and representation of meaning and is built on the assumption that "language is not realized in the abstract; it is realized as the 
activity of people in situations" (Halliday, 2009, p. 18). That is, members of a (sub-) culture must have some sense of shared contextual meaning in order to communicate (O'Halloran, 2008). Systemic Functional Grammar (SFG) is part of SFL (Halliday \& Matthiessen, 2013). Hallidayan scholars studying the grammar of scientific language, including mathematics (e.g. Lemke, 2003; O'Halloran, 2008, 2015), adopt Halliday's view that the difficulty with using and interpreting scientific language "lies more with the grammar than with the vocabulary" (1996, p. 78). Halliday elaborates on this notion:

"The problems with technical terminology usually arise not from the technical terms themselves but from the complex relationships they have with one another. Technical terms cannot be defined in isolation; each one has to be understood as part of a larger framework, and each one is defined by reference to all the others" (1996, p. 78).

To learn mathematical language, one must learn how to use the vocabulary - the patterns associated with the terms, in order to construct mathematical concepts.

Halliday's notion of grammar is "functional" and in contrast to traditional school grammar. Gebhard and Martin (2011) comment that traditional school grammar consists of the study of parts of speech and prescriptive rules for correct usage. By contrast, Halliday's conceptualization of grammar emphasizes the role that something plays in the structure - its function. The 'systemic' in SFG highlights that language is comprehensive in the sense that "what [is] said about any one aspect also contributes to the total picture" (Halliday \& Matthiessen, 2013, p. 20). Halliday and Matthiessen explain that "when we [analyze] a text, we show the functional organization of its structure; and we show what meaningful choices have been made" (p. 24). In this way, I view the construction of the dynamic construal of $\mathrm{AE}$ and $\mathrm{EA}$ statements as a functional approach toward understanding the grammar of such statements. 
My investigation of the students' thinking about the grammar of statements with quantifiers was guided by Halliday's theory. In particular, I studied the students' considerations of the grammatical choices and their function in conveying meaning. Both the system (paradigmatic ordering in language) and the structure (syntagmatic ordering in language) of mathematical statements are important to the grammar. The system answers "what could go instead of what" (Halliday \& Matthiessen, 2013, p. 22); whereas, the structure is the patterns or regularities in language, answering "what goes together with what” (p. 22). Gebhard and Martin (2011) offered an example: 'Al wrote an essay'. Alternatively, one could say 'Al has written an essay' since 'has written' could go instead of 'wrote'. Additionally, 'will write' could go instead of 'wrote', to convey that Al's writing will happen in the future instead of it happening in the past. Another choice is that one could use 'has Al' to go with 'written an essay' to formulate a question of whether Al has written an essay instead of declaring it. Halliday and Matthiessen describe the systems and the structure as working together, explaining "each system — each moment of choice - contributes to the formation of the structure" (2004, p. 24). To determine the significance a particular word or phrase's function, semioticians use what's called a communication test and select a word or phrase and then consider alternatives (Chandler, 2004). The test is used to understand the function and identify what Bateson (1979) refers to as 'differences that make a difference'. For instance, changing 'Al has' to 'has Al' makes a difference to the construal of 'Al has written an essay' - the declaration changes to a question. In my study, I leveraged communication tests to 
investigate differences in grammar that make a difference to the students' construal of the mathematical statement.

\section{Present Study}

The present exploratory study aims to investigate students' construals of mathematical statements with multiple quantifiers. I answer: What grammatical complexities do students consider when constructing and interpreting mathematical statements with multiple quantifiers? It is important to note that while I present differences in novice and expert uses of the language it is not meant to be interpreted as identifying students' misconceptions of mathematical language. Rather, here I argue that students can come up with creative ways to use language to express their thinking and in doing so their thinking gives insight about the complexities of the standard use of the language.

\section{Methods}

This study is part of a larger design research project that aims to enhance a local instructional theory in real analysis to accommodate the goal of supporting students with proof related activity. Here, I present a retrospective analysis of data from a total of four undergraduate students over the course of two rounds of design experiments (Cobb \& Gravemeijer, 2008) in a laboratory setting. I refer to the two students in the first experiment as Chloe and Gabe and the students in the second experiment as Ada and Lori.

The primary goal of the first design experiment was to support students in learning about foundational concepts in real analysis by engaging them in an inquiry- 
oriented task sequence (for more information about this task sequence see Strand, 2016). While working with Chloe and Gabe, it was evident that they were not only learning about real analysis concepts but they also seemed to learn about mathematical language and proving. Ada and Lori were recruited from an Introductions to Proof class at a community college where the larger project team began to scale up the instructional sequence in real analysis to a whole classroom setting. Thus, Ada and Lori engaged with some of the refined tasks from the experiment with Chloe and Gabe prior to participating in my second laboratory design experiment. The primary goal of the experiment with Ada and Lori was to support them in learning about mathematical language and using definitions in proofs.

Chloe and Gabe mostly earned A's in their mathematics course work. At the time of the first experiment, both students had taken most of the introductory mathematics courses for STEM majors at their university, including Linear Algebra. Neither student had taken an advanced mathematics class characterized by proof. Ada and Lori also typically excelled in their mathematics classes. Prior to the Introduction to Proofs course, Ada had completed the single-variable calculus courses and Lori had completed all introductory mathematics courses offered by the community college (i.e. the full calculus sequence, Differential Equations, Linear Algebra).

I met with Chloe and Gabe for a total of 10 sessions and with Ada and Lori for 11 sessions. Each teaching session lasted approximately 1.5 hours. All sessions were recorded on multiple devices (audio and video) and at least one witness was present. I shared the teacher-researcher role with another researcher during the first design 
experiment and then was the primary teacher-researcher during the second. The project team debriefed between each session, reviewing our interpretations of what happened and revising our plan for the following session.

Realistic Mathematics Education (RME) guided the overarching instructional approach. Driven by principles of guided reinvention, we were committed to supporting students to construct mathematics from their informal mathematical ideas (Gravemeijer, 1998). Throughout the experiments, students were engaged in writing mathematical statements (writing defining properties or conjecturing) to articulate their thinking. The students were encouraged to write down their idea (often times informally) and then the students and teacher-researcher would work to refine the statement so that it was more formal and more accurately articulated the idea. This type of data is quite different than the data analyzed in prior studies on students' understandings of statements with multiple quantifiers. Prior studies have investigated students extracting meaning from a given statement (Dawkins \& Roh, 2019; Dubinsky \& Yiparki, 2000), whereas this study explores students attempting to articulate their own meaning by writing a statement. My approach gave me a unique window into how the students connected the grammatical structures and word choices to their mathematical meanings.

The retrospective analysis for this study began with watching the video of each of the teaching sessions and creating content logs of each of the design experiments. The content logs were detailed chronological descriptions of the tasks and the activity that occurred in each session. Often times the content logs included transcribed excerpts or pictures of student work. I reread all of the content logs in order to identify students' non- 
normative construals and then re-watched corresponding video of such instances. During this pass of the data, I tagged instances when students exhibited unconventional ways of using or thinking about mathematical language when writing or interpreting statements. In particular, I considered each written statement that was produced by a student, asking myself: Are there unconventional ways in which students used mathematical language? My assessment relied on both my mathematical knowledge as well as research literature on students' use of mathematical language (e.g. Dawkins \& Roh, 2019; Lew \& MejíaRamos, 2015). When I identified statements that were unconventional, I described what I perceived to be unconventional and made note of insight I had about the students' thinking. Grammatical complexities with the universal quantifiers, existential quantifiers, and 'where' (or other equivalent phrases like 'such that') in statements with multiple quantifiers emerged from reviewing my responses to these guiding questions.

I then returned to the content logs and corresponding video to further explore when students discussed the grammatical complexities with the universal quantifiers, existential quantifiers, and 'where'. During this pass of the data, I identified statements that provided a communication test of the students' thinking regarding the grammar of universal quantifiers, existential quantifiers, and 'where'. In particular, I search for instances when the students considered alternatives with a selected universal quantifier, existential quantifier, or phrases like 'where' and discussed the conveyed meaning. By alternatives, I mean both alternative choices in phrases (e.g., 'for all' instead of 'for any') and alternate structural choices (e.g., 'for all... there exists... where' instead of 'there exists... for all... where...'). To analyze the students' thinking about these statements, I 
aimed to answer how the students might answer 'what could go instead of what' (and why) or 'what goes together with what' (and why) based on their discussion in comparison to my understanding of the standard conventions. I then returned to additional episodes that featured the grammatical complexities and aimed to make sense of the students' use or interpretation of statements given my hypothesized student answers to the guiding questions. This paper details my findings from exploring the grammatical complexities with the universal quantifiers, existential quantifiers, and 'where' in statements with multiple quantifiers. In what follows, I will discuss the differences that made a difference for the students.

\section{Results}

In the following section I present instances that exemplify grammatical complexities that the students in my study considered as they constructed and interpreted mathematical statements with multiple quantifiers. I highlight two points: (1) grammatical complexities influenced the students' construals of mathematical statements with quantifiers, and (2) these complexities revealed limitations for the ways that prior research has conceptualized statements with multiple quantifiers. In what follows, I present my findings organized by the students' thinking about the grammar of universal quantifiers, existential quantifiers, and 'where' (or 'such that').

\section{Grammar of universal quantifiers}

In what follows, I show how the students in my study considered different meanings for 'for all', 'for any' and 'for every,' and particularly how students construed a collective meaning of 'for all', which led the students to construe non-normative 
meanings for mathematical statements. Then, I present an example of a statement that students constructed with the collective meaning of 'for all'.

Students might see differences in 'for all,' 'for any,' and 'for every'. Ada and Lori discussed the differences they saw between 'for all', 'for any', and 'for every'. Ada explained, 'I feel like 'for any' sounds more singular, and I think 'for all' sounds a little bit more plural" (and later explained that, to her, 'for every' also sounded singular). By singular, Ada meant "you can just pick anything," meaning you can consider one, arbitrary member of the set. On the other hand, Ada imagined selecting the whole set that the quantified variable belonged to (e.g. $X$ ) at once for the phrase 'for all $x$ in $X$ '. This is consistent with English grammar, which she used when she wrote these types of phrases more informally. Consider the two phrases in Figure 3; the word 'number' is singular when quantified by 'for every' (Figure 3A) and plural when quantified by 'for all' (Figure 3B). This distinction is similar to Dubinsky and Yiparaki's (2000) hypothesized interpretation that word 'all' construes a collective meaning whereas the word 'every' seems address each case individually.

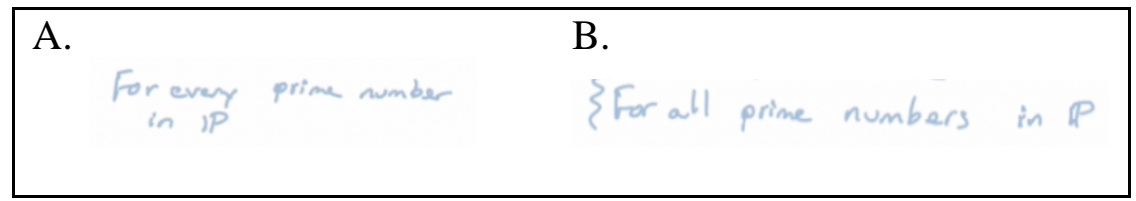

Figure 3. 'number' vs. 'numbers'

Students might construe different meanings with statements that include 'for all' instead of 'for any' or 'for every'. I found that the collective meaning of 'for all' made a difference in the conveyed meaning of a statement with the phrase. Ada and Lori defined what we called a "Prime Sequence" during the ninth session of the second experiment. They understood a prime sequence to be a sequence that contained all the 
prime numbers. Note that during their defining activity they considered using the phrase 'There exists at least one input $k$ for all prime numbers in $\mathbb{P}$ ' where $\mathbb{P}$ stood for the set of prime numbers. They were not satisfied with the phrase because they thought it suggested that one step in the sequence was associated with every prime number and drew the sketch given in Figure 4.

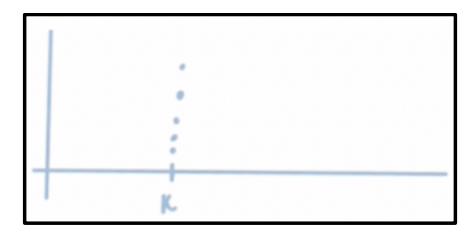

Figure 4. Problem with Ada and Lori's proposed definition.

At the end of the ninth session they were satisfied with their definition (see Figure 5A): A sequence $\left\{a_{k}\right\}$ is prime if for every prime number $p$ in $\mathbb{P}$, there exist at least one input $k$ where $a_{k}=p$. During the next session, I asked Ada and Lori if we could replace the phrase 'every' with 'all':

Lori: No, it has to be a 'for every' doesn't it?

Teacher-researcher: How so? Or why does it have to be 'for every'?

Ada: Because $[\ldots]$ it still sounds like they all go to one $k$-value. They all map to one $k$-value. So, all the prime numbers are like, all the prime number are sitting on top of each other at just one $k$-value.

Teacher-researcher: This does? (pointing to 'for all $p$ in $\mathbb{P}$ ' Figure 5A.)

Ada: Yeah.

Teacher-researcher: This 'for every' or 'for all'?

Ada: If you were to say 'for all'. This is what 'for all' does for number seven (sketches picture in Figure 5B).

Teacher-researcher: Can you explain how it is that?

Ada: Because... [....] So it's like for all of them. They all - it's just they all mapped to one. That's what it sounds like. How do I describe that?

Lori: 'For every' sounds like a little bit more like you're singling out...

Ada: Each individual.

Lori: Here's one [prime number], here's a $k$. Here's one [prime number], here's a $k$. But if you say 'for all' (makes a gesture like she is picking up a large object)... 
Ada: Yeah, exactly! (Laughs)

Lori: And here is a $k$.

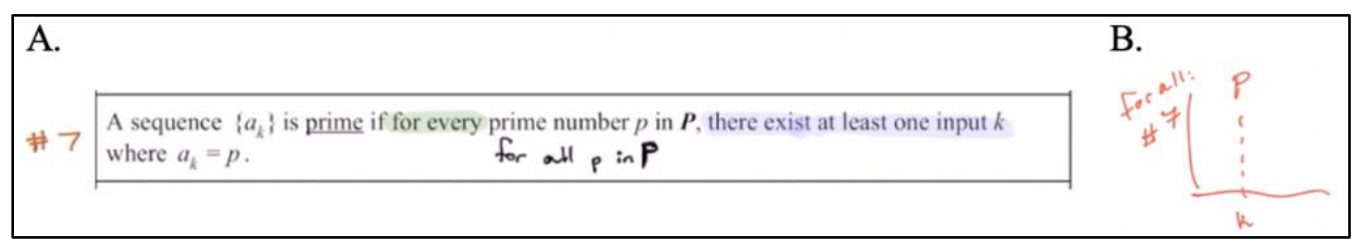

Figure 5. Prime Sequence definition with 'for every' vs. 'for all'

During the above exchange, Lori and Ada saw a problem with replacing 'every' with 'all' in their prime sequence definition. With 'for all $p$ in $\mathbb{P}$, there exist[s] at least one input $k$ where $a_{k}=p$, Ada and Lori first imagined the whole set of prime numbers with the phrase 'for all $p$ in $\mathbb{P}^{\prime}$ (illustrated by Lori's gesture of picking up a large object) and then found one corresponding input ("And here is a $k$ "). The collective construal of 'for all' changed the rules of the game. For Ada and Lori, this change allowed Player A to select all of the prime numbers at once and then Player E was tasked to find an input $k$ such that $a_{k}=p$ for each the prime number $p$ in play. Notice this is essentially the same game that Player E would play using the normative construal of the following: There exists an input $k$ such that for all $p$ in $\mathbb{P}, a_{k}=p$. Of course, this was not what Ada and Lori intended to convey. Instead, they wanted the interpreter to iteratively pair a prime number $p$ with an input $k$ in such a way that $a_{k}=p$. ("Here's one [prime number], here's a $k$. Here's one [prime number], here's a $k . ”)$

Chloe and Gabe also seemed to see a distinction between universal quantifier phrases. The students were tasked to negate one of their conjectures that was equivalent to the Archimedean Property (see Figure 6A). Specifically, the teacher-researcher asked, "What would have to happen if it wasn't true?" Chloe offered the statement given in 
Figure 6B. Notice the similarities in the two statements; Chloe essentially switched the real number $x$ and the positive number $m$ and then negated the inequality so that it said $x \geq m$ instead of $m>x$. Chloe explained, "So that says for any positive integer there is a real number where the real number is larger than the integer. (long pause) Or the real number is larger than all the positive integers." After the teacher-researcher prompted them to reconsider what Chloe wrote, they had the following interaction:

Gabe: I think that for any positive integer, yeah there would exists a real number that is larger or equal to it, right?

Chloe: Yeah, I know, but the $x$ is one number. And so, we are saying for any number, like all the positive integers are less than it.

Teacher-researcher: So the question is - is what you're saying now the same thing as what you wrote?

Chloe: Ehhh - kind of.

Gabe: I think that if it is 'for all' positive integers.

Chloe: Yeah! Yeah. (Chloe changes it to say 'for all' instead of 'for any', see Figure 6C)

The following is my interpretation of the exchange: Gabe first suggested that

Chloe's proposed statement (Figure 6B) seemed true but the statement was supposed to be false. Then, Chloe explained that it said (or at least she wanted it to say that) there was one real number $x$ such that $x \geq m$ for each positive integer $m$ ("but the $x$ is one number [...] like all the positive integers are less than it"). When they were asked if that's what the statement actually said, Gabe suggested changing the word 'any' to 'all,' suggesting a change from imagining one arbitrary positive integer to the whole set of positive integers. Chloe agreed with the alteration suggesting that the alteration represented her intended meaning.

Chloe then continued to explain the statement in Figure 6C: 
"You're not choosing multiple $x$ 's, you're choosing one $x$, you're choosing like 100 is $x$ and then you're saying that all the possible positive integers are less than 100 . We're not going around like oh there is 101 so our $x$ is now 1000. [That] is the point that I am trying to say."

Chloe's response suggests that to her the 'for all' version of the statement conveyed that there was one $x^{*}$ in the set of real numbers such that $x^{*} \geq m$ is satisfied for every positive integer $m$. Notice that this fits the dynamic construal of the following statement: There exists a real number $x$ such that for all positive integers $m, x \geq m$. That is, the statement instructs the interpreter to find a one real number $x^{*}$ (e.g. $\left.x^{*}=100\right)$ so that $x^{*} \geq m$ is satisfied for every positive integer $m$.

\begin{tabular}{|lll|}
\hline A. & B. & C. \\
"for any real number $x$ & "for any pos int $m$ & "for all pos int $m$ \\
there exists a pos int $m$ & there exists a real num & there exists a real num \\
such that $m>x "$ & $x$ where $x \geq m$ " & $x$ where $x \geq m$ " \\
\hline
\end{tabular}

Figure 6. Using 'for all' to construe one-to-all meaning.

Both pairs of students illustrated that students might answer the questions of "what could go instead of "for all'?" differently than the larger mathematics community. In particular, the mathematics community takes 'for all' and 'for any' as equivalent phrases (Batty \& Woodhouse, 1994) used to refer to each member of the set that the universally quantified variable belongs to. Alternatively, the students considered a collective meaning of 'for all'. The collective meaning essentially changes the meaning of an AE statement to the normative construal of an EA statement. In particular, if students use the collective meaning then 'for all $x$ in $X$, there exists $y$ in $Y$ such that 
$R(x, y)^{\prime}$ ' is likely to convey that there is one $y^{*} \in Y$ such that $R\left(x, y^{*}\right)$ is satisfied for every $x \in X$.

Students creatively use the collective meaning of 'for all'. The example comes from earlier in the ninth session with Ada and Lori when they were defining Prime Sequence. At this point in the session, the students had created examples and nonexamples of prime sequences and offered an informal definition ('A prime sequence is a sequence that must contain the set of prime numbers in its range.'), but had not yet written the statement discussed earlier in the section (Figure 5). As an attempt to support Ada and Lori in refining their informal definition, I requested that they rewrite their statement using 'for all' and/or 'there exists'. Notice that this is a quite a challenging task if one were to use the collective meaning of 'for all'. Conventionally, one might offer the following: for all $p \in \mathbb{P}$, there exists $k \in \mathbb{N}$ such that $a_{k}=p$. Of course, this would not be an option for someone using the collective meaning of 'for all' since they would possibly construe the following with the statement: there is one $k^{*} \in \mathbb{N}$ such that $a_{k^{*}}=p$ for every prime number $p$.

After my request, Ada quickly responded "I definitely [want to] put the 'for all' with the prime numbers like - for all prime numbers". She expressed that she wanted their statement to convey that, "each prime number has its own natural number." Then, Ada and Lori produced statement Figure 7. I suggest the following as Ada and Lori's construal of their statement: There is step in the sequence that lists a particular prime number and this is the case for the set of prime numbers. 


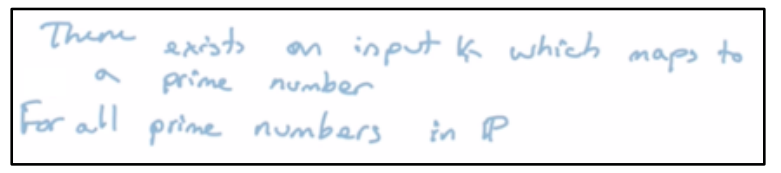

Figure 7. Proposed property for Prime Sequence definition.

This statement illustrates how students might craft a statement with the collective meaning of 'for all' in order to communicate that the reader can iteratively find an input $k$ for every prime number $p$. Essentially, what goes together with the collective meaning of 'for all'? In this case, 'there exists an input $k$ which maps to a prime number' functioned as a way to find an input to for a prime number and 'for all prime numbers in $\mathbb{P}^{\prime}$ functioned to iteratively find pairs for every member of the set of prime numbers. The statement is quite unconventional and a creative way to define prime sequence using the collective meaning of 'for all'.

\section{Grammar of existential quantifiers}

In the following section I will discuss the differences that students considered between variants of existential quantifiers. In particular, students construed two different meanings for 'there exists': (1) there exists a unique value and (2) there exists at least one value. These differences led students to non-normative construals of statements with existential quantifiers. Then, I discuss an example that highlights that students might think of a 'key one' when they use 'there exists'.

Students might see differences in 'there exists at least one' and 'there exists' and construe different meanings from statements with these phrases. Recall Ada and Lori's definition for Prime Sequence ("A sequence $\left\{a_{k}\right\}$ is prime if for every prime number $p$ in $\mathbb{P}$, there exists at least one input $k$ where $a_{k}=p . "$, see Figure 5A). Later in 
the ninth session with Ada and Lori, I aimed to determine the significance of 'at least one' in their definition and asked if they could simply use 'there exists an' instead. Ada was hesitant, explaining, "Well it doesn't matter too much. But it also kind of insinuates that each prime number can't be repeated that was my only concern" (emphasis added). I take this to mean that Ada was concerned that without the phrase 'at least one', a reader could interpret their statement to mean that each prime number was paired with exactly one input. This was unsettling for Ada since they previously agreed that a prime sequence could list a prime number more than once. For instance, $\{2,2,3,3,5,5,7,7, \ldots\}$ was still a Prime Sequence even though 2 was paired with $k=1$ and $k=2$. However, the normative construal of 'there exists' is 'there exists at least one'.

Ada and Lori expressed a similar concern during the tenth session when I asked them to interpret the following:

A function, $f: X \rightarrow Y$, is type-2 if for all $y$ in $Y$ there exists a $x$ in $X$ such that $y=f(x)$.

Note that conventionally one would construe the concept of an onto function with the above definition. However, Ada and Lori considered different interpretations than one would expect. Their non-normative can be explained by applying an 'exactly one' construal of 'there exists' and then later applying a collective construal of 'for all'. When I initially presented this definition to the students, they took several minutes to think privately about the given definition. In the discussion that followed, they described several different interpretations that they were considering. Note that they did not seem convinced that one interpretation was the "correct" interpretation, nor did they necessarily agree with each other's interpretations. In what follows, I will describe two of 
their interpretations related to the 'exactly one' construal of 'there exists' starting with Ada describing one (non-standard) interpretation that she was considering. Her interpretation of the definition was essentially that all type- 2 functions are one-to-one functions, which could be explained by 'exactly one' construal of 'there exists'. Then, I will describe another interpretation that Lori introduced, which suggested that she was considering a type-2 "function" as not being well-defined. Lori's interpretation can be explained by using the 'exactly one' construal of 'there exists' and the collective construal of 'for all'.

When I followed up with Ada about her thinking, she pointed to a parabola that she previously drew (see Figure 8 below). She explained that it was not type 2 because " $y$ maps to two different values on both sides" and then continued to explain her interpretation of the statement, "it says 'there exists a $x$ in $X$ ' (emphasizing 'a') it sounds like there is only one $x$ for each $y$ ". Similar to the previous example, Ada's construal of 'there exists a $x$ in $X$ ' was 'there is exactly one $x$ in $X$ '. Ada's interpretation of the statement seemed to suggest that each $y$ in $Y$ must be paired with a unique $x$ in $X$ so that that $y=f(x)$. To Ada, in order for a function to be type-2, it must be one-to-one.

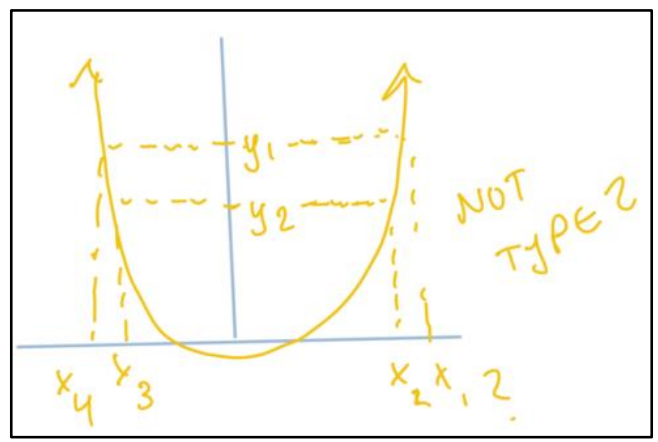

Figure 8. Ada's non-example of type 2 function. 
Lori's interpretation also considered the 'exactly one' construal of 'there exists' and then she also considered the collective construal of 'for all'. In particular, when I followed up with Lori about Ada's interpretation she Lori echoed the 'exactly one' construal, explaining 'it all comes down to that 'there exists a $x$ ' (emphasizing 'a'). Because that could mean that there exists a unique $x$ in $X^{\prime}$. Here, Lori revoiced Ada's interpretation that 'there exists a $x$ in $X$ ' could be interpreted as 'there is exactly one $x$ in $X$ '. However, then she considered the collective construal of 'for all' which led to a different interpretation of the statement than the interpretation that Ada was considering. In particular, Lori suggested that the statement claimed they could find one unique $x \in X$ so that $f(x)=y$ for every $y \in Y$. She explained, "and that would mean it would be that guy (pointing to the Figure 9 below) because that means one $x$ works where $f(x)$ equals all $Y . "$ And then continued to explain, "because you have one $x$-value, one unique $x$ in big $X$ that maps to all $y$." Notice that the collective construal of 'for all' influenced Lori's interpretation so that type-2 "functions" were not well-defined. Additionally, the "exactly one' construal of 'there exists' further influenced her interpretation by making type-2 "functions" as extreme cases of not well-defined mappings, and particularly, a map that assigned a single input to every value in $Y$. Lori's interpretation seems to suggest a drastically different game. In particular, 'for all' allows Player A to select all of $y$ in $Y$ at once and then Player E must find exactly one $x$ in $X$ so that that $y=f(x)$. After Lori presented the sketch in Figure 9 and her rationale, Ada immediately refuted this interpretation, and saying, "but it's not a function!” Then, Ada returned to her previous 
interpretation (seemingly forgoing the collective meaning of 'for all'), and said "every $y$ maps to any - its own $x$ in the function".

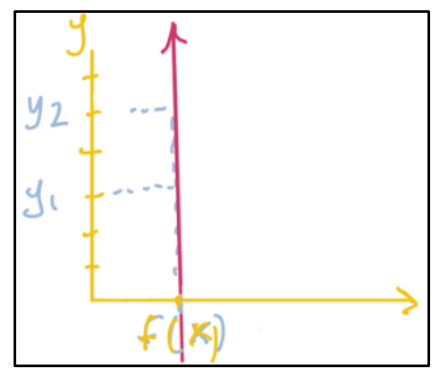

Figure 9. Lori's construal of type-2 definition.

In the previous examples, Ada and Lori demonstrated that they might answer what could go instead of 'there exists at least one' differently than the mathematics community. The mathematics community would answer the question with 'there exists' (or 'there exists a'). However, the data presented here suggests that Ada and Lori would not allow 'there exists a' to replace 'there exists at least one' since 'there exists a' suggests uniqueness. This highlights a difference in the conventional word choice and Ada and Lori's word choice. Instead of taking 'exactly one' to be the conventional meaning of 'there exists,' the mathematics community uses 'at least' to be the conventional meaning. When uniqueness is important, the mathematics community adds 'unique' or 'exactly one' to 'there exists' (Alcock, 2012; Batty \& Woodhouse, 1994). Alternatively, Ada and Lori highlighted a different choice. Instead of using the 'at least' meaning of 'there exists' they used the 'unique' meaning. Ada and Lori used 'at least one' when more than one was important (e.g. more than one prime number in the Prime definition). 
A student's use of 'these exists at least one' in place of 'there exists' is not likely to cause confusion to an interpreter from the larger mathematics community since these phrases are equivalent to the mathematics community. However, if a student with the 'exactly one' construal of 'there exists' reads a statement with 'there exists' rather than 'there exists at least one' then, like Ada and Lori, they might construe an unintended meaning. Additionally, using the 'exactly one' construal of 'there exists' can be further complicated with another non-normative construal. In the example above, Lori used the collective construal of 'for all' and the 'exactly one' construal of 'there exists,' which called her to consider the non-normative construal "one unique $x$ in big $X$ that maps to all $y$ ". This is a drastically different interpretation than the intended meaning.

\section{Students may imagine a 'key one' when they use 'there exists'. The next} example comes from the third session with Ada and Lori when they defined "Eventually Constant Sequence." See Figure 10. They intended the definition to describe a sequence that had an index value such that every index value after it mapped to the same real number. Notice that their definition used 'there exists some natural number $L$ '. For a full description of the defining activity see Chapter 3.

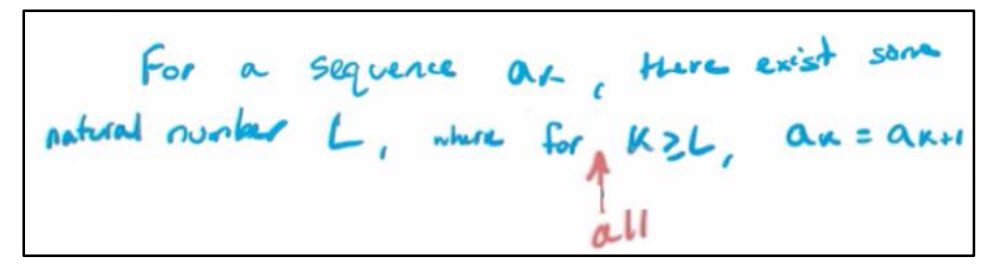

Figure 10. Eventually Constant Sequence definition

After they constructed their definition, I asked them to explain how their definition was satisfied by a sequence that they previously presented as an example of Eventually Constant Sequence (see Figure 11A). 
Ada: I notice that there are a whole bunch of terms over here and they look constant to me so I worked backwards to the beginning. So, if they are all equal to the same value like $y=7$, and we would be like 'how far back does it equal 7?' Well like this one equals 6 so that one isn't going to work, like $y=6$ here. But this one equals 7 and it is the first of this groups of $7 \mathrm{~s}$ and so I would say this one is $L$. And so that means that for all $k$ beyond that $L$ it should be constant and it is. (See Figure 11B)

$[\ldots]$

Teacher-researcher: What if I picked my $L$-value... (labels another $L$ on the horizonal axis, see Figure 11C)

Ada: Why would you do that?

Teacher-researcher: Well would that be ok?

Ada: It wouldn't because - oh this is awesome - you're like totally picking at this (looks at the definition). Wow! It would work by our definition so we need to fix that!

Lori: Do we?

Ada: I think we need to fix it. There exists some natural number... because for all $k$ above this random $L$ that she chose it is all equal to each other so we can totally say that's $L$. So how do we fix that?

Lori: Does it matter though? Isn't the $L$ just arbitrary to show... because it is infinitely long.

Ada: It is arbitrary, I can understand that but I think that if we are going to use this $L$ definition, I think that $L$ should be a very specific thing. And I think our original definition was that $L$ was the very first of the constant - the section where it appears constant. Does that make sense?

Lori: Yeah, I just don't know if it is necessary.

Ada: It doesn't sound necessary, I know what you're saying. But I feel like there is something that is telling me that it is necessary.

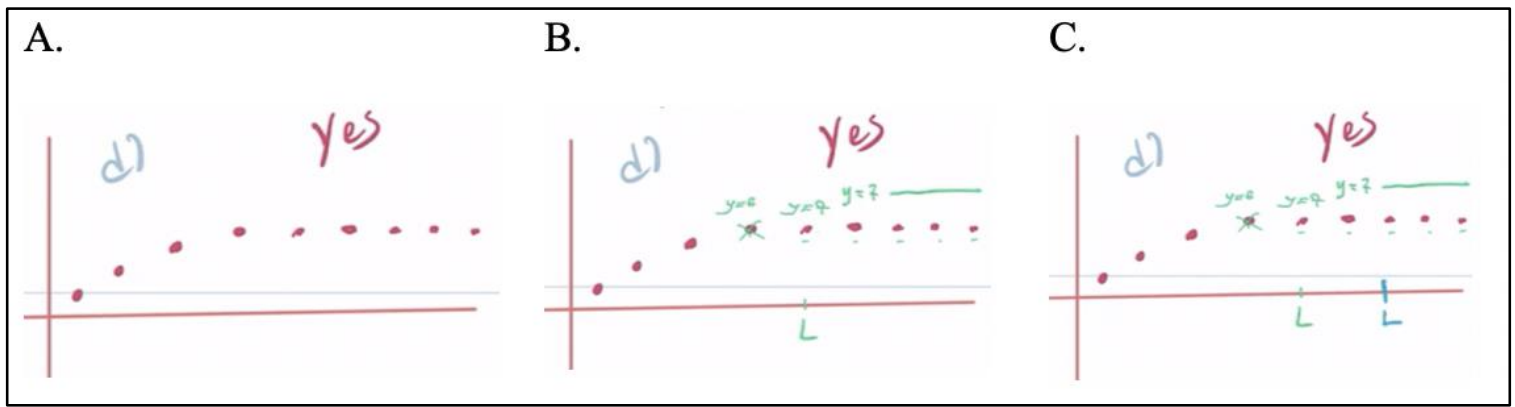

Figure 11. Potential L-value. 
In this example, Ada explained that she intended 'there exists some natural number $L$ ' to be one particular index value, and specifically, the first index value such that every index value after it maps to the same real number. However, the students realized that their definition allowed one to pick a $L$ that wasn't the particular $L$ that the students were considering. Ada was alarmed that this was not captured by their definition and expressed a desire to alter it ("Wow! It would work by our definition so we need to fix that!"). However, Lori questioned if this was a needed change, suggesting that the concept could be defined without identifying the first $L$.

The students then considered their collection of examples and non-examples of Eventually Constant Sequences that they previously created to see if there was an issue with any of their (non-)examples from choosing an $L$ greater than the first index value that satisfied the condition. Ada said, "I am not seeing a problem." Then Lori reasoned about the concept and explained that, "as long as from that [index] and beyond it satisfies that condition, [...] I don't think it would matter where along the constant portion that you pick your $L "$ ". At the end of the session, Ada and Lori decided to keep their definition (see Figure 10) seemingly because they decided that there were two equivalent conceptualizations of the concept. One emphasized the first index value in which all the subsequent terms were equal and the other did not require identifying the minimal $L$.

The previous example shows that students might imagine a 'key one' when they use 'there exists'. While the students ended up deciding to keep their definition, Ada at least originally thought that they could (and should) alter the grammar of their definition so that it fit their thinking about it. 


\section{Grammar of 'where' and commas}

In the following section I will discuss how the structural aspects of 'where' was important to students' construals of AE and EA statements. Then, I discuss how describing an EA statement as first using an existential quantifier and then a universal quantifier is an insufficient description of EA statements.

The grammar of 'where' has a significant role in the construed meaning of

EA and AE statements. During the last session with Ada and Lori, I asked them to interpret the following:

A sequence $\left\{a_{k}\right\}$ is prime if for every prime number $p$ in $\mathbb{P}$, there exists at least one input $k$ where $a_{k}=p$.

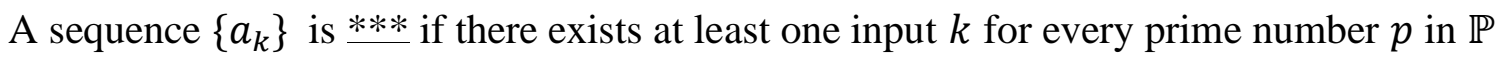
where $a_{k}=p$.

The two statements contain the same phrases but in different orders. Ada and Lori's definition for Prime sequence first stated 'for every prime number $p$ in $\mathbb{P}$ ' and then 'there exists at least one input $k$ '. I crafted the "Star" statement so that it first read 'there exists at least one input $k$ ' and then 'for every prime number $p$ in $\mathbb{P}$ '. The phrase 'where $a_{k}=p$ ' was last in both statements. This statement provides a communication test of the students' thinking regarding the grammar of 'where'.

Ada and Lori described a prime sequence as they had before. Lori explained that the statement conveyed "all of the prime numbers - two, three, five - all of them are in the sequence at least once." However, they were troubled by the Star definition. Lori explained, "It's coming from how my brain keeps reading it differently every time that I 
read it. I'm like 'that's fine' and then I'm like 'oh wait, no!'.” Ada agreed and Lori continued to explain:

“So, it's because I can sort of think of it there's a $k$ for every $p$. So, like here's $p_{1}$ oh it has its little $k$ buddy there, and here's $p_{2}$ and oh he's got a $k_{2}$. He's so happy - he's got a $k$. (See Figure 12A.) And that's nice because then you get like: $k_{1}, k_{2}, p_{1}, p_{2}$. Yay, they're happy! (See Figure 12B.) But then I can read it again and see there is one $k$ for every prime number where $a_{k}=p$. And then it's back to this big ugly guy so I think that means it's a bad definition (See Figure 12C)."

Notice that Lori first described iteratively pairing each prime number with an input $k$ ("here's $p_{1}$ oh it has its little $k$ buddy there, and here's $p_{2}$ and oh he's got a $k_{2}$. He's so happy - he's got a $k . ")$ Then she described an alternate possibility that one particular input $k$ such that $a_{k}=p$ for every prime number $p$. Moreover, she thinks that the Star statement is a "bad definition" because it can be interpreted either way.

I argue that it is the placement of 'where' that causes this confusion. The mathematics community suggests that where' (or 'such that') goes together with 'there exists' (Houston, 2009). I suggest that the reason for this is because 'where' (or 'such that') functions as an indicator for what must be true about the variables that were previously introduced. For instance, the prime definition conveys each prime number $p$ is iteratively paired with some natural number $k$ such that the following must be true about $p$ and $k: a_{k}=p$. However, even though the existential quantifier comes before the universal quantifier in the star statement, the signal to evaluate the statement (i.e. 'where') comes after the universal quantifier. As Lori described, this allowed the interpreter to consider the iterative pairings of $k$ and $p$. This example illustrates that the 
order of the quantifiers is not the only aspect of the grammar of a statement with multiple quantifiers that contributes to the construed meaning.

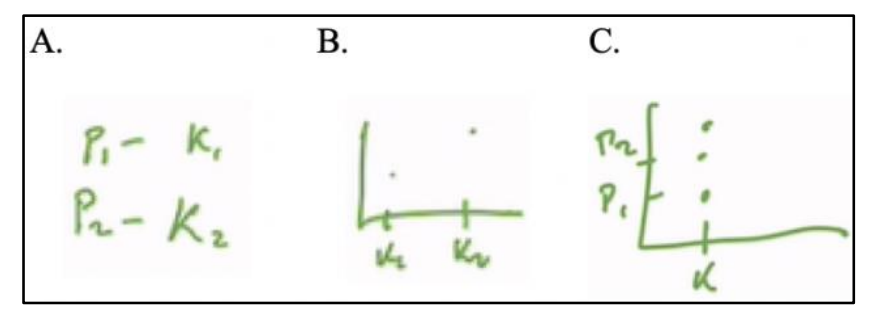

Figure 12. Lori's illustrations of Star interpretations

The research literature on AE and EA statements has focused on the order of quantifiers (and corresponding variables). By working with students, we see that the placement of 'where' (or equivalently 'such that') has a critical function. In particular, in an $\mathrm{AE}$ or EA statement it is an indicator that what is to follow is true about the previously introduced variable(s). In the case of 'For all $x \in X$, there exists $y \in Y$ such that $R(x, y)$ ' the 'such that' (and equivalent phrases) is the indicator that one is allowed to take multiple turns and play a different $y \in Y$ for each of the previously played $x \in X$.

\section{Concluding Remarks}

Here, I return to my two main points: (1) grammatical complexities influenced the students' construals of mathematical statements with quantifiers, and (2) these complexities highlight limitations for the ways that prior research has conceptualized statements with multiple quantifiers.

\section{Students' thinking about grammatical complexities}

My findings agree with prior studies that students' ability to construe the conventional interpretations of statements with multiple quantifiers depends on students' 
understanding of the syntax (Dawkins \& Roh, 2019; Dubinsky \& Yiparaki, 2000). Unlike prior studies, I found that there were other grammatical features that influenced the students' construals other than the order of the quantifiers. In particular, they considered 'for all' as different than 'for any' or 'for every' and this difference significantly affected the construals of AE statements. They also considered 'there exists' as different than 'there exists at least one'. The students considered the grammar of 'where' as important to convey the intended meaning of the statement.

More research is needed to (1) understand additional grammatical complexities that impact students' construed meanings, and (2) the extent to which the grammatical complexities help explain variations in student interpretations of statements with quantifiers. Dawkins and Roh's (2019) reports of students' responses on their survey suggest that the collective construal of 'for all' might be a reason for students' nonstandard interpretations of AE statements. They reported differences in student survey responses that could be predicted with the collective construal.

This study also exemplifies students' thoughtfulness in regards to their interpretation processes. This is in contrast to Dubinsky and Yiparaki's (2000) reports of their participants' inattention to their interpretation processes and failures to consider different interpretations of a given statement. The students in this study were able to deeply engage in thinking about their interpretation process and leverage their process to consider multiple interpretations of mathematical statements. The different result is likely to be because of the nature of the data. In this study, I engaged students in writing mathematical statements in such a way that supported students to focus on their language. 
This study also highlights that mathematical meanings can get lost in translation. Peirce, an American philosopher who is considered one of the founders of semiotics, speaks to Halliday's (2009) point that a shared contextual meaning is necessary for successful communication. Peirce wrote in a letter discussing his theory of signs:

"A man, tramping along a weary and solitary road, meets an individual of strange mien, who says, 'There was a fire in Megara.' If this should happen in the Middle United States, there might very likely be some village in the neighborhood called Megara. Or it may refer to one of the ancient cities of Megara, or to some romance. And the time is wholly indefinite. In short, nothing at all is conveyed, until the person addressed asks, 'Where?' - 'Oh about half a mile along there' pointing to whence he came. 'And when?' 'As I passed.' Now an item of information has been conveyed, because it has been stated relatively to a well-understood common experience." (published in Chandler, 2017, p. 478).

This story can be viewed as an analogy for a student and another person (e.g. a mathematician, teacher, or even another student). For example, consider a teacher and a student that did not have a "well-understood common experience" that the student held a collective meaning of 'for all' and the student used a 'for all $x \in X$, there exists $y \in Y$ such that, $R(x, y)^{\prime}$ to describe that there is one $y^{*} \in Y$ such that $R\left(x, y^{*}\right)$ is satisfied for every $x \in X$. Then the teacher might mistakenly think that a student does not understand the concept rather than identify the problem being how to conventionally describe the concept. For another example, consider a teacher and student that did not have a "wellunderstood common experience" that 'there exists' was synonymous with 'there exists at least one'. The student might draw conclusions about a mathematical statement that were not intended by the teacher. This was exemplified in Ada and Lori's interpretations of type-2 (onto) functions. To Ada, 'for all $y$ in $Y$ there exists a $x$ in $X$ such that $y=f(x)$ ' meant that each $y$ in $Y$ could only be mapped to by exactly one $x$ in $X$, which is not 
normative construal of the statement. It is critical that we support students in learning the (complex) language of mathematics by leveraging a well-understood common experience so that they can meaningfully participate in their class and communicate with the larger mathematics community.

\section{Advancing our understanding of $A E$ and $E A$ statements and the standard construals}

The research literature has traditionally used static ways of thinking about the language that is used in statements with multiple quantifiers and has focused on parts of the statements as the defining features of the intended meaning. However, understanding the complexities of such statements is more than simply understanding the sum of their parts. Here, I complement the static view of statements with multiple quantifiers with a systems view, adding to our understanding of the dynamical system as a whole. This view grew out of the need to make sense of the students' thinking about the grammar of the statement.

The students' considerations of 'for all' and the need for 'where' give us insight about the standard use of AE and EA statements. First, this study confirms that 'for all $x$ in $X^{\prime}$ in an $\mathrm{AE}$ statement is used to refer to each member of the set $X$ rather than the whole set $X$. This is because considering each member of the set $X$ seems to be what contributes to normative construals of AE statements. Additionally, the grammar of 'where' plays a crucial role in construing meaning from AE and EA statements. The function of 'where' (or equivalent) is an indicator for the interpreter that what is to follow is true about the previously introduced variables with their potential dependencies. 
Consider the following constructed generic statements with 'for all $x \in X$ ' and 'there exists $y \in Y^{\prime}$ :

AE Statement: For all $x \in X$, there exists $y \in Y$ such that $R(x, y)$.

EA Statement: There exists $y \in Y$ such that for all $x \in X, R(x, y)$.

Non-standard Statement: There exists $y \in Y$ for all $x \in X$ such that $R(x, y)$.

Using the dynamic construals, one interprets the following:

AE Statement: Iteratively take an $x \in X$ and find a $y \in Y$ such that the following is true about the $x-y$ pair: $R(x, y)$. This continues until every member of the set $X$ has been paired with a $y \in Y$.

EA Statement: There is a $y^{*} \in Y$ such that the following is true about $y^{*}: R\left(x, y^{*}\right)$ for every $x \in X$.

Non-standard Statement: Iteratively take an $x \in X$ and find a $y \in Y$ such that the following is true about the $x-y$ pair: $R(x, y)$. This continues until every member of the set $X$ has been paired with a $y \in Y$.

Notice that the AE statement and the non-standard statement construe the same meaning, even though the non-standard statement first introduces an existentially quantified variable and then a universally quantified variable. The subtle change of moving 'such that' after 'for all $x \in X$ ' changed the construed meaning from the normative construal of the EA statement to the normative construal of the AE statement. Attention to the function of 'such that' gives insight into why the order of the quantifiers matter. The ordering of the quantifiers in the EA Statement require the 'such that' to appear before introducing $x \in X$ and so $y^{*}$ must be fixed before evaluating $R\left(x, y^{*}\right)$ for 
every $x \in X$. Whereas the ordering of the quantifiers in the AE Statement require 'such that' to appear after both quantified variables are introduced, which allows more dynamic pairings $x$ 's and $y$ 's before evaluating $R(x, y)$.

To conclude, mathematical language, while intended to be clear and precise, is filled with nuances. Small differences in the language can lead differences in the construed meaning. I view this work as not only a step toward better understanding students' thinking about statements with quantifiers, but also progress for our own understanding, as researchers, of such statements. 


\section{References}

Alcock, L. (2012). How to study for a mathematics degree. OUP Oxford.

Bateson, G. (1979). Mind and nature: A necessary unity (Vol. 255). New York: Bantam Books.

Batty, C., \& Woodhouse, N. (1994). How do undergraduates do mathematics?. Mathematical Institute Study Guide.

Dawkins, P. C., \& Roh, K. H. (2019). Assessing the influence of syntax, semantics, and pragmatics in student interpretation of multiply quantified statements in mathematics. International Journal of Research in Undergraduate Mathematics Education, 1-22.

Dubinsky, E., \& Yiparaki, O. (2000). On student understanding of AE and EA quantification. Research in Collegiate Mathematics IV, 239-289.

Durand-Guerrier, V., \& Arsac, G. (2005). An epistemological and didactic study of a specific calculus reasoning rule. Educational Studies in Mathematics, 60(2), 149-172.

Halliday, M. (1978). Language as social semiotic: The social interpretation of language and meaning. London: Edward Arnold.

Halliday, M. (1996). Some grammatical problems in scientific English. In M. Halliday, \& J. R. Martin (Eds.), Writing science: Literacy and discursive power (pp. 76-94). London: Falmer Press.

Halliday, M. A. K. (2009). Language and Society: Volume 10. Bloomsbury Publishing.

Halliday, M. \& Matthiessen, C. M. (2013). Halliday's introduction to functional grammar. Routledge. 
Houston, K. (2009). How to think like a mathematician: A companion to undergraduate mathematics. Cambridge University Press.

Lemke, J. L. (2003). Mathematics in the middle: Measure, picture, gesture, sign, and word. Educational perspectives on mathematics as semiosis: From thinking to interpreting to knowing, 1, 215-234.

Lew, K., \& Mejía-Ramos, J. P. (2015). Unconventional uses of mathematical language in undergraduate proof writing. In Proceedings of the 18th Annual Conference on Research in Undergraduate Mathematics Education. Pittsburgh, PA.

Lew, K., \& Mejía-Ramos, J. P. (2019). Linguistic conventions of mathematical proof writing at the undergraduate level: Mathematicians' and students' perspectives. Journal for Research in Mathematics Education, 50(2), 121-155.

Gebhard, M., \& Martin, J. (2011). Grammar and literacy learning. Handbook of research on teaching the English language arts, 297-304.

Moore, R. C. (1994). Making the transition to formal proof. Educational Studies in Mathematics, 27(3), 249-266.

O'Halloran, K. (2008). Mathematical discourse: Language, symbolism and visual images. A\&C Black.

O’Halloran, K. L. (2015). The language of learning mathematics: A multimodal perspective. The Journal of Mathematical Behavior, 40, 63-74.

Roh, K. H. (2010). An empirical study of students' understanding of a logical structure in the definition of limit via the $\varepsilon$-strip activity. Educational Studies in Mathematics, 73(3), 263-279. 
Roh, K. H., \& Lee, Y. H. (2011). The Mayan activity: A way of teaching multiple quantifications in logical contexts. Problems, Resources, and Issues in Mathematics Undergraduate Studies, 21(8), 685-698.

Schleppegrell, M. J. (2007). The linguistic challenges of mathematics teaching and learning: A research review. Reading \& writing quarterly, 23(2), 139-159.

Strand, S. R. (2016). The Intermediate Value Theorem as a Starting Point for InquiryOriented Advanced Calculus.

Veel, R. (1999). Language, knowledge and authority in school mathematics. Pedagogy and the shaping of consciousness: Linguistic and social processes, 185-216.

Weber, K. (2001). Student difficulty in constructing proofs: The need for strategic knowledge. Educational studies in mathematics, 48(1), 101-119. 


\title{
Chapter 3: A Case Study of Gaining Fluency with Mathematical Language by way of Defining
}

\begin{abstract}
Statements with multiple quantifiers are characteristic of many advanced mathematics definitions and theorems; however, students struggle with interpreting and using such statements. This paper is a case study of a pair of students reinventing dynamical processes that are encoded in statements with multiple quantifiers. This paper is a proof of concept for an instructional approach that supports students to develop mathematical meanings, encode their meanings with statements with multiple quantifiers, and then leverage this experience to learn about statements with multiple quantifiers. I conclude with detailing initial steps toward articulating a local instructional theory that frames the students' reinvention process in terms of the emergent models construct from the theory of Realistic Mathematics Education.
\end{abstract}

Keywords: Defining, quantification, emergent models, case study

\section{Introduction}

Statements with multiple quantifiers are characteristic of many advanced mathematics definitions and theorems. However, it is well documented that students have difficulties with statements with multiple quantifiers despite their pervasiveness in advanced mathematics (e.g. Dawkins \& Roh, 2019; Dubinsky \& Yiparaki, 2000; DurandGuerrier \& Arsac, 2005; Roh, 2010; Roh \& Lee, 2011). In response, there have been several scholars who have designed instructional tasks or tools focused on promoting understanding of statements with multiple quantifiers (e.g. David, Roh, \& Sellers, 2018; Dubinsky \& Yiparaki, 2000; Dawkins \& Roh, 2016; Roh \& Lee, 2011). 
Here, I present a descriptive case study of students engaging in an alternate approach. The students in the present study engaged in defining (Zandieh \& Rasmussen, 2010) concepts that were meaningful to them and then reflected on the grammar for the resulting set of definitions. This approach for supporting students to learn about mathematical language, and particularly the grammar of statements with multiple quantifiers, is quite different than trying to make sense of statements that express some other individuals' meanings that have been formally encoded using quantifiers. Rather than working with unknown meanings and unfamiliar language rules, the students build an understanding of the grammar by trying to communicate their own meanings. Additionally, by connecting their mathematical meanings to more formal language, students should be better equipped to interpret statements with multiple quantifiers because they have the experience of (1) expressing ideas that are meaningful to them and (2) creating meaning from the grammar that they used to express their ideas.

\section{Literature Review}

In the following section, I first describe how research literature has conceptualized statements with multiple quantifiers. I then review the research literature on students' understandings of statements of multiple quantifiers and instruction designed to support students' learning about such statements. I compare the instructional approach that I present here to the approaches found in the research literature. 


\section{Conceptualizations of statements with multiple quantifiers}

There are two types of statements with multiple quantifiers that have received significant attention from the research community. Below are the generic forms of these statements with $R(x, y)$ representing some relation between $x$ and $y$.

AE statements: For all $x \in X$, there exists $y \in Y$ such that $R(x, y)$.

EA statements: There exists $y \in Y$ such that for all $x \in X, R(x, y)$.

In Chapter 2, I reinterpreted Dubinsky and Yiparki’s (2000) game that was designed to support students in interpreting AE and EA statements as dynamic descriptions of the conventional construals. These construals are systems that describe how and when to select $x \in X$, select $y \in Y$, and evaluate $R(x, y)$. In particular, when an interpreter reads 'for all $x \in X$, there exists $y \in Y$ such that $R(x, y)$ ' they consider iteratively taking an $x \in X$ and finding a $y \in Y$ such that $R(x, y)$ is true for the selected pair and they continue this process until every member of the set $X$ has been paired with a $y \in Y$. That is, take $x_{1} \in X$ and then find an $y_{1} \in Y$ such that $R\left(x_{1}, y_{1}\right)$, then select $x_{2} \in$ $X$ and then find an $y_{2} \in Y$ such that $R\left(x_{2}, y_{2}\right)$, and continue this process for every member of the set $X$. I call this process for selecting $x \in X$, selecting $y \in Y$, and evaluating $R(x, y)$ in in this way as the AE Process. Another way to view the meaning of an AE statement is with a static pairwise relationship. Dawkins and Roh (2019) explain that $\mathrm{AE}$ statements describe each-to-some relationships; each $x \in X$ is associated with some $y \in Y$ in such a way that $R(x, y)$ holds. I view this way of conceptualizing an $\mathrm{AE}$ statement as a snapshot of the dynamic process of the AE Process. 
Alternatively, when an interpreter reads 'there exists $y \in Y$ such that for all $x \in$ $X, R(x, y)^{\prime}$ they consider finding a $y^{*} \in Y$ such that $R\left(x, y^{*}\right)$ for every $x \in X$. I call this type of process an EA Process. Dawkins and Roh (2019) suggest that EA statements describe a one-to-every relationship; one $y \in Y$ corresponds with every $x \in X$ in such a way that $R(x, y)$ holds. Again, I view this static pairing as a snapshot of the dynamic process of the EA Process.

\section{Students' Thinking about Statements with Multiple Quantifiers}

It is well documented in the research literature that students have difficulties with statements with multiple quantifiers (Dawkins \& Roh, 2019; Dubinsky \& Yiparaki, 2000; Durand-Guerrier \& Arsac, 2005; Roh, 2010; Roh \& Lee, 2011). Some of the reported difficulties include recognizing relationships between the universally quantified variable and the existentially quantified variable. For instance, in AE statements students may not regard the existentially quantified variable as potentially dependent on the universally quantified variable (Durand-Guerrier \& Arsac, 2005; Epp, 2009) or may not regard the universally quantified variable as independent of the existentially quantified variable (Roh, 2010; Roh \& Lee, 2011). This not only limits students' ability to construe the conventional interpretation of statements with multiple quantifiers but also limits students' proving activity involving these statements (Durand-Guerrier \& Arsac, 2005; Roh \& Lee, 2011). For instance, Roh and Lee (2011) presented two cases where the students did not treat $\varepsilon$ as independent of $N$ when applying the $\varepsilon-N$ definition of sequence convergence in proofs (A sequence $\left\{a_{n}\right\}$ is converges to a real number $L$ if for any positive real number $\varepsilon$, there exists a natural number $N$ such that for all $n>N$, 
$\left.\left|a_{n}-L\right|<\varepsilon\right)$. This caused the students to fail to construct a valid proof or arrive at a proper conclusion to the given problem. The authors pointed to the students' understanding of the syntax of the statement, and specifically the role of the order of the quantifiers, as reason for their proving mistakes.

There are two studies that have focused on investigating students' thinking as they interpreted statements with multiple quantifiers (Dawkins \& Roh, 2019; Dubinsky \& Yiparaki, 2000). Dubinsky and Yiparaki (2000) surveyed and interviewed students about their interpretations of several statements that were in the natural language context (e.g. 'Every pot has a cover') and two statements that were in the same mathematical context (e.g., For every positive number $a$ there exists a positive number $b$ such that $b<a$.) Each of the statements in the natural language were also considered AE or EA depending on the intended meaning. For instance, 'every pot has a cover' was coded as an AE statement because the intended meaning was that each pot is associated with some cover. Dubinsky and Yiparaki (2000) found that the students did not tend to use the syntax of the statements to make sense of the statements but rather relied on their view of the world. The students were likely to interpret EA statements as AE statements. Additionally, the students were less likely to construe the intended meaning of the mathematical statements than the statements in the natural context.

Dawkins and Roh (2019) conducted a study to further investigate students' interpretations of mathematical statements with multiple quantifiers. They surveyed 77 students in Introduction to Proof courses to explore how (a) syntax, (b) the reader's understanding of the ideas referenced in a statement (i.e. semantics), and (c) the 
plausibility or relevance of an interpretation (i.e. pragmatics) influenced students' interpretations. The survey instrument included eight statements (four of which were true) in the context of two content domains (i.e. function and geometry). They found that semantics played an important role in student interpretations and, more specifically, that the patterns of their students' interpretation relied more on the mathematical context than on the order of the quantifiers. This reliance sometimes led students to interpret the statements in a conventional way, but other times led them to unconventional interpretations. They also found that students' attention to pragmatics influenced their interpretations. In particular, students had more difficulty with statements that were less interesting (e.g. for all real numbers $x$, there exists a real number $M$ such that $3 x+2<$ $M$ ) or relatively absurd (e.g. There exists a point $C$ on the segment $\overline{A B}$ such that for every positive real number $s, d(A, C)=s)$. Additionally, they found that the students were likely to become more sensitive to the order of the quantifiers after they completed the course (though they did not offer any information about the instruction that the participants experienced).

There are several key takeaways from my review of the literature on student thinking about statements with multiple quantifiers. First, the syntax alone is not likely to give novices insight about the intended meaning of a mathematical statement with multiple quantifiers. Rather, students tend to leverage semantics and pragmatics to interpret statements with multiple quantifiers, which can lead them astray especially if they are also not attending to syntax. However, students are likely to become more sensitive to the syntax of a statement over the course of an Introduction to Proofs course. 
The question of what characterizes instruction that supports students in becoming more attuned to the syntax remains unanswered.

\section{The Teaching of Statements with Multiple Quantifiers}

I identified two research papers about two instructional approaches during my review of the research literature on the teaching of statements with multiple quantifiers (Dawkins \& Roh, 2016; Dubinsky \& Yiparaki, 2000). The first approach aimed to leverage the syntax of the statement (Dubinsky \& Yiparaki, 2000) while the second intended to promote an understanding of the statements with multiple quantifiers by offering carefully designed analogies (Dawkins \& Roh, 2016). In what follows, I will describe these approaches in more detail and then contrast them with the approach that I describe in the present study.

Dubinsky and Yiparaki’s (2000) approach was motivated by their findings related to students' interpretations of statements with multiple quantifiers. In particular, they reasoned that using natural language as a starting point for supporting students to learn about mathematical statements with multiple quantifiers was not a productive approach. They explained that the natural language does not follow the same strict guidelines as mathematical language and so one cannot take advantage of something that may not be there. For instance, one cannot use the different structures of 'there is a mother for all children' and 'all children have the same mother' to highlight different meanings since students are likely to think that both statements convey the same meaning - every child has a mother. They advise focusing on teaching the syntax of mathematical statements without reference to the natural language as a way to support students in gaining an 
understanding about the intended meaning, and offer an intervention in the form of a twoplayer game.

Dubinsky and Yiparaki’s (2000) game assigns players to either a universal quantifier role (Player A) or else an existential quantifier role (Player E) and then the syntax of a given statement dictates the players' permittable moves and the game winner. To exemplify, consider the following statements:

(True) AE statement: For any real number $x$, there exists a positive integer $m$ such that $m>x$.

(False) EA statement: There exists a positive integer $m$ such that for any real number $x$, $m>x$.

To play the game, Player A selects real numbers and attempts to establish that the relation is false (e.g., $m \leq x$ ). Player E selects positive integers and attempts to establish that the relation is true (e.g., $m>x$ ). In the case the players are given the AE statement, Player A starts the game by selecting a real number. Since Player A is trying to establish the relation is false, their strategy is to find a real number such that no matter what positive integer Player E selects, the positive integer will not be greater than the real number. Once Player A selects a real number, then Player E attempts to find positive integer such that $m>x$. And the game continues in this way. Of course, it is impossible for Player A to find a real number such that the real number is greater than or equal to all the possible positive integers and so Player E will win the game. In fact, in the case that the given statement is an AE statement, Player A can only be successful if it is false. In the case that the players are given the EA statement, Player E takes the first turn and 
attempts to select a positive integer such that no matter what real number Player A chooses, the positive integer is greater than the real number (that is, the relation described in the statement is true). During Player A's turn, they attempt to find a real number such that relation is false. If Player A is successful (as they should be with the above EA statement), then they win. In this way, Player E should be the winner when given a true EA statement.

Dubinsky and Yiparaki (2000) reported cases in which the game supported the students in gaining an understanding of the syntax of statements. For instance, a student who had previously interpreted $\mathrm{AE}$ and $\mathrm{EA}$ versions of a statement the same way, was able to see a distinction in the conveyed meanings after playing the game. However, they also reported times when the game did not seem to help or even caused further confusion for the students.

My approach is quite different than Dubinsky and Yiparaki's (2000) game intervention. Their study focuses on supporting students in interpreting mathematical statements, that is, the intervention was designed to support students in making sense of a given mathematical statement. In contrast, my approach supports students to (a) develop their mathematical meanings, (b) encode their meanings with more formal language, and then (c) leveraging this experience to learn about the grammar of statements with multiple quantifiers.

Dawkins and Roh's (2016) intervention aimed to promote students' understanding of mathematical conventions and assumptions, including understanding statements with multiple quantifiers, by utilizing well-crafted analogies. Their approach pushed back on 
Dubinsky and Yiparaki's (2000) advice to steer away from comparing mathematical language to natural language. They suggest that analogies from natural language can be a helpful tool when they are well thought out and rely on similar rules and conventions found in mathematics. Their study presented a case of students analyzing "Bill's argument" which was an invalid proof that concluded $\left\{\frac{1}{n}\right\}$ did not converge to 0 (see Figure 13). Dawkins and Roh (2016) reported that during the students' initial reading they identified that Bill's argument concluded a false statement but struggled to identify the flaw in the argument (that $\varepsilon$ depended on $N$ ). The authors described Bill's argument as assuming a common interpretation of the sequence convergence definition incompatible with mathematical convention.

Bill's argument. For all $N \in \mathbf{N}$, choose $\varepsilon=\frac{1}{(N+2)}$. Then $\varepsilon>0$ since $N>0$. Let $n=N+1$. Then $n>N$. Also, $\left|\frac{1}{n}-0\right|=\frac{1}{n}=\frac{1}{(N+1)}>\frac{1}{(N+2)}=\varepsilon$. Therefore, $\left\{\frac{1}{n}\right\}$ does not converge to 0 .

Figure 13. Invalid proof that $\left\{\frac{1}{n}\right\}$ did not converge to 0 (from Dawkins \& Roh, 2016, p. 208).

Then, the teacher in Dawkins and Roh's (2016) study introduced the Mayan stonecutter story to support students to reason about the relationships among the variables $\varepsilon, N$, and $n$ in the $\varepsilon-N$ definition of sequence convergence and its negation. See Figure 14. The definition states: A sequence $\left\{a_{n}\right\}$ converges to a real number $L$ if for any positive real number $\varepsilon$, there exists a natural number $N$ such that for all $n>N$, $\left|a_{n}-L\right|<\varepsilon$. In the story, the priest's requested margin of error is analogous to $\varepsilon$ in the definition, the craftsman's requested number of days is analogous to $N$, and the gap between stones at the $n_{\text {th }}$ day is analogous to $\left|a_{n}-L\right|$. 


\begin{abstract}
The Mayan stonecutter story. One of the famous Mayan architectural techniques is to build a structure with stones. These stones were ground so smoothly that there was almost no gap between two stones. It was even hard to put a razor blade between them. One day a priest came to a craftsman to request smooth stones.

Craftsman: No matter how small a gap you request, I can make stones as flat as you request, if you give me some time.

Priest: I do not believe you can do it. If I ask you to flatten stones within $0.01 \mathrm{~mm}$, you won't be able to do it.

Craftsman: Give me 10 days, and you will receive stones as flat as within $0.01 \mathrm{~mm}$.

Ten days later, the craftsman made two stones so flat that the gap between them was within $0.01 \mathrm{~mm}$. On the 11th day, the priest came to see the stones and argued,

Priest: These stones are not flat within $0.001 \mathrm{~mm}$. What I actually need are stones as flat as within $0.001 \mathrm{~mm}$.

Craftsman: Okay, if you give me 5 more days, I can make the stones as flat as within $0.001 \mathrm{~mm}$.

Five days later, the craftsman made the two stones so flat that the gap between them was within $0.001 \mathrm{~mm}$. On the 16th day, the priest came to see the stones and argued,

Priest: But these stones are not flat within $0.0001 \mathrm{~mm}$ and I meant $0.0001 \mathrm{~mm}$. You don't have that kind of skill, do you?

If the priest keeps arguing this way, is the priest really fair showing that the craftsman does not have the ability to flatten stones within any margin of error?
\end{abstract}

Figure 14. The Mayan stonecutter story (from Roh \& Lee, 2011, p. 694).

Dawkins and Roh (2016) explain that key to the Mayan stonecutter story is that the priest's argument was unfair. The craftsman promised that he could make the gap as small as the priest would like if the priest gave him enough time. The priest's argument claimed that the craftsman was unable to fulfil his promise because the craftsman had not smoothed the stones to a newly requested margin of error (ignoring that a requested smoothness depended on the required amount of time). Dawkins and Roh (2016) claimed that the students were able to identify the relevant structural differences in the craftsman's argument and the priest's counterargument: the priest's argument did not negate the craftsman's claim because the priest reversed the order of the quantities 
imposing a single time for all margins of error rather than a distinct work time for each margin of error.

Dawkins and Roh (2016) reported that the Mayan stonecutter story shifted the students' attention to the quantification order and the dependency involved with Bill's argument. They argued that the story gave students the language to describe the relationships among the variables in the definition. The students were able to articulate how the order of introducing variables led to (in)dependent relationships by leveraging how the stonecutter and priest determined their requirements for smoothness.

Additionally, Dawkins and Roh (2016) reported that the Mayan stonecutter story became a paradigm for the students when they later leveraged the story to make sense of arguments involving statements with multiple quantifiers.

Underlying Dawkins and Roh's (2016) analogy intervention is the assumption that the analogies have a shared meaning, that is, the students understand the intended meaning of the story, allowing them to focus on the language itself. My approach is similar in this aspect in that the students in my study were first supported to develop a shared understanding of the concept before attempting to write a definition. A difference in Dawkins and Roh's intervention and the one that I propose here is that my intervention is in the mathematical context and not an analogy. I view the approach that I presented in this study as a more authentic approach to learning about mathematical language.

In the present study, I investigate the feasibility of starting with supporting students to develop students' mathematical meanings, encode their meanings with more formal language, and then leveraging this experience to making sense of the grammar of 
statements with multiple quantifiers. Drawing on the theory of Realistic Mathematics Education, I conducted a series of design experiments in order to investigate how students might be supported in learning how to use mathematical language to define concepts that can be expressed in terms of EA or AE Processes. In this paper, I present a case study of two students engaged in this kind of mathematical activity to 1) provide a proof of concept for this approach and 2) begin the process of articulating a local instructional theory that frames students' reinvention of EA and AE Processes as emergent models.

\section{Theoretical Grounding}

In the following section, I will first discuss the instructional design theory that guided my task design and retrospective analysis. Then, I will further discuss my conceptualization of the instructional approach as engaging students in defining and in a model eliciting activity.

\section{Realistic Mathematics Education}

The theory that underlies this study is Realistic Mathematics Education (RME). $\mathrm{RME}$ is an instructional design theory that is based on the principle that "mathematics is a human activity" meaning that, "mathematics can and should be learned on one's own authority and through one's own mental activities" (Gravemeijer, 1998, p. 277). Guided reinvention is the overarching design heuristic of any RME design study requiring students to have the opportunity to construct their own knowledge in a way that they come to see it "as their own, personal knowledge, knowledge for which themselves are responsible" (Gravemeijer \& Terwel, 2000, p. 786). Guided reinvention played at least 
two distinct roles in my efforts to design instruction to support students in learning to write and interpret statements with multiple quantifiers. First, the students were engaged in reinventing definitions for two particular concepts which we called 'Eventually Constant Sequence' and 'Prime Sequence'. Second, students were engaged in reinventing EA and AE Processes. The general principle of my instructional approach is that reinventing the two concepts would support students in reinventing the $\mathrm{EA}$ and $\mathrm{AE}$ Processes. The focus of this paper is on students' EA and AE Processes which I argue was made possible by reinventing definitions for Eventually Constant Sequence and Prime Sequence.

While guided reinvention is the overarching goal of any RME design study, other heuristics like emergent models can give more specific guidance for a design researcher in design and analysis (Larsen, 2013). The 'model' in emergent models refers to a mathematical concept that the students reinvent. For my investigation, I considered two models: the EA and AE Processes. Using emergent models, formal mathematics is viewed as growing out of students' activity. Specifically, students "develop formal mathematics by way of mathematizing their own informal mathematical activities" (Gravemeijer, 1999, p.160) and by 'mathematizing' Gravemeijer means organizing from a mathematical perspective (Gravemeijer \& Doorman, 1999). In my study, the students develop an understanding of the grammar of statements with multiple quantifiers by way of mathematizing their own informal ideas about EA and AE Processes. The two models coemerged as the students' mathematizing their informal ideas about the EA and AE Processes. In the following paragraphs, I will describe how emergent models can guide 
task design and analysis in an RME study and how specifically it supported me in these ways.

As a design heuristic, emergent models advises the designer to imagine a path in which formal mathematics might emerge from informal understandings. In particular, the designer imagines how they might evoke students' informal understandings related to the desired concept and then how students might leverage their ideas to support the development of the formal concept. I hypothesized that engaging students in defining concepts that could be described with EA and AE Processes would evoke students' informal thinking about these processes. In this study, the students engaged in defining Eventually Constant Sequence and Prime Sequence.

The students in my study understood Eventually Constant Sequence to be a sequence that had an index value such that the index value and all the index values after it mapped to the same real number. Using an EA Process, there is a $L^{*} \in \mathbb{N}$ such that $a_{k}=$ $a_{k+1}$ for every $k \geq L^{*}$. This process can be encoded in an EA statement: there exists a natural number $L$ such that for all natural numbers $k \geq L, a_{k}=a_{k+1}$. Of course, this is not the only process (or statement) that one might construct when defining the concept. One could consider a process that describes an almost everywhere constant sequence (in the technical sense). That is, there is a $r^{*} \in \mathbb{R}$ such that $a_{k}=r^{*}$ for all but finitely many $k \in \mathbb{N}$. The students in my study conceptualized a Prime Sequence to be a sequence that contained all the primes. Using an AE Process, one selects a prime number $p_{1}$ and then finds an index value $k_{1}$ such that $a_{k_{1}}=p_{1}$, then selects a prime number $p_{2}$ and then finds an index value $k_{2}$ such that $a_{k_{2}}=p_{2}$, and so on until pairs have been found for every 
prime number. This process can be encoded with the following AE statement: for all prime numbers $p$, there exists a natural number $k$ such that $a_{k}=p$.

I conjectured that having students reflect on their definitions would support them in making the EA and AE Processes more explicit and so I designed a task in which the students would use their definitions to write an instruction manual for using universal and existential quantifiers. I will further discuss the theory of this design, and particularly what I mean by defining and the guiding principles of the instruction manual task, in the subsequent sections.

Emergent models can also be used as an analytic tool by framing how students' informal mathematical activity might develop into more formal mathematics. In particular, the researcher searches for instances in which the mathematical concept can explain the students' informal activity, but the students may not yet be aware that such a concept exists. Then the students become more aware of the concept as it becomes a tool for more general mathematical activity. There is a key shift when the students recognize and begin to make use of the mathematical idea. When this happens, we say that a modelof informal mathematical activity becomes a model-for more formal mathematical reasoning (Gravemeijer, 1999). Here, I am concerned with two model-of/model-for transitions: the model-of/model-for transition for EA Processes and the model-of/modelfor transition for AE Processes.

Another compatible way to investigate emergent models is with Gravemeijer's (1999) levels of activity. The goal in describing the levels of activity is to "illustrate that models are initially tied to activity in specific settings and involve situation-specific 
imagery" (Gravemeijer, 1999, p. 163). The four levels of activity include the situated, referential, general, and formal. Situational activity involves organizing a given problem in context (Gravemeijer, 1998). In this study, the task setting of the EA and AE models is the defining Eventually Constant Sequence and Prime Sequence, respectively. Referential activity includes reflecting on previous activity and general activity includes reasoning about the concept independently of situation-specific imagery (Gravemeijer, 1999). In this way, the model-of/model-for transition occurs when activity goes from referential to general. Additionally, formal activity includes reasoning in ways that reflect the emergence of a new mathematical reality and no longer requires the support of the model. The types of activity correspond to a developmental progression; however, the activity is not purely hierarchical. Gravemeijer explains that "discussions on the level of general activity and of formal mathematical reasoning frequently fold back to referential activity or even to activity in the setting" (1999, p. 163).

A third complementary way to use emergent models to investigate the emergence of a mathematical concept in the form of students' informal activity is through chains of signification (Cobb et al., 1997; Gravemeijer, 1999; Larsen, 2004). A chain of signification focuses on the students' refinements of symbolic representations (what are called signifiers) along with the meaning that the student associates with it (what is called signified). In a chain of signification, the representation is said to "slide under" the succeeding signifier (Gravemeijer, 1999), meaning that the new signifier encompasses the previous and takes on the previous sign's meaning. Gravemeijer explains that "a chain of signification is in a sense the counterpart, on a more specific level, of what the 
model is on a more general level" (1999, p. 174). Here, I leverage chains of signification by discussing the signifier-signified pairs related to EA Processes and signifier-signified pairs related to AE Processes.

\section{Defining as a Mathematical Activity}

My task design aimed to engage students in defining and then support them to capitalize on their experience to learn about EA and AE Processes. I adopt Zandieh and Rasmussen's (2010) conceptualization of defining which includes more than just creating a definition; defining can occur "as students are proving a statement, generating conjectures, creating examples, and trying out or 'proving' a definition" (p. 59). They leveraged the notions of concept image and concept definition to describe the activity of defining. A concept image is "the total cognitive structure that is associated with the concept, which includes all the mental pictures and associated properties and processes" and a concept definition is "words used to specify that concept" (Tall \& Vinner, 1989, p. 152). Zandieh and Rasmussen propose that creating a concept image, using a concept image, creating a concept definition, and using a concept definition are activities which constitutes defining.

In order to define Eventually Constant Sequence and Prime Sequence the students in my study created a concept image of the two concepts which involved generating and negotiating examples and non-examples of the concepts. Then, the students used their concept image to write down a concept definition. This concept definition was then refined using their concept image to evaluate their definition until the students were satisfied that their concept definition specified the concept. 


\section{Model-Eliciting Activity}

After the students defined Eventually Constant Sequence and Prime Sequence the students were asked to use their definitions to write an instruction manual for peers to use a universal quantifier and an existential quantifier when defining, and were specifically asked to fill in the banks:

$$
\begin{aligned}
& \text { Use 'for every } a \ldots \text { there exists } b \ldots \text { ' when: } \\
& \text { Use 'there exists } b \ldots \text { for every } a \ldots \text { ' when: }
\end{aligned}
$$

This task can be viewed as a model-eliciting activity (Lesh, Hoover, Hole, Kelly, \& Post, 2000). Lesh and his colleagues describe model-eliciting activities as tasks designed to encourage students to make sense of mathematical situations by inventing, extending, and refining their mathematical ideas (Lesh et al., 2000; Carlson, Larsen, Lesh, 2003). The product of a model-eliciting activity is a student-generated description that reveals the students' thinking. There are six guiding principles of model-eliciting activities. See Figure 15 for a list of the principles (as questions posed by, or adapted from, Lesh et al., 2000 or Carlson, Larsen, \& Lesh, 2003).

\begin{tabular}{|ll}
\hline Reality Principle & $\begin{array}{l}\text { Will students make sense of the situation by extending } \\
\text { their own knowledge and experiences? }\end{array}$ \\
$\begin{array}{l}\text { Model Construction } \\
\text { Principle }\end{array}$ & $\begin{array}{l}\text { Does the task immerse students in a situation in which } \\
\text { they are likely to confront the need to develop (or } \\
\text { refine, modify, or extend) a mathematically significant } \\
\text { construct? }\end{array}$ \\
Simplicity Principle & $\begin{array}{l}\text { Does the solution provide a useful prototype, or } \\
\text { metaphor, for interpreting other situations? }\end{array}$ \\
Self-Evaluation Principle & $\begin{array}{l}\text { Does the activity promote self-evaluation on the part of } \\
\text { the students? }\end{array}$
\end{tabular}




$\begin{aligned} & \text { Construction } \\ & \text { Generalization Principle }\end{aligned}$ this type of situation?
$\begin{aligned} & \text { Construct } \\ & \text { Wocumentation Principle the question require students to reveal their } \\ & \text { thinking about the situation? }\end{aligned}$

Figure 15. Six principles of model-eliciting activities.

I view model-eliciting activities as being directly tied to RME and that any task that was designed with the guided reinvention and emergent models heuristics in mind can be modified to be a model-eliciting activity. In particular, I propose that the Reality Principle is guaranteed to be satisfied by the guided reinvention heuristic and the Model Construction, Self-Evaluation, and Construct Generalization Principles are guaranteed by the emergent models design heuristic. Additionally, modifying the task so that it satisfies the Construction Documentation and the Simplicity Principles is one way to promote the model-of/model-for transition because these principles require the students to make the model explicit and present it in a simple enough way so that they can use the model for more general mathematical activity. Taking the six principles (and corresponding RME design heuristic) together, model-eliciting activities promote students to extend their experience in order to develop and then document their understanding of a mathematically significant construct. In this way, the students' informal activity evolves to be more general and powerful.

\section{Methods}

\section{Context and Conducting Design Experiment}

I conducted a laboratory design study with two community college students, Ada and Lori. By laboratory design experiment I mean a design experiment (Cobb \& 
Gravemeijer, 2008) with a pair of students, rather than in a regular classroom setting. Both students were recruited from an Introduction to Proofs course for which I was the teacher-researcher. This class was part of my research team's initial effort to scale up an inquiry-oriented curriculum in the context of introductory real analysis. During the course, the students reinvented several root-approximation methods and then followed Cauchy's lead by repurposing their methods to reinvent a proof-outline of the Intermediate Value Theorem (IVT). During the instructional sequence the students learned about several concepts in real analysis, including: The Intermediate Value Theorem, definition of monotone sequence, the Monotone Convergence Theorem, the definitions of supremum and infimum, and the Completeness Axiom.

There were a couple of reasons why I recruited Ada and Lori to participate in the laboratory design experiment. First, Ada and Lori were typically eager to share their thinking and they seemed to work well together during the Introduction to Proofs class. These were characteristics that I expected would help me to collect rich data on their thinking during the laboratory experiment. Secondly, at the end of the course neither student consistently used or interpreted mathematical statements with a universal quantifier and an existential quantifier correctly, which was typical of the students in the class. For instance, during the second to last day of the class, the class attempted to define a sequence that was unbounded above. One of Ada and Lori's classmates proposed the following statement, which I directed the students to consider as a possible definition: A sequence $\left\{a_{n}\right\}$ is unbounded above if and only if there exists a natural number $n$ where $a_{n}>K$ for any real number $K$. Conventionally, this statement would be interpreted as 
there is a natural number $n^{*}$ such that $a_{n^{*}}>K$ for every real number $K$. That is, there is an index that corresponds to a step in the sequence that is greater than every real number, which does not specify the concept of an unbounded above sequence. However, Lori was satisfied with this definition and explained that she thought it meant, "any number in the sequence is bigger than any number you can throw at it". I interpret Lori's explanation to mean that to her the statement meant that if you take any sequence term then the term is greater than any real number; that is, every sequence term is greater than every real number. This does not fit the conventional interpretation of the statement nor the expected concept image of unbounded above.

Additionally, there was a similar item for students to consider on an exit survey. Specifically, the students were asked the meaning of the following two statements: Statement A: For every real number $r$ there exists a natural number $n$ such that $n>r$. Statement B: There exists a natural number $n$ such that for all real numbers $r, n>r$.

Ada explained that the two statements "are the same sentence just flipped" and that both statements meant that "there will always be an $r$ less than a number $n$ ". I have two possible interpretations of Ada's response: (a) each real number $r$ will correspond to some natural number $n$ such that $r<n$, or (b) each natural number $n$ will correspond to some real number $r$ such that $r<n$. The first interpretation is consistent with the standard interpretation of Statement A, but inconsistent with the standard interpretation of Statement B. The second interpretation is inconsistent with the intended meaning of both statements. In either case, Ada's response suggests that she did not see the distinction between the two statements' meanings. 
Ada and Lori typically excelled in their undergraduate mathematics studies. Ada was in her first year at the community college and was considering double majoring in mathematics and music once she transferred to a university. In addition to the Introduction to Proof course, she completed the single-variable calculus courses offered at the community college. Lori was in her second year at the community college and planned to transfer to a university the following year to major in Physics. At the time of the study, she had completed all introductory mathematics courses offered by the community college (i.e. the full calculus sequence, Differential Equations, Linear Algebra). Neither student had formal proof experience at the undergraduate level prior to the course.

The laboratory design experiment spanned 11 sessions with each lasting approximately 1.5 hours. There were a couple of goals for the laboratory design experiment. One goal was to learn how to support students in using definitions to structure proofs. The other, which is the focus of this report, involved learning how to support students to gain fluency with mathematical language (and specifically with statements with multiple quantifiers by way of defining). The task sequence that I describe in this paper focuses on three sessions (Session 3, Session 9, and Session 11). The other sessions involved tasks related to the first goal. I served as the teacherresearcher and there was at least one witness during all of the sessions. The sessions were recorded on multiple devices; a video-camera captured Ada and Lori’s gestures and screen mirroring software video-recorded their written work on two tablets. 
Between sessions I conducted ongoing analysis. This analysis involved rewatching the previous session's video and writing a detailed chronological description of the students' activities and the conversations that took place. These descriptions were used to aid debriefing sessions between each teaching session with another researcher who was not present during the teaching session. This researcher read the descriptions and asked clarifying questions and then I amended the descriptions as needed. Then, we discussed and refined the task design for the following teaching session.

\section{Retrospective Analysis}

After the completion of the laboratory design experiment, I conducted retrospective analysis of the students' thinking and mathematical activity related to EA and AE Processes. I first reread the chronological descriptions that I created during my ongoing analysis and re-watched the corresponding video looking for ways of thinking that anticipated EA and AE Processes. Then, I aimed to identify the features of the task design or the discourse that evoked these ways of thinking. This approach is consistent with the analysis of other design researchers using RME to design tasks (e.g. Gravemeijer, 1999; Larsen, 2013; Larsen \& Lockwood, 2013). During this stage of the analysis, the emergent models design heuristic proved to be a powerful tool for describing students' productive and powerful ideas. Specifically, it seemed as though the defining tasks evoked ideas about EA and AE Processes and that Ada and Lori seemed to gradually take ownership of the processes creating various instantiations of the processes as they engaged in the tasks. To further investigate the emergence of the EA and AE Processes, I described the two chains of signification that emerged from Ada and Lori's 
defining activity, attempted to make sense of Ada and Lori's activity with Gravemeijer's (1999) levels of activity, and searched for evidence for model-of/model-for transitions. In what follows, I will describe my analysis in more detail.

First, I attended to describing the two chains of signification that emerged from Ada and Lori's defining activity. To do so, I took a picture of the students' work each time they made an inscription of what I could identify as an EA or AE Process. See the Appendix for the two chains of signification that documented the evolution of Ada and Lori's inscriptions of EA and AE Processes. Many of these inscriptions were in the form of definition refinements for Eventually Constant Sequence and Prime Sequence, but they included graphs and diagrams. In addition to documenting the inscriptions, I also recorded the timestamps and my interpretation of Ada and Lori's activity that led to each inscription. My interpretations were informed by both my data set as well as a mathematical analysis of the proposed definitions. During my mathematical analysis, I attempted to interpret the meaning of each definition given my knowledge of the standard conventions, paying attention to examples of the concept that the proposed definition excluded and non-examples of the concept that the definition included. This allowed me to gain an understanding of (mis)alignment between the proposed definition and the students' concept image and consequently what might have motivated the students' refinements. The chains of signification also allowed me to gain some insight about the evolution of the models. For example, I could see when their inscriptions evolved from representing a process in the initial task setting to when they used the process in another context. 
To further investigate the evolution of the models, I then analyzed Ada and Lori's activity in light of Gravemeijer's (1999) levels of activity searching for model-of/modelfor transitions. My goal was to identify when Ada and Lori were engaged in situational, referential, general, or formal activity. To identify Ada and Lori's situational activity, I searched for when they were organizing a given problem of defining Eventually Constant Sequence or Prime Sequence. Indicators of this activity were creating and using concept images (e.g., generating and leveraging examples and non-examples of the concepts) to create a concept definition of the two concepts. To identify referential activity, I searched for when they reflected on their previous defining activity. Indicators of this activity were instances when the students explicitly recalled a previous definition (or the defining activity) or when they explicitly reflected on their definition and the process that it represented. To identify general activity, I searched for when the students reasoned about the EA and AE Processes independent from their defining activity. Since the modelof/model-for transition occurs when the students are able to engage in general activity, I questioned if the students were aware of the EA and AE Processes as concepts that existed outside of Eventually Constant Sequences and Prime Sequences. Indicators of general activity where times when the students explicitly described the processes without a context, used the EA and AE Processes as a tool outside of defining Eventually Constant Sequences and Prime Sequences, respectively, or produced generalize inscriptions of the EA and AE Processes. I also searched for evidence of formal activity by searching for the students reasoning in ways that reflected a new mathematics reality that no longer required the support of the models (e.g., using their definitions in proof 
without explicit discussion about the underlying dynamical process encoded in the definition); but, as expected, I did not find any evidence of students engaging in it. In the following section I present the results of my analysis of the co-emerging models.

\section{Results}

In the following section, I present my analysis of the co-emerging models. In the first section, I discuss how the EA Process was evoked by engaging Ada and Lori defining Eventually Constant Sequence. In the second section, I discuss how the AE Process was evoked by engaging them in defining Prime Sequence and how the EA Process model was further developed. In the third section, I discuss how the EA and AE Processes started to become models-for more formal reasoning as Ada and Lori engaged in the model-eliciting activity.

\section{Evoking EA Process}

In what follows, I describe the students' situational activity for the EA Process model by engaging them in reinventing a definition of Eventually Constant Sequence. This will include describing how Ada and Lori created and used a concept image of Eventually Constant to create and refine a concept definition. I will present each of the proposed definitions with an analysis of the definition and the activity that led to it. Then, I will close this section with a discussion the EA Process model. I argue that after Ada and Lori defined Eventually Constant, the EA Process was a model-of Ada and Lori's defining activity. A table of Ada and Lori's proposed Eventually Constant Sequence definitions are provided at the end of the episode (see Figure 20). 
Creating concept image of Eventually Constant Sequence. The third session of the design experiment began with Ada and Lori engaging in a task that was designed to lead them to generate two sequences, one of which held the property that the terms were equal after the fourth step in the sequence. Specifically, I tasked the students to apply their root-approximation method, and specifically what we referred to as their bisection method, from our Introduction to Proof class to a function to which they hadn't yet applied it. The function was discontinuous and, in fact, did not have a root (see Figure 16A). The task assigned $a_{1}$ to 0 (an input value that mapped to a negative value) and $b_{1}$ to 1 (an input value that mapped to a positive value). To determine the next terms in the sequences, Lori tested whether the midpoint between $a_{1}$ and $b_{1}$ mapped to a positive or negative value. Since, the output of the midpoint between $a_{1}$ and $b_{1}$ was negative, she assigned $a_{2}$ to $1 / 2$ (the value of the midpoint between $a_{1}$ and $b_{1}$ ) and let $b_{2}=b_{1}$. Repeating this process, she found that the midpoint between $a_{2}$ and $b_{2}$ mapped to a negative value and so she defined $a_{3}=3 / 4$ (the midpoint between $a_{2}$ and $b_{2}$ ) and $b_{3}=$ $b_{2}$. In the same way, she defined $a_{4}=7 / 8$ and $b_{4}=b_{3}$. However, the midpoint of $a_{4}$ and $b_{4}$ mapped to a negative value and so she defined $a_{5}=a_{4}$ and $b_{5}=15 / 16$ (the midpoint between $a_{4}$ and $b_{4}$ ). They found one more step in the sequences, and then Ada observed that "it would kind of just stays like that forever," labeling " $a_{5}, a_{6}, a_{7}$, etc." with $a_{4}$. See Figure 16B. 


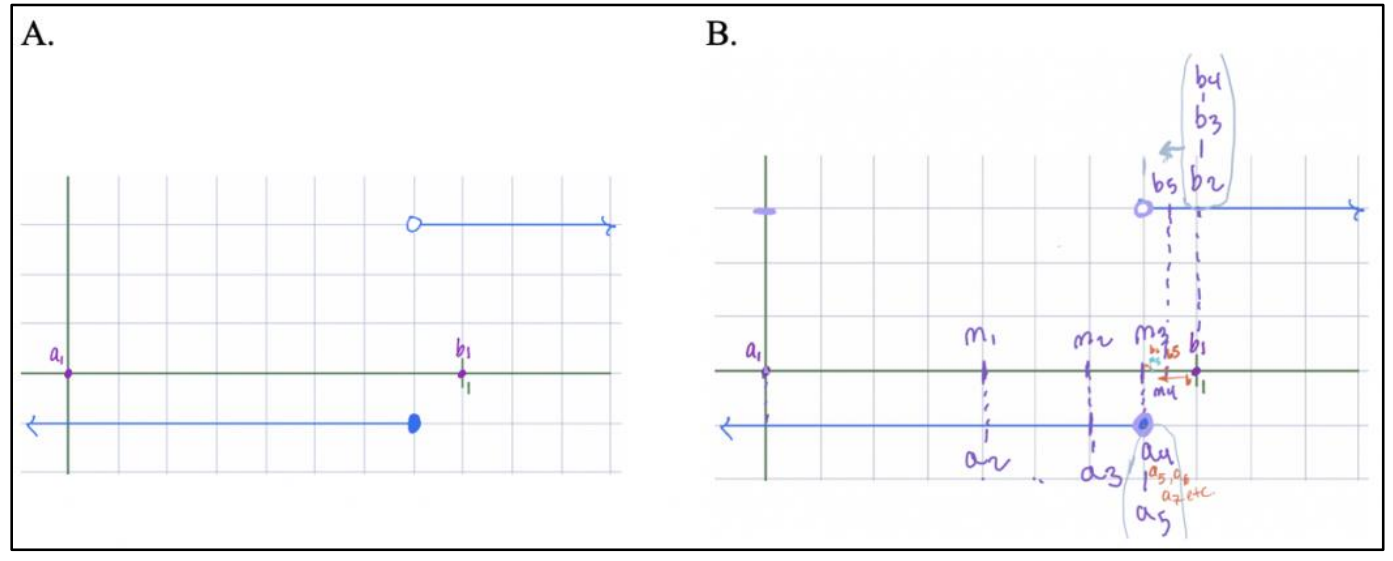

Figure 16. Generation of Eventually Constant Sequence.

As an attempt to focus their attention on the property that the terms of $\left\{a_{n}\right\}$ were equal after some step in the sequence, I asked Ada and Lori if both of the sequences converged and if so why. They conjectured that $\left\{b_{n}\right\}$ would converge because it was decreasing and bounded (an idea that was discussed in their prior course) and $\left\{a_{n}\right\}$ would converge because it was increasing and bounded, but it also held an additional property. Lori explained that "it reaches its bound" (meaning least upper bound). Notice that Ada and Lori began engaging in situational activity for the EA Process model during this task by beginning to create a concept image of Eventually Constant Sequences, which is a specific context that evoked an EA Process.

In Ada and Lori's Introduction to Proof class, it was normative to define new ideas when they arose in class. Seemingly recognizing this idea as a new concept, Ada explained, "Oh man, we need another definition!” This motivated Ada and Lori to define a new kind of sequence, which Lori proposed calling "Eventually Constant Sequence". In response, I suggested that Ada and Lori create examples and non-examples of Eventually Constant Sequences so that they could use them to write a definition. Ada and Lori continued to develop their concept image deciding that an Eventually Constant Sequence 
did not need to be monotone and provided an example (see Figure 17A), explaining that "it's all wack here (referring to the terms that are circled) but as soon as you hit this one here (referring to the eighth term) it is all constant." They also discussed other types of sequences, deeming the sequences depicted in Figure 17A-D as Eventually Constant Sequence and the sequences depicted in Figure 17E-G as non-examples of Eventually Constant Sequence. Although the sequences sketched in Figure 17A and Figure 17G look similar, Ada and Lori intended to capture two ideas: (1) the sequence in Figure 17A illustrated a sequence for which $a_{k}=0$ for all $k \geq 8$, and (2) the sequence in Figure $17 \mathrm{G}$ was a sequence that converge to 0 , but no term in the sequence equaled 0 .

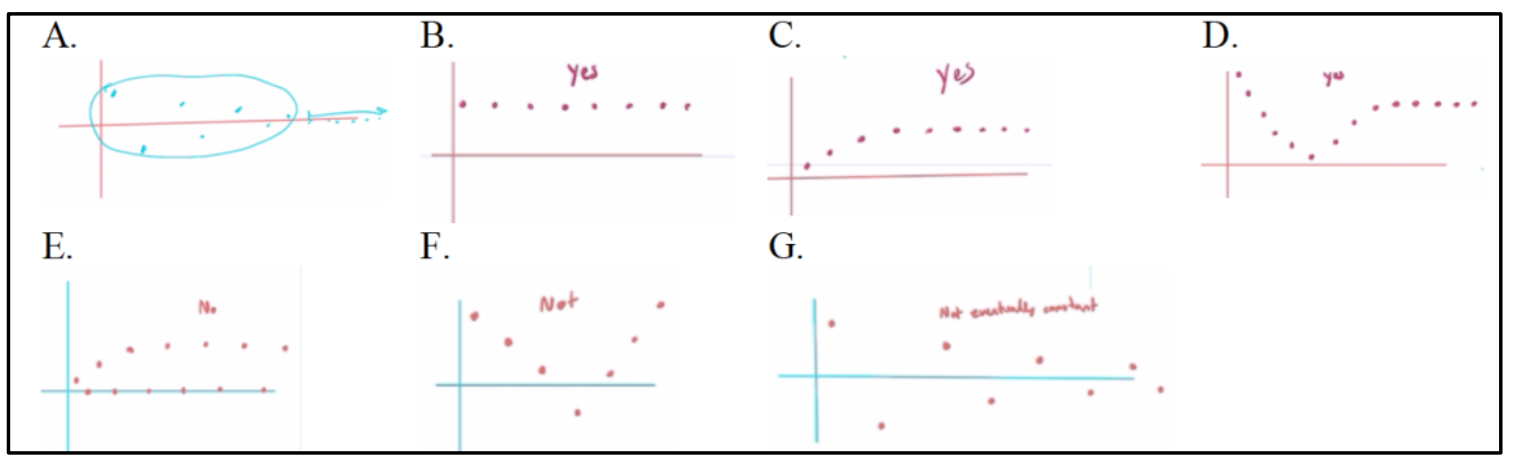

Figure 17. Examples and non-examples of Eventually Constant Sequence.

Once they were satisfied with their collection of examples and non-examples, Ada suggested that they had a shared concept image, explaining:

"I think it is important to pull up all these ideas because it allows us to like 'ok, well we all agree.' We don't really know what our definition is but we know what it looks like and we know what it doesn't look like. That's why I think it is important to have multiple cases to look at - just so that we can't put it in words yet but we know what we are looking for."

I suggest that there are a couple of features of the defining task that contributed to Ada and Lori developing a shared concept image of Eventually Constant Sequence. First, 
as Ada suggested, it was important that the students generated sequences and negotiated whether they were examples or non-examples of the concept. This allowed them to "know what [they were] looking for", as Ada put it. Secondly, the concept of Eventually Constant Sequence naturally came from applying their bisection method (a method that they previously reinvented to approximate a root of a polynomial) to the function in Figure 16A because the sequence of left endpoints was increasing and attained its least upper bound. The shared experience allowed them to notice and find value in a sequence attaining its limiting value, which initiated their concept image of Eventually Constant Sequence.

After Ada's comment, I was confident that they agreed on what Eventually Constant Sequences looked like and so I tasked them: "Try to write down your idea in one sentence and it's ok if it doesn't work out perfectly, we can keep refining it." With this, Lori offered her first attempt:

Eventually Constant Sequence Definition A: If $k$ is very large, $a_{k}$ will equal $a_{k+1}$.

There are several important aspects to note about Eventually Constant Sequence Definition A. First, the statement seems to fit their concept image of Eventually Constant Sequence. However, the statement is ambiguous because the phrase ' $k$ is very large' leaves what counts as 'very large' up to interpretation. Second, it is likely that Ada and Lori were drawing on their prior experience of defining monotone sequences to come up with this statement since their monotone sequence definition used successive terms.

Addressing ambiguity in ' $\boldsymbol{k}$ is very large'. Ada expressed that she liked Lori's proposed definition (Definition A) and explained, "that's kind of the same thing as 'if $k$ is 
very large' so like - so the beginning of the sequence is beneath that largeness it does whatever it wants and then after it's reached the large portion of its life it will end constant." Notice that Ada used her concept image of Eventually Constant to evaluate the proposed definition; to her, the definition captured the idea that at some point the sequence will "end constant". Additionally, Ada's words "beneath that largeness" and "after it's reached the large portion of its life" treated the statement like there is a single point to be underneath. Lori appeared to recognize an opportunity to improve their definition to articulate their thinking by highlighting the particular point. She refined the definition to include the existence of some number $L$ :

Eventually Constant Sequence Definition B: For a sequence $a_{k}$, there exist some number $L$ beyond which $a_{k}=a_{k+1}$.

While the introduction of some number $L$ seems to address the issue of someone having to decide what is meant by ' $\mathrm{k}$ is very large', Eventually Constant Sequence Definition B is still ambiguous. In particular, as written the statement does not specify $L$ to be an index value, a sequence value, or some number (natural or real) to compare either index values or sequence values to. In what follows, Lori pointed to this uncertainty motivating the next refinement.

Addressing ambiguity by specifying $L$. Lori turned to one of the Eventually Constant Sequence examples and said: "So this way... it can do all sorts of wacky stuff but there is some $L$ (marking an $L$ on an example, see Figure 18), and I don't know if it makes sense to have it a natural number or a member of the sequence..." I redirected the question of if $L$ was a natural number or a member of the sequence to Ada and she 
answered, "I think it should be a natural number that way $L$ isn't unique to any particular sequence. Because if you said [...] $L$ is like 8, and then you're like I am on this sequence and there isn't any 8 in there. Like you couldn't relate sequences..."

I take Ada's comment to mean that she preferred that $L$ to be a natural number because every sequence has inputs that are the set of natural numbers which enables them to relate $L$ to any sequence. Alternatively, if $L$ was considered to be a member of the sequence (e.g., $a_{k}=8$ ), then there was no guarantee that the particular real number was an output of every Eventually Constant Sequence. Ada's preference prompted Lori to change "there exist some number $L$ " to read "there exist some natural number $L$ ":

Eventually Constant Sequence Definition C: For a sequence $a_{k}$, there exist some natural number $L$ beyond which $a_{k}=a_{k+1}$.

This statement seems to capture the defining property relatively well. However, it requires an interpreter to take 'there exist some natural number $L$ beyond which $a_{k}=$ $a_{k+1}$ ' to mean that the sequence terms are equal for every sequence step greater than $L$; 'beyond which' acts to quantify $k$ which is typically done by writing as 'for all $k \geq L$ '. Ada and Lori's next refinement led them to use this conventional language.

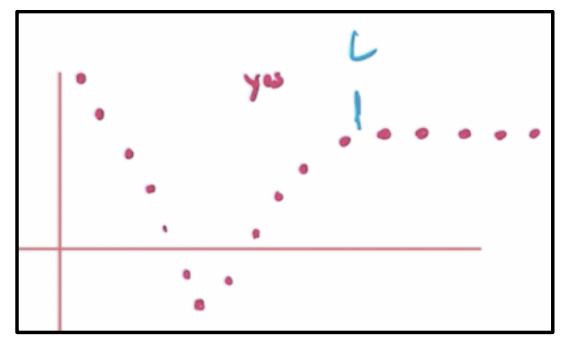

Figure 18. The location of L with an Eventually Constant Sequence

Using 'for all $\boldsymbol{k} \geq \boldsymbol{L}$ '. Immediately after Ada defined $L$ to be a natural number, Lori wanted to make it more explicit how $L$ related to the sequence. She asked Ada: "So 
do you want to include something about after $k$ is equal to $L$ like some way to connect $L$ to the sequence? (emphasis added)." Ada agreed and Lori replaced 'beyond which $a_{k}=$ $a_{k+1}$ ' with 'where for $k \geq L, a_{k}=a_{k+1}$ '.

This alteration makes it more obvious that a quantifier for $k$ is needed; one could interpret 'for $k \geq L$ ' as 'for some $k \geq L$ ' just as easily as 'for all $k \geq L$ '. Ada and Lori seemed satisfied and so they turned to their collection of examples and non-examples to evaluate it. During this, Lori changed one of their non-examples to the sequence depicted in Figure $17 \mathrm{~F}$ and they had the following exchange:

Lori: There is something that I would like to do to quickly change the sequence (referring to sequence in Figure 17F) to keep it so that it is not [Eventually Constant Sequence] so if we make it 'momentarily constant' let's make sure... it (meaning their definition) still accounts for that (altering Figure $17 \mathrm{~F}$ to

Figure 19A). So it seems like this is $L$ (labeling $L$, see

Figure 19B) and it fits here (labeling the fourth term with $a_{k}$ ) and this $a_{k}$ is equal to this $a_{k+1}$ (labeling the fifth term with $a_{k+1}$ ) and fits here (labeling the sixth term with $a_{k+2}$ ) but it does not fit here - beyond here. So, does our definition account for that? Ada: I think it does. I think you can also put like 'where for all $k \geq L$ ' because it might be misinterpreted as 'for some $k \geq L$ '. So, we might want to put an 'all' in there so that we are very clear that everything beyond.

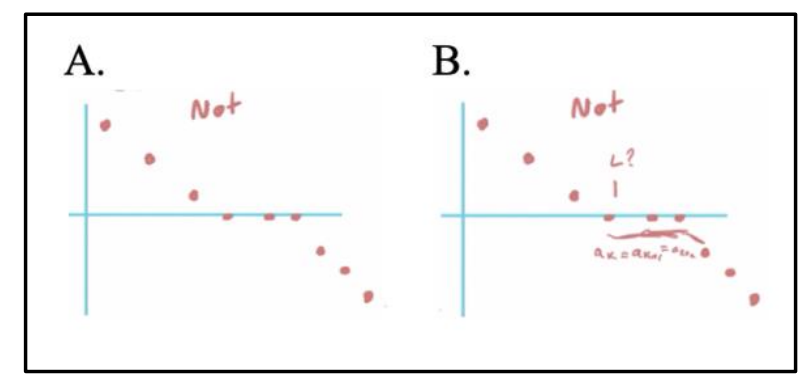

Figure 19. A "momentarily constant" sequence.

This was an important exchange between Ada and Lori. First, Lori introduced this non-example to seemingly test a type of sequence that they had not yet captured in their 
collection of (non-)examples given in Figure 17, specifically, a sequence that had some equal consecutive terms but was not an Eventually Constant Sequence. This non-example of Eventually Constant Sequence supported Ada to see a possible interpretation of their statement that they did not mean to communicate: one could interpret 'for $k \geq L$ ' as 'for some $k \geq L^{\prime}$. Ada added a universal quantifier to the variable $k$ to make sure that one could not misinterpret their definition that way:

Eventually Constant Sequence Definition D: A sequence is Eventually Constant if: For a sequence $a_{k}$, there exists some natural number $L$, where for all $k \geq L, a_{k}=a_{k+1}$.

I identify only minor issues with Eventually Constant Sequence Definition D. For instance, the introduction to the definition seems to be a bit clumsy due to the redundancy in 'A sequence is Eventually Constant Sequence if: For a sequence $a_{k} \ldots$ ' Ada and Lori addressed this in the next, and final, refinement. Despite this minor stylistic issue, the concept definition seemed to fit Ada and Lori's concept image.

Removing redundancy in the definition's introduction. The last refinement occurred after I asked Ada and Lori to reread their definition, which led Lori to recognize the redundancy in the definition's introduction. Lori suggested "trimming it down" and they altered it like by crossing out the "For a sequence $a_{k}$ ", changing it to the following: Eventually Constant Sequence Definition E: A sequence $a_{k}$ is Eventually Constant if there exists some natural number $L$, where for all $k \geq L, a_{k}=a_{k+1}$.

Discussion of evoking the EA Process. See Figure 20 for a summary of Ada and Lori's definition refinements for Eventually Constant Sequence. During this episode, Ada and Lori engaged in situational activity that was contextualized in creating a concept 
image of Eventually Constant Sequence and then used their concept image to create and refine their concept definition. Their activity involved mathematizing the concept of Eventually Constant Sequence that emerged from their exploration of applying their bisection method to a particular discontinuous function. They introduced an existentially quantified variable (i.e. there exists some natural number $L$ ) in order to indicate that there was a particular index value, $L$, such that all the terms associated with indices greater than $L$ mapped to the same value. They introduced a universally quantified variable (i.e. for all $k \geq L$ ) to clarify that their definition would exclude "momentarily constant" sequences. The collection of examples and the set of definition refinements began the chain of signification for EA Processes; each inscription took on the EA Process of the prior inscription (see Appendix). Notice that Ada and Lori's inscriptions evolved into an EA statement. At this point in the experiment, EA Processes were a model-of Ada and Lori's defining activity. That is, as the researcher, I could see that Ada and Lori were engaged in describing an EA Process in which a natural number $L^{*}$ could be selected so that $a_{k}=a_{k+1}$ for every $k \geq L^{*}$. But it did not appear as though Ada and Lori were yet aware that such a process existed outside of the concept of Eventually Constant Sequence.

\begin{tabular}{ll}
\hline Eventually & If $k$ is very large, $a_{k}$ will equal $a_{k+1}$ \\
Constant & \\
Sequence & \\
Definition A & \\
Eventually & For a sequence $a_{k}$, there exist some number $L$ beyond which \\
Constant & $a_{k}=a_{k+1}$ \\
Sequence & \\
Definition B &
\end{tabular}




$\begin{array}{ll}\begin{array}{l}\text { Eventually } \\ \text { Constant } \\ \text { Sequence } \\ \text { Definition C }\end{array} & \begin{array}{l}\text { For a sequence } a_{k}, \text { there exist some natural number } L \text { beyond } \\ \text { which } a_{k}=a_{k+1}\end{array} \\ \begin{array}{l}\text { Eventually } \\ \text { Constant }\end{array} & \begin{array}{l}\text { A sequence is Eventually Constant if: For a sequence } a_{k}, \text { there } \\ \text { exists some natural number } L, \text { where for all } k \geq L, a_{k}=a_{k+1}\end{array} \\ \begin{array}{l}\text { Sequence } \\ \text { Definition D }\end{array} & \text { A sequence } a_{k} \text { is Eventually Constant Sequence if there exists } \\ \text { Eventually } & \text { some natural number } L, \text { where for all } k \geq L, a_{k}=a_{k+1} \\ \text { Constant } & \\ \text { Sequence } & \end{array}$

Figure 20. A table of definition refinements for Eventually Constant Sequence.

\section{Evoking AE Process and further development of EA Process}

In the next section, I describe the students' situational activity for the AE Process model by engaging them in reinventing a definition of Prime Sequence. Again, I will begin with discussing the creation of their concept image and then describe the activity that led to each of their proposed concept definitions and an analysis of the definition. As the students engaged in situational activity for the AE Process, Ada and Lori engaged in more general activity (either referential or general activity) for the EA Process. I will conclude the section with a discussion about the EA and AE Processes models. I argue that (1) EA Processes became more general for Ada and Lori, (2) Ada and Lori's defining activity for Prime sequence was a model-of AE Processes, and (3) EA Process was a contrasting concept that supported the AE Process model. A list of Ada and Lori's proposed definitions for Prime sequence is given at the end of the episode in Figure 25.

Creating concept image of Prime Sequence. During the ninth session of the design experiment, I tasked Ada and Lori to define a new type of sequence which I called 
"Prime Sequence". I explained that a prime sequence contained all the prime numbers. Ada and Lori began engaging in situational activity by creating a concept image of Prime Sequence. They did so by negotiating what counted as an example and what counted as a non-example. They established that a prime sequence: (1) could contain numbers that were not prime, (2) could have the same prime number listed more than once, (3) did not have to necessarily list the prime numbers in order, (4) was not necessarily increasing, and (5) could never be a constant sequence. They produced sketches of five examples, including $a_{k}=k$ (see Figure $21 \mathrm{~F}$ ), several non-monotone examples (see Figure 21B, D, E), and the sequence that listed only the prime numbers in order (see Figure 21C). They also produced sketches of two non-examples, including $a_{k}=k^{2}$ (see Figure 21A) and $a_{k}=3$ (see Figure 21G). After they produced this collection of examples and nonexamples, I suggested that they try writing down a definition. Ada gave her first attempt: Prime Sequence Definition A: A prime sequence is a sequence that must contain the set of prime numbers in its range.

Ada and Lori seemed satisfied that their definition could sort their sequences into examples and non-examples. Notice Prime Sequence Definition A is an informal description and essentially the description that I gave Ada and Lori when I originally posed the task. 


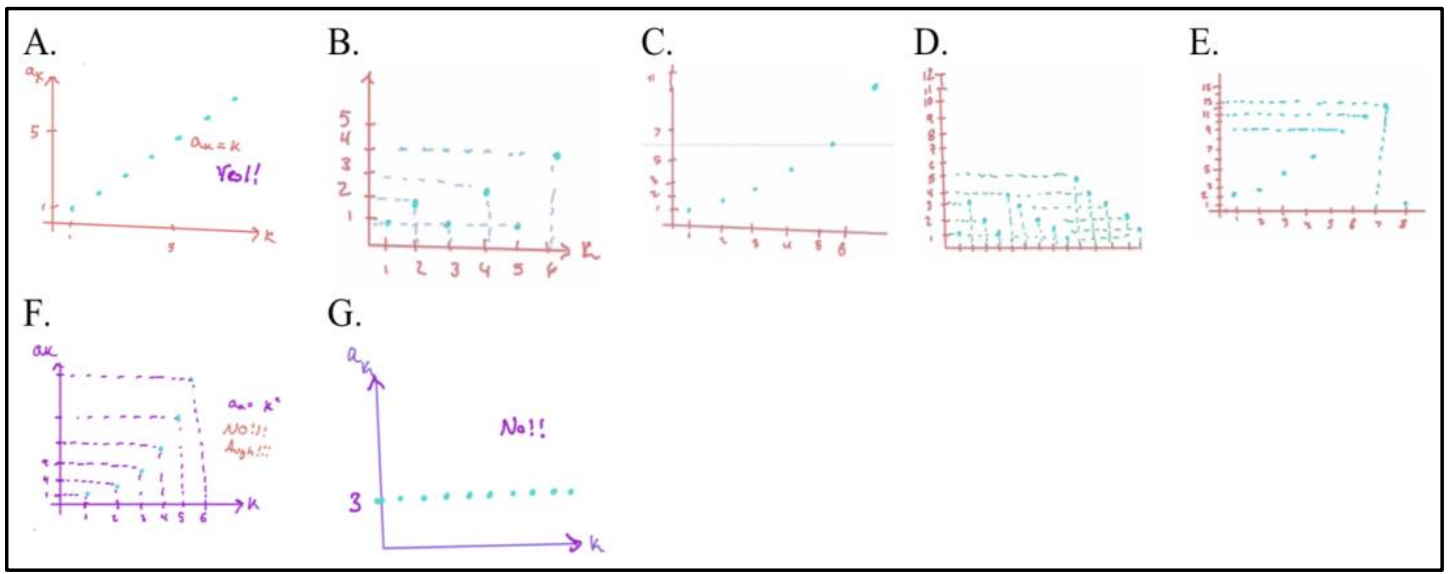

Figure 21. Examples and non-examples of Prime sequence.

Using 'for all' and 'there exists' in definition. Next, I reminded them that their previous definitions used the phrases 'for all' and/or 'there exists' and encouraged them to rewrite their definition using the phrases. With this the students continued to engage in situational activity and used their concept image to refine their definition. Ada commented, 'I feel like I definitely [want to] put the 'for all' with the prime numbers like 'for all prime numbers' or something like that." After some discussion about the set of prime numbers (which they denoted with $\mathbb{P}$ ), Lori wrote, 'For all prime numbers in $\mathbb{P}^{\text {' }}$ and then posed a question: "Where do we go from that? Like what do we put before and after?" which led to the following exchange:

Ada: That's a good question.

Lori: And if we want to have a 'there exists' we should probably...

Ada: There exists a natural number for all prime numbers. Something like that. Like you could just draw that connection.

Lori: Maybe a... (she starts writing)

Ada: Basically, saying that each prime number has its own natural number. Something like that.

Lori: Oh, I think I get it - hang on - so like there exists a $k$ an input $k$ which maps to... Ada: Yeah.

Lori: A prime number for all.

Ada: Mhm - there is like one prime - there's one natural number for all prime numbers. 
Notice that Ada seemed to clarify what she meant by "There exists a natural number for all prime numbers" when she said "Basically saying that each prime number has its own natural number". Although "There exists a natural number for all prime numbers" first uses an existential quantified variable (i.e. there exists a natural number) and is followed by a universal quantifier variable (i.e. for all prime numbers), Ada did not describe an EA Process. Rather she explained that she wanted the definition to convey that each prime is associated with one natural number. During this interaction, Lori produced the following statement:

Prime Sequence Definition B: There exists an input $k$ which maps to a prime number for all prime numbers in $\mathbb{P}$.

Although Prime Sequence Definition B is an EA statement, I do not think that Ada and Lori intended to represent an EA Process. I interpret Prime Sequence Definition $\mathrm{B}$ along with their interactions to mean that there is an index value where a given prime number shows up. Then, by adding 'for all prime numbers in $\mathbb{P}$ ', they suggested that this has to be the case for every prime number. However, one could easily interpret the statement differently than Ada and Lori intended since EA statements encode an EA Process. Using conventions, a reader might interpret Prime Sequence Definition B as there is an input $k^{*}$ such that $a_{k^{*}}=p$ for every prime number $p$. This was not Ada and Lori's intended meaning, nor does it describe a sequence since it suggests that there is an index value that lists every prime number. 


\section{Clarifying that more than one input value could map to the same prime}

number. Quickly after Lori updated their definition to Prime Sequence Definition B, Ada requested: "Could you say 'at least one input for each $k$ ' because that implies that there could be more than one input that would result in the same prime number but at least all of the prime numbers are accounted for once?" This resulted in Lori adding 'at least one' to the definition:

Prime Sequence Definition C: There exists at least one input $k$ which maps to a prime number for all prime numbers in $\mathbb{P}$.

Ada's request appears to be a move to make sure that the statement expressed Ada and Lori's intended meaning of the concept. In particular, Ada and Lori had agreed that a Prime Sequence could have the same prime number listed more than once (e.g., see the sequence depicted in Figure 6D). Adding 'at least one' seemed to be an attempt to clarify that more than one input value could map to the same prime number.

Note, that Prime Sequence Definition C still could suggest an EA Process. In the next session, Ada and Lori recognized this potential interpretation and clarified their intended meaning with their next refinement.

Addressing potential misinterpretation as an EA Process. After the refinement, Lori seemed concerned with the statement and so I asked her to explain her thinking. Lori explained, “... it's starting to sound like there's only one prime number in the whole set or in the whole sequence... because it is saying there exists at least one input which maps to a prime number (emphasizing 'a') and then it jumps into for all." I take this comment to mean that Lori thinks Prime Sequence Definition C could be 
interpreted as a type of sequence that contains exactly one prime number. Ada attempted to address her concern by erasing the phrase 'which maps to a prime number' so that it read, 'There exists at least one input $k$ for all prime numbers in $\mathbb{P}$ '. Immediately after Ada deleted the phrase, Lori read it out loud and then said, "Then that one $k$ outputs to all of them!" and they both laughed. After I probed them to explain their thinking, Lori suggested that 'There exists at least one input $k$ for all prime numbers in $\mathbb{P}$ ' seemed to imply that all there was an input $k$ which mapped to every prime number. In particular:

Lori: Okay our current problem is that we have this $k$ guy here he must - if this is our definition for a prime sequence then our prime sequence looks (draws sketch in Figure 22) $k$ is equal to one and two and three...

Ada: All on top

Lori: And that's not good.

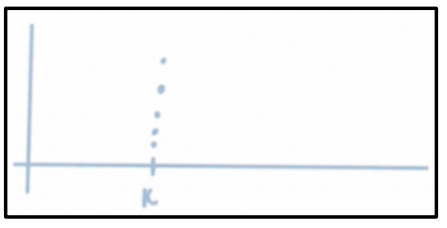

Figure 22. Issue with 'There exists at least one input $\mathrm{k}$ for all prime numbers in $\mathbb{P}$ '

Notice that here Ada and Lori describe an EA Process: there is one input $k$ that maps to all of the prime numbers. Ada and Lori using this EA Process to describe how their current definition for Prime Sequence failed to encode their concept image is evidence that the EA Process model is becoming more general for Ada and Lori. It is unclear if Lori referred to her experience with Eventually Constant Sequences to identify the EA Process, or if she recognized the process independent from their Eventually Constant defining activity. However, in either case, it is clear that she was thinking of the EA Process more generally than an idea that is specific to the Eventually Constant Sequence. 
Ada attempted to address their problem by using a different phrase, writing "for every prime number in $\mathbb{P} "$ next to Prime Sequence Definition D (See Figure 23), seemingly suggesting to replace the phrase 'for all' with 'for every'. She explained:

"I'm just trying to say that it isn't this scenario where they're all stacking on top of each other on accident like there's only one $\mathrm{k}$ for all of them. We're trying to say there's one for each of them... So that's what we're trying to say is that it's not that one k maps to all of them, it's that least all of them are accounted for once. And so, I felt like 'for every' could help with that."

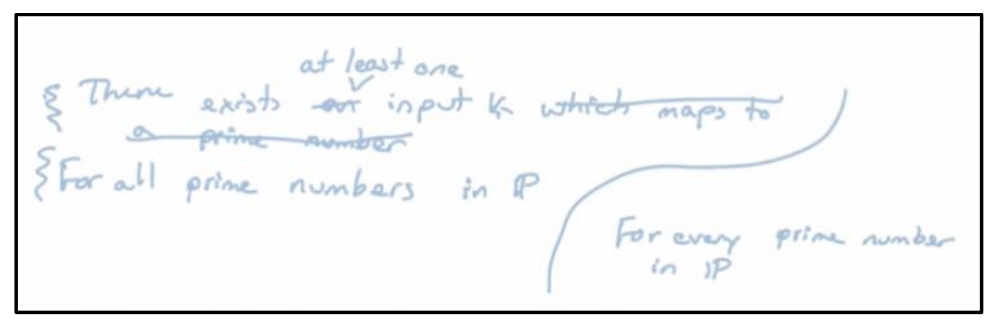

Figure 23. Ada's alternative phrase

While in the moment I did not follow-up with Ada for how 'for every' could help, Ada's previous description of the difference that she saw with different universal quantifiers is illuminating. In Chapter 2, I explored Ada and Lori's thinking about 'for every' and 'for all' and argued that they had a collective meaning of 'for all' whereas 'for every" "sounds more singular". With this interpretation in mind, it seems as though Ada was testing whether using 'for every prime number in $\mathbb{P}$ ' instead of 'for all prime numbers in $\mathbb{P}^{\prime}$ would fix the problem that there was one input $k$ that mapped to the whole set of prime numbers instead of one input that mapped to a singular prime number. However, she quickly disregarded this idea not seeing it as a solution to her problem, stating "it's ambiguous".

At this point, I saw what was written on their tablet as an opportunity to inspire them to rearrange their sentence. I asked, "So with this suggestion - 'for every prime 


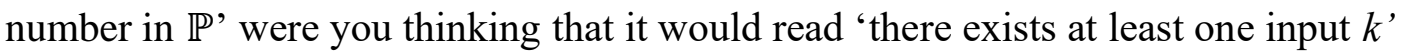
(pointing to the phrase then took a long pause) 'for every prime number in $\mathbb{P}$ ' (pointing to the phrase)?" With the gestures and long pause, I hoped that they might consider

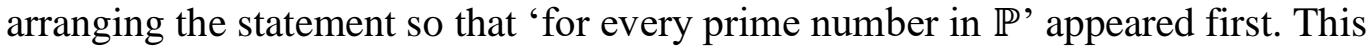
seemingly motivated Lori to suggest, "let's see what would happen if we swapped the order" and produced:

Prime Sequence Definition D: For all numbers in $\mathbb{P}$, there exists at least one input $k$ whose output is in $\mathbb{P}$.

Lori explained, "so we're saying that you've got all of your prime numbers and it's saying you will always be able to find at least one $k$ who will output to one of those prime numbers for all of the prime numbers" and then she continued:

It's for like for this guy (referencing the Prime Sequence in Figure 24). There's at least one $k$ which goes to three (marking the second tick mark on the horizontal axis with $k$ ). Sure, there are more, but there's at least one. And for every or for all or for every (laughs) prime number there is going to be some $k$ that goes up to it.

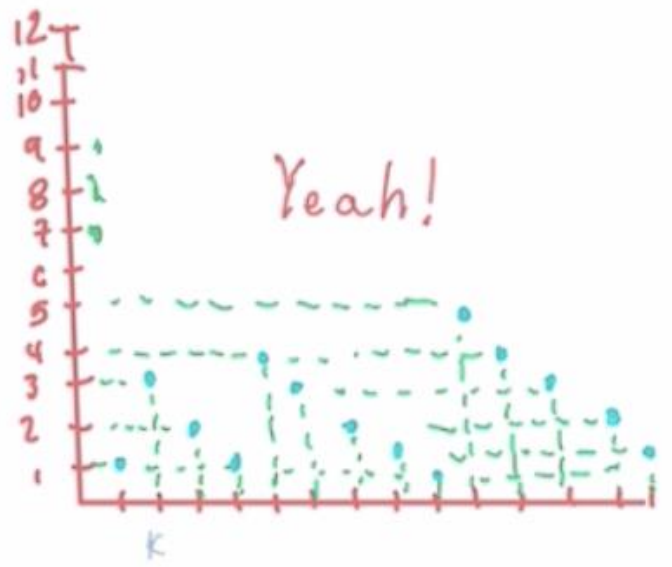

Figure 24. Example of Prime Sequence. 
I take Lori's description to be a description of an AE Process. If you consider a prime number (e.g., three), you can find at least one input that maps to the prime number. And given any prime number, "there is going to be some $k$ that goes up to it". Ada commented that she thought the refinement worked, saying "it's like the feng shui of syntax". Later she explained that the definition, "sound[s] more like this situation more like each of them get their own." Notice that Ada and Lori's situational activity for the AE Process was aided by the contrasting EA Process. In particular, the students aimed to describe the AE Process involved with Prime Sequence in a different way than Prime Sequence Definition $\mathrm{C}$ because that definition seemed to construe an EA Process, which was not the idea that Ada and Lori wanted to capture in their Prime Sequence definition.

Prime Sequence Definition D addressed the previous issue that the previous version of the statement could be interpreted as an EA Process. However, the mathematics community might have difficulty construing Ada and Lori's intended meaning because the definition does not use a variable in the phrase 'For all numbers in $\mathbb{P}$ ' and then does not use the variable in 'whose output is in $\mathbb{P}$ '. The statement seems ambiguous, and could led someone to interpret the statement as there is one prime number in the sequence.

Introducing the variable $\boldsymbol{p}$ and relation $\boldsymbol{a}_{\boldsymbol{k}}=\boldsymbol{p}$. Then, Ada focused on the first part of the statement (i.e. 'For all numbers in $\mathbb{P}$, there exists at least one input $k$ ') and explained that she preferred to use 'for every' instead of 'for all'. She said 'for all prime numbers in $\mathbb{P}$ there is one $k$, that's what we're saying, there is one $k$ for all prime numbers." I take Ada's comment to mean that it was important to use the phrase 'for 
every' instead of 'for all' because the 'for all' version of the statement suggests an EA Process. See Chapter 2.

Immediately after Ada's request to change 'for all' to 'for every,' Lori said “we kind of need more information now - who's this $k$ guy and what does he have to do with prime numbers?" This led her to quickly make their final refinement:

Prime Sequence Definition E: For every prime number $p$ in $\mathbb{P}$ there exists at least one input $k$ where $a_{k}=p$.

This definition is an AE statement that described the AE Process that Ada and Lori intended.

\section{Discussion of evoking AE Process and further development of EA Process.}

See Figure 25 for a summary of Ada and Lori's definition refinements for Prime Sequence. During the second episode, Ada and Lori engaged in situational activity by creating a concept image of Prime Sequence and then used their concept image to create a concept definition. The students described an AE Process contextualized in the task setting of defining Prime Sequence. Additionally, Ada and Lori's inscriptions related to Prime Sequences began the chain of signification for AE Processes where each inscription took on the AE Process meaning of the prior inscription (see Appendix). An AE Process was a model-of Ada and Lori's Prime defining activity because the defining activity involved describing an AE Process in which given any prime number, "there is going to be some $k$ that goes up to it". This process was contextualized in the task setting Prime Sequence and it did not appear that Ada and Lori were yet aware that the process existed outside of the Prime Sequence concept. Additionally, the EA Process became 
more general and more powerful during Ada and Lori's Prime defining activity because they recognized the phrase 'there exists at least one input $k$ for all prime numbers $p$ ' conveyed an EA Process, which did not reflect the process that they wanted to convey. The chain of signification for EA Processes illustrates that Ada and Lori recognized an EA Process in a different context than Eventually Constant Sequences (see Appendix). Additionally, the EA Process supported the AE Process model. In particular, the EA Process that Ada and Lori saw in 'there exists at least one input $k$ for all prime numbers p' was a contrasting concept that Ada and Lori used to describe the AE Process.

$\begin{array}{ll}\begin{array}{l}\text { Prime Sequence } \\ \text { Definition A }\end{array} & \begin{array}{l}\text { A prime sequence is a sequence that must contain the set of } \\ \text { prime numbers in its range }\end{array} \\ \begin{array}{l}\text { Prime Sequence } \\ \text { Definition B }\end{array} & \begin{array}{l}\text { There exists an input } k \text { which maps to a prime number for all } \\ \text { prime numbers in } \mathbb{P}\end{array} \\ \begin{array}{ll}\text { Prime Sequence } \\ \text { Definition C }\end{array} & \begin{array}{l}\text { There exists at least one input } k \text { which maps to a prime number } \\ \text { for all prime numbers in } \mathbb{P}\end{array} \\ \begin{array}{l}\text { Prime Sequence } \\ \text { Definition D }\end{array} & \begin{array}{l}\text { For all numbers in } \mathbb{P}, \text { there exists at least one input } k \text { whose } \\ \text { output is in } \mathbb{P}\end{array} \\ \begin{array}{l}\text { Prime Sequence } \\ \text { Definition E }\end{array} & \begin{array}{l}\text { For every prime number } p \text { in } \mathbb{P} \text { there exists at least one input } k \\ \text { where } a_{k}=p\end{array}\end{array}$

Figure 25. A table of definition refinements for Prime Sequence.

\section{Moving to more general activity}

In the following section, I describe Ada and Lori's activity during the model-

eliciting activity, in which the students were instructed to use their definitions to write an instruction manual for peers to use $\mathrm{AE}$ and EA statements when defining. During this 
task, Ada and Lori first engaged in referential activity for the EA and AE Processes models as they reflected on a set of definitions including their definitions of Eventually Constant Sequence and Prime Sequence. Then, their activity shifted to general activity when they started to write their instruction manual. I will conclude this section with a discussion of the evolution of the two models. I argue that the EA and AE Processes began the model-of/model-for transition.

The design goal of the eleventh (and last) session was to support Ada and Lori in recognizing the processes as a tool for more general mathematical activity and coordinating these relationships with the grammar of a statement. At the beginning of the session, I provided Ada and Lori the definitions that they constructed during Episodes 1 and 2 as well as an additional statement (see Figure 26). Notice there is a slight difference in Eventually Constant Sequence Definition E and the Eventually Constant Sequence definition in Figure 26: I replaced 'for all' with 'for every'. I made this change because Ada and Lori used two different phrases for the universal quantifier in their Eventually Constant Sequence Definition and Prime Sequence Definition ('for all' and 'for every', respectively) and so I thought that using the same phrase would allow them to contrast the sentence structures. Additionally, I chose 'for every' instead of 'for all' because 'for all' proved challenging for Ada and Lori to convey the AE Process in Prime Sequence since they tended to associate 'for all' with the whole set that the quantified variable held membership. Whereas, it seemed Ada and Lori could use 'for every' to describe either an $\mathrm{AE}$ and or EA Process. 
I constructed the third statement (which we referred to as Star) based on their previous written work. Specifically, I constructed the statement by altering one of their inscriptions during their defining activity for Prime Sequence that conveyed an EA Process for Ada and Lori (i.e. 'there exists at least one input $k$ for all prime numbers in P'). The altered statement that I gave them replaced 'for all' with 'for every', introduced the variable $p$ to denote a prime number, and added the conclusion to the end of the statement:

A sequence $\left\{a_{n}\right\}$ is $* * *$ if there exists at least one input $k$ for every prime number $p$ in $\mathbb{P}$ where $a_{k}=p$.

I constructed the statement to provide another contrasting case for Ada and Lori. Notice that the Star statement has a 'there exists... for every... where' structure rather than 'there exists... where... for every' structure (like the Eventually Constant Sequence Definition). This allowed me to test whether Ada and Lori were simply attending to the order of the quantifiers to interpret the statement or more carefully, attending to aspects of the full statement to understand the meaning.

A sequence $\left\{a_{n}\right\}$ is Eventually Constant Sequence if there exists some natural number $L$, where for every $k \geq L, a_{k}=a_{k+1}$.

A sequence $\left\{a_{n}\right\}$ is Prime if for every prime number $p$ in $\mathbb{P}$, there exists at least one input $k$ where $a_{k}=p$.

A sequence $\left\{a_{n}\right\}$ is $*_{* *}$ if there exists at least one input $k$ for every prime number $p$ in $\mathbb{P}$ where $a_{k}=p$.

Figure 26. Statements provided for Ada and Lori to aid them in writing instruction manual task. 
Ada and Lori began engaging in referential activity by reflecting on the statements and describing their meanings that were portrayed in the statements. They described Eventually Constant Sequence and Prime Sequence in a manner consistent with their previous descriptions of the concepts. However, they seemed troubled by the Star statement. Lori explained:

"So, it's because I can sort of think of it there's a $k$ for every $p$. So, like here's $p_{1}$ oh it has its little $k$ buddy there, and here's $p_{2}$ and oh he's got a $k_{2}$. He's so happy - he's got a $k$. (See Figure 27A) And that's nice because then you get like: $k_{1}, k_{2}, p_{1}, p_{2}$. Yay, they're happy! (See Figure 27B). But then I can read it again and see there is one $k$ for every prime number where $a_{k}=p$. And then it's back to this big ugly guy (See Figure 27C). So I think that means it's a bad definition."

Notice that Lori first described an AE Process that they decided was encoded in their Prime Sequence definition and then described an EA Process. Moreover, she thought that the Star statement is a "bad definition" because it can be interpreted either way. This suggests that Lori was attending to more than just the order of the quantifiers in order to interpret the statement.

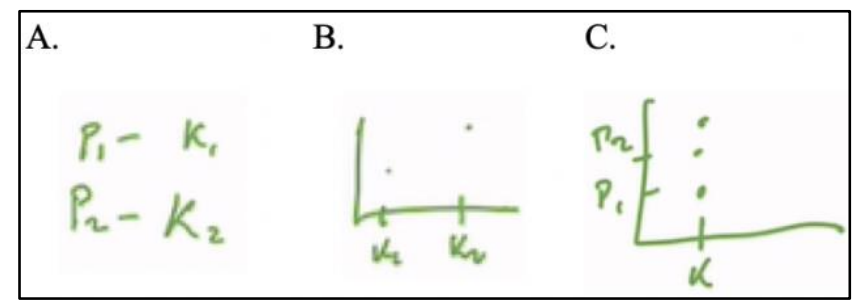

Figure 27. Lori's illustrations of Star interpretations

At this point, Ada and Lori focused on the phrase 'there exists at least one' in the Star statement, seemingly because 'at least one' did not appear in the Eventually Constant Sequence Definition but did in the other two statements. Additionally, Ada suspected that removing 'at least one' might make it clearer how one might interpret the Star statement. 
At this point, I wrote 'there exists $a$ ' and 'there exists at least one $a$ ' on their tablet and asked them if there was a difference between the two phrases. Ada then responded, "I would say if you tacked 'for every' on the end of each of those phrases I would say they're the same... I think it is the same interpretation but I don't like adding 'at least one' because I think it makes it muddier." Then, Lori changed the prompt so that it read, 'there exists $a$ for every $b$ in $B$ ' and 'there exists at least one $a$ for every $b$ in $B$ ' and then sketched accompanying diagrams (see Figure 28). She stated that the sketch in Figure 28A represented 'there exists $a$ for every $b$ in $B$ ', the sketch in Figure 28B represented 'there exists at least one $a$ for every $b$ in $B$ '.

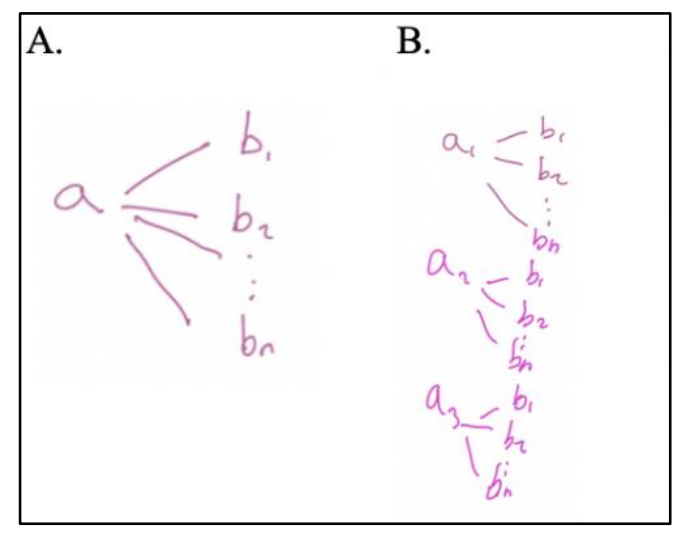

Figure 28. 'There exists a for every b in B' versus 'There exists at least one a for every b in B'

There are several important things to notice with the diagrams. First, both diagrams seem to depict EA Processes, indicating that Lori opted to interpret these sentence structures with EA Processes (despite the absence of 'such that' or 'where'). Second, both diagrams depict EA Processes without the Eventually Constant context, suggesting that the students were beginning to engage in general activity. Third, Lori was considering the difference in the two statements to be either (1) there was exactly one $a$ that corresponded to every $b$, or (2) there was one (but could be more than one) $a$ that 
corresponded to every $b$. At this point, Ada requested Lori to "explain it in terms of like the prime sequence.” As Lori produced the diagram in Figure 28, she explained:

"So, for the prime sequence you have $p$ - kind of on this side like $p_{1}$ - let's look at him now. We know that he has to have some $k, p_{2}$ we know he has to have a $k, p_{3}$ he has to have a $k$, but he can also have another $k, p_{4}$ he can have $k_{4}$, or something like that." (See Figure 28.)

In contrast to the EA Processes that Lori described with $a$ and $b$, Lori described an AE Process - taking $p_{1}$ and finding a $k_{1}$ presumably so that $a_{k_{1}}=p_{1}$, then take a $p_{2}$ and finding a $k_{2}$ presumably so that $a_{k_{2}}=p_{2}$, and so on. She also notes that you could find more than one index for a particular prime number (illustrated by $p_{3}$ paired with $k_{3}$ and $k_{5}$ ). Notice that in contrast to the diagram in Figure 28, Figure 29 illustrates an afterimage of the process described in an AE Process. Specifically, each of the prime numbers correspond with some index value(s). Lori was able to produce the diagram by engaging in referential activity, reflecting on their definition and the process that it represented.

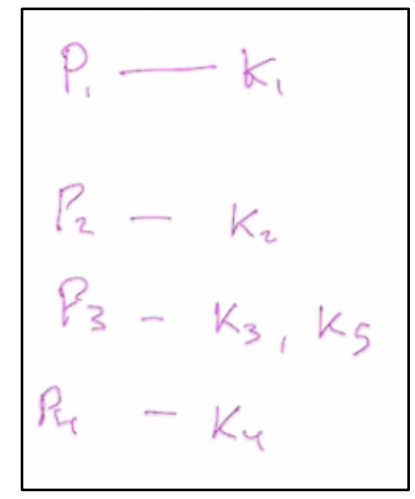

Figure 29. Illustration of Prime Sequence

At this point, Ada restated her preference that she wanted 'there exists' and 'there exists at least one' to be interpreted the same way. She seemingly imagined their Prime Sequence Definition without the phrase 'at least one' and explained, 'I don't think that 
simply the fact that it doesn't have 'at least one' it doesn't necessarily negate that there can be more than one input for each prime number." Lori clarified, "So you're saying that saying 'there exists' means there doesn't have to be just one?" Ada confirmed and then deleted 'at least one' from the Star Statement and Prime Sequence Definition. Although Ada and Lori did not mention this at the time, notice that this was in fact the interpretation that makes their Eventually Constant Sequence Definition work. In particular, if a sequence $\left\{a_{k}\right\}$ is Eventually Constant Sequence then there are infinitely many natural numbers $L$ such that $a_{k}=a_{k+1}$ for every $k \geq L$.

Then, the students began to engage in more general activity by Lori introducing an analogy to capture her thinking about EA and AE statements. She presented the picture in Figure 30 and started to explain, "I was focusing on what it's actually making you think when you hear 'for every' and then 'there exists'." And continues:

Lori: So, whenever you have a 'there exists' and [then] 'for every,' these three objects have to work for all of the options on the slider.

Teacher-researcher: Three?

Lori: That's just arbitrary. Yeah, these guys here (referring to the three objects under 'there exists') have to work and do their mojo for all of the options on the slider.

Ada: I kind of understand what you're saying.

Lori: It's like thinking of these single objects which you've just introduced they have to do whatever the definition is saying for every single option on the slider and that's what this ordering implies is that these work for everybody...

With this exchange, I think that Lori was trying to articulate that EA statements describe an EA Process. Specifically, for Lori, an EA statement described a situation in which you can find at least one object that must "do whatever the definition is saying for every single option on the slider". Notice that Lori described the analogy without the use 
of the Eventually Constant Sequence definition providing more evidence that she began to engage in general activity for the EA Process model.

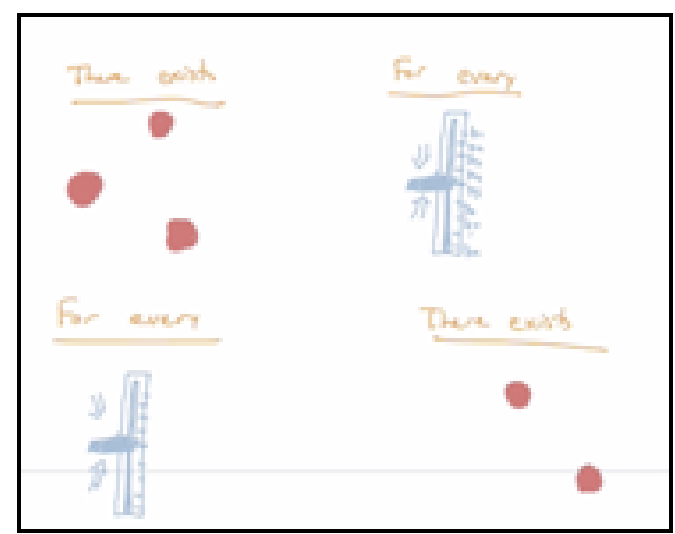

Figure 30. Lori's red objects-and-slider analogy

Lori continued to contrast this with AE statements, explaining: "But this ordering of 'for every' and then 'there exists' is a little bit freer and more like the stationary 'there exists' guys that we've defined they're less important and they're more like side tools that we can pick up and use." She then engages in referential activity for the AE Process model and refers to the Prime Sequence Definition and explains, "This goes 'for every' and then 'there exists' so it fits into this ordering where you can slide down all of the primes and you know there's going to be at least one of these that you can use for whatever space on the slider you know." I then confirmed with Lori that by 'one of these' she meant one of the $k$ values.

Here, Lori attempted to articulate that AE statements describe an AE Process. She first contrasted an $\mathrm{AE}$ statement with an EA statement, describing conveyed meaning of an AE statement as "freer". For Lori, an AE statement describes a situation in which you can slide down all of the values from the set that the universally quantified variable belongs to (e.g., the set of prime numbers) and know that there is going to be at least one 
member of the set that the existentially quantified variable belongs (e.g., at least one $k$ value) that can do whatever relation that definition describes for each location on the slider. Additionally, she explained that the variable quantified by 'there exists' ("the stationary 'there exists' guys") were less important in an AE statement than an EA statement. I interpret this as Lori highlighting that in an EA statement, you must find a one value from the set that the existentially quantified variable belongs to such that the definition is satisfied for every value in the set that the universally quantified variable belongs. Whereas in an AE statement, you can iteratively take a member of the set that the universally quantified variable belongs and pair it with a member of the set that the existentially quantified variable belongs that fits definition's relation.

At this point, I tasked Ada and Lori to write instructions intended for a peer. After several minutes, they produced the instructions and accompanying diagrams. See Figure 31. Note, their diagram that describes the relationship between $a$ and $b$ for an EA statement used alpha in place of $a$ and beta in place of $b$ for no other reason than "to make things fancy," according to Lori.

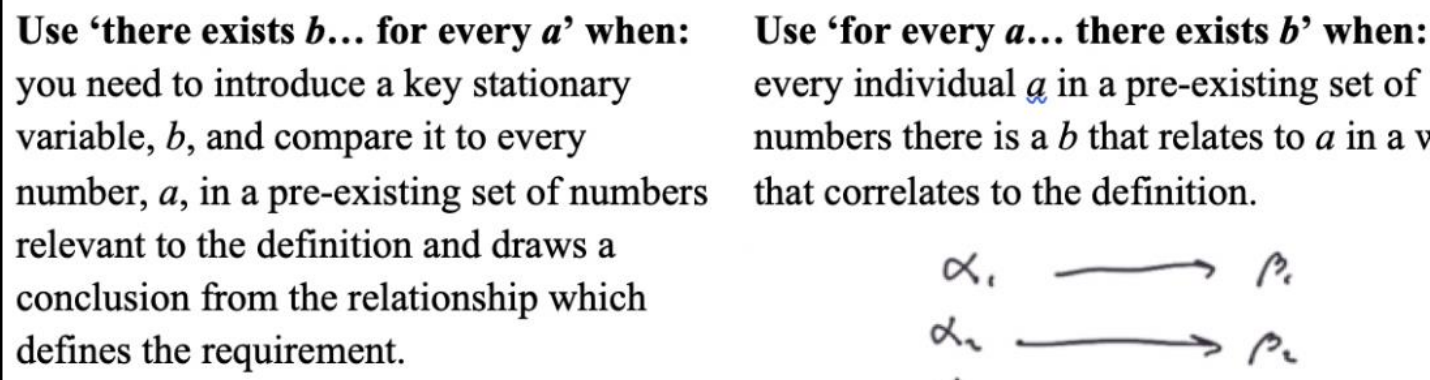
every individual $a$ in a pre-existing set of that correlates to the definition.

Use 'for every $a$... there exists $b$ ' when: for numbers there is a $b$ that relates to $a$ in a way 
Figure 31. Ada and Lori's instruction manual

I interpret Ada and Lori's instructions as describing two processes captured by EA and AE statements. Under the "Use 'there exists $b \ldots$ for every $a$ ' when:" prompt, they stated that there is one stationary value, $b$, that is compared to all potential values $a$ by attending to the conclusion's requirement. Their diagram illustrated an after picture of the process in an EA Process in which $b$ is associated with $a_{1}, a_{2}, a_{3}, \ldots, a_{n}, \ldots$. Under the "Use 'for every $a \ldots$ there exists $b$ ' when:", they explained that every $a$ must have a $b$ that relates to it in such a way that correlates to the definition. Their diagram depicted an after picture of the process in an AE Process: $\alpha_{1}$ is associated with $\beta_{1}, \alpha_{2}$ is associated with $\beta_{2}, \alpha_{3}$ is associated with $\beta_{3}, \alpha_{n}$ is associated with $\beta_{n}$, and so on. Note that the manual is more general and does not reference Eventually Constant Sequence or Prime Sequence.

Discussion of moving to more general activity. During this episode Ada and Lori engaged in referential activity in order to reflect on the given statements and discuss the processes that they represented. As they engaged in the task of writing an instruction manual for a peer to be able to use EA and AE statements when defining, their understandings of the EA and AE Processes in EA and AE statements became more general and more powerful. In particular, the instruction manual task supported Ada and Lori to be more explicit about EA and AE Processes evidenced through a variety of inscriptions. The changes are tracked in the two chains of signification given in the Appendix. In this episode, the inscription given in Figure 27 that represented the possible EA Process encoded in the Star statement evolved into the diagram of the EA Process between $a$ and $b$ in Figure 28, which evolved into the EA Process between the object and slider in Figure 30, and finally evolved into their instruction manual for using 'there 
exists $b \ldots$ for every $a \ldots$ ' (Figure 31 ). Additionally, the inscriptions in Figure 27 that represented the possible AE Process encoded of the Star Statement evolved into the diagram depicting the AE Process in the Prime Definition (Figure 29), which evolved into the AE Process between the object and slider in Figure 30, and then into the instruction manual for using 'for every $a \ldots$ there exists $b \ldots$ ' given in Figure 31 . The generality of Ada and Lori's instruction manual gives evidence of the beginning of the model-of/model-for transition for the two models. That the model-eliciting activity initiates a shift from referential to general activity by engaging students in writing an instruction manual for when to use EA and AE statements when defining. There was evidence that Ada and Lori both reasoned about the EA and AE Processes independent of Eventually Constant Sequence and Prime Sequence (e.g., with the instructional manual) and also folded back to referential activity to reflect on their defining activity (e.g., when Lori used the Prime Sequence definition to discuss her objects-and-slider analogy).

\section{Conclusion}

In Chapter 2, I offered a description of the dynamical processes that are encoded in EA and AE statements (which I refer to as EA and AE Processes, respectively). In this study, I offer a detailed description of Ada and Lori reinventing EA and AE Processes. I viewed the EA and AE Processes as two, co-emerging models. EA Processes are embedded in EA statements such as the students' final refinement of their Eventually Constant Sequence. The particular EA Process that the students in this study mathematized through their Eventually Constant Sequence defining activity is there is an index value $L^{*}$ that can be selected so that for every $k \geq L^{*}, a_{k}=a_{k+1}$. AE Processes are 
embedded in AE statements such as the students' final refinement of their Prime sequence. The particular AE Process that the students in this study mathematized through their Prime Sequence defining activity is that if you select a prime number $p_{1}$ then you can find a natural number $n_{1}$ such that $a_{n_{1}}=p_{1}$, and then if you select a prime number $p_{2}$ then you can find a natural number $n_{2}$ such that $a_{n_{2}}=p_{2}$, and this iterative pairing process can continue for every prime number. This study investigated the feasibility of students reinventing these processes by first engaging students in defining concepts that evoke EA and AE Processes.

This paper takes initial steps toward articulating a local instructional theory that frames the students' reinvention process in terms of the emergent models construct. The instructional theory first promotes engaging students in situational activity for the EA Process model by engaging students in reinventing a definition of a concept that is likely to evoke an EA Process for the students. In this study, I engaged students in reinventing Eventually Constant Sequence because this was a concept that emerged from their prior mathematical activity and I thought the students were likely to describe it as an EA Process.

The instructional theory then promotes engaging students in situational activity for the AE Process model by engaging students in reinventing a definition of a concept that is likely to evoke an AE Process for the students. In this study, I engaged students in defining Prime Sequence because the students had prior experience with classifying types of sequences, and I conjectured that the concept would evoke an AE Process for the students. As students engage in defining a concept that evokes an AE Process, the EA 
Process can be leveraged as a contrasting concept. For instance, in this study, the students recognized the wording of a proposed definition had the potential to convey an EA Process, which was not the process that they wanted to convey in their definition. In this way, the EA Process was a contrasting concept to an AE Process that supported the students in describing their meaning in a different way.

The instructional theory then promotes engaging students in referential activity by engaging students to reflect on the grammar of the resulting set of definitions in relation to the process that they describe. The instructional theory initiates a shift from referential to general activity by engaging students in writing an instruction manual for when to use EA and AE statements when defining. This task promotes students to recognize and begin to make use of the EA and AE Processes by requesting that they coordinate the grammar of the statements with the intended meaning. In this study, the students folded back to referential activity by using their definitions as examples of EA and AE Processes.

This study offers a case study of students reinventing the dynamical processes of EA and AE Processes that are encoded with statements with multiple quantifiers. Here I offer a proof of concept for an instructional approach in which students engaged in defining concepts and then reflected on the resulting set of definitions in order to learn about the dynamical processes.

\section{Discussion}

I attribute Ada and Lori's success in reinventing EA and AE Processes to the task design and the discourse among the students and myself. First, the task design involved 
making contexts meaningful for the students. This was accomplished in two ways. First, a process that the students previously reinvented evoked the idea of Eventually Constant Sequence. This allowed the concept to be especially tangible and relevant to the students. Second, students explored and identified a wide variety of (non-)examples of the concepts. This allowed the students to recognize nuances of the concepts and narrow in the underlying concepts. The task design leveraged the contexts by supporting the students in articulating their meanings. The students were encouraged to use various informal modes to conveying their meanings (using informal language, diagrams, etc.) and were progressively supported to encode their meanings using EA and AE statements. This design is in stark contrast to interpretation tasks in which students are expected to use unknown syntaxial rules to gain insight about the unknown mathematical meanings. The task design that I present here sets students up to succeed because it eliminates one of the unknowns, allowing students to leverage their mathematical meanings to learn about syntax.

The model eliciting activity was also key to the task design. I argue that the instruction manual task supported the model-of/model-for transitions for EA and AE Processes. Students will be better equipped to interpret statements with multiple quantifiers after this intervention because the relationships become more general and powerful for the students. Their instruction manual can also be viewed as a resource to 
support them in interpreting EA and AE statements because it should describe the processes that are encoded in each statement.

The implementation of the tasks was also an integral part of the success. To be clear, Ada and Lori did not reinvent the EA and AE Processes in AE and EA statements on their own. As the teacher-researcher, I played a significant part in guiding them in both defining the two concepts and supporting them in writing their instructional manual. In Chapter 4, I cast this role as a broker (Rasmussen et al., 2009) between the local community with the students and the broader mathematics community. In this study, I attempted to build on their thinking and inspire the students to consider restructuring their Prime Sequence definition. I also interpreted between communities by suggesting that they use 'for all' and/or 'there exists' in their Eventually Constant Sequence definition. The point of this approach is not to have students independently reinvent the EA and AE Processes; rather, it is to guide students to reinvent these ideas in a way that allows students to take ownership over the mathematics created.

As a researcher that subscribes to the theory of Realistic Mathematics Education, I think that mathematics should be learned by creating meanings, and gaining fluency with statements with multiple quantifiers, or even more generally mathematical language, is no exception. This paper illustrates that students can be supported to make progress on this fluency by engaging in defining. This study not only contributes to the research literature by offering a more authentic way to learn the mathematical language, but it also is an initial contribution to re-envisioning proof-oriented instruction. 


\section{References}

Carlson, M., Larsen, S., \& Lesh, R. (2003). Integrating a models and modeling perspective with existing research and practice. Beyond Constructivism: Models and Modeling Perspectives on Mathematics Problem Solving, Learning, and Teaching, $465-478$.

Cobb, P., \& Gravemeijer, K. (2008). Experimenting to support and understand learning processes. Handbook of design research methods in education: Innovations in science, technology, engineering, and mathematics learning and teaching, 68-95.

Cobb, P., Gravemeijer, K., Yackel, E., McClain, K., \& Whitenack, J. (1997).

Mathematizing and symbolizing: The emergence of chains of signification in one first-grade classroom. Situated cognition: Social, semiotic, and psychological perspectives, 151-233.

Dawkins, P. C., \& Roh, K. H. (2016). Promoting metalinguistic and metamathematical reasoning in proof-oriented mathematics courses: A method and a framework. International Journal of Research in Undergraduate Mathematics Education, 2(2), $197-222$.

Dawkins, P. C., \& Roh, K. H. (2019). Assessing the Influence of Syntax, Semantics, and Pragmatics in Student Interpretation of Multiply Quantified Statements in Mathematics. International Journal of Research in Undergraduate Mathematics Education, 1-22. 
David, E. J., Hah Roh, K., \& Sellers, M. E. (2020). Teaching the representations of concepts in Calculus: The case of the intermediate value theorem. Problems, Resources, and Issues in Mathematics Undergraduate Studies, 30(2), 191-210.

Dubinsky, E., \& Yiparaki, O. (2000). On student understanding of AE and EA quantification. Research in Collegiate Mathematics IV, 239-289.

Durand-Guerrier, V. (2003). Which notion of implication is the right one? From logical considerations to a didactic perspective. Educational Studies in Mathematics, 53(1), 5-34.

Durand-Guerrier, V., \& Arsac, G. (2005). An epistemological and didactic study of a specific calculus reasoning rule. Educational Studies in Mathematics, 60(2), 149-172.

Durand-Guerrier, V., Boero, P., Douek, N., Epp, S. S., \& Tanguay, D. (2011). Examining the role of logic in teaching proof. In Proof and proving in mathematics education (pp. 369-389). Springer, Dordrecht.

Epp, S. S. (2009). Proof issues with existential quantification. Proceedings of the ICMI Study 19 Conference: Proof and Proving in Mathematics Education, 1, 154-159.

Gravemeijer, K. (1998). Developmental research as a research method. In Mathematics education as a research domain: A search for identity (pp. 277-295). Springer.

Gravemeijer, K. (1999). How emergent models may foster the constitution of formal mathematics. Mathematical Thinking and Learning, 1(2), 155-177.

Gravemeijer, K., \& Doorman, M. (1999). Context problems in Realistic Mathematics Education: A Calculus Course as an example. Educational Studies in Mathematics, 39(1-3), 111-129. 
Gravemeijer, K., \& Terwel, J. (2000). Hans Freudenthal: A mathematician on didactics and curriculum theory. Journal of Curriculum Studies, 32(6), 777-796.

Freudenthal, H. (1973). De niveaus in het leerproces en de heterogene leergroep met het oog op de middenschool. In Gesamtschule conferentie (pp. 88-98).

Larsen, S. (2004). Progressive Mathematization in Elementary Group Theory: Students Develop Formal Notions of Group Isomorphism (Doctoral dissertation, Arizona State University, Tempe, United States). Retrieved from https://math.la.asu.edu/ carlson/Dissertations/Sean\%20Larsen\%20Dissertation.pdf

Larsen, S. (2013). A local instructional theory for the guided reinvention of the group and isomorphism concepts. The Journal of Mathematical Behavior, 32(4), 712-725. https://doi.org/10.1016/j.jmathb.2013.04.006

Larsen, S., \& Lockwood, E. (2013). A local instructional theory for the guided reinvention of the quotient group concept. The Journal of Mathematical Behavior, 32(4), 726-742. https://doi.org/10.1016/j.jmathb.2013.02.010

Lesh, R., Hoover, M., Hole, B., Kelly, A., \& Post, T. R. (2000). Principles for developing thought-revealing activities for students and teachers. In Research design in mathematics and science education (pp. 591-646). Lawrence Erlbaum Associates, Inc..

Roh, K. H. (2010). An empirical study of students' understanding of a logical structure in the definition of limit via the $\varepsilon$-strip activity. Educational Studies in Mathematics, 73(3), 263-279. 
Roh, K. H., \& Lee, Y. H. (2011). The Mayan activity: A way of teaching multiple quantifications in logical contexts. Problems, Resources, and Issues in Mathematics Undergraduate Studies, 21(8), 685-698.

Tall, D., \& Vinner, S. (1981). Concept image and concept definition in mathematics with particular reference to limits and continuity. Educational Studies in Mathematics, 12(2), 151-169.

Zandieh, M., \& Rasmussen, C. (2010). Defining as a mathematical activity: A framework for characterizing progress from informal to more formal ways of reasoning. The Journal of Mathematical Behavior, 29(2), 57-75. 


\section{Chapter 4: The Role of Brokering as Students Engage in Mathematical Statement Writing}

Abstract: Students' challenges with mathematical language presents an added complexity with engaging students in the disciplinary practices that rely on language such as defining or conjecturing. In this paper, I investigate the role of brokering, pedagogical content tools, and intellectual need in supporting students to refine their language when writing mathematical statements. I offer three examples in which a pair of students engaged in writing a defining property for a sequence that was unbounded above, writing a conjecture that is equivalent to the Archimedean Property of real numbers, and writing the negation of their Archimedean Property. I conclude with a conceptual framework for broking by leveraging pedagogical content tools and intellectual needs to support students in progressive mathematizing.

Keywords: Brokering, pedagogical content tools, intellectual need, disciplinary practice, mathematical statements

\section{Introduction}

Researchers have argued that undergraduate mathematics students in proof-based courses should engage in mathematical practices that parallel the activity of mathematicians (e.g. Larsen \& Zandieh, 2008; Melhuish et al., 2020; Rasmussen et al., 2015). Symbolizing and formalizing to construct definitions or theorems is part of mathematicians' work (Melhuish et al., 2020). That is, mathematicians represent mathematical concepts by using notation and symbols in such a way that transforms an informal mathematical idea to its conventional, valid, and logical form. In comparison to 
mathematicians, students face an added challenge when writing mathematical statements: formal mathematical language that is used to convey the mathematical idea is challenging for students (Dawkins \& Roh, 2019; Dubinsky \& Yiparaki, 2000, Lew \& Mejía-Ramos, 2015, 2019; Schleppegrell, 2007).

Mathematical statements often include complex quantification structures and grammatical subtleties, which are especially challenging for students (Dawkins \& Roh, 2019; Dubinsky \& Yiparaki, 2000). In Chapter 2, I reinterpreted Dubinsky and Yiparaki's (2000) game that was designed to support students in learning about statements with multiple quantifiers as how one conventionally interprets such statements. I will illustrate the conventional interpretations of AE statements (those in the form 'for all $x \in X$, there exists $y \in Y$ such that $R(x, y)$ ') and EA statements (those in the form 'there exists $y \in Y$ such that for all $x \in X, R(x, y)$ ') with two examples. Consider the following two defining properties. The first defines a sequence that is unbounded above and second defines a sequence that is bounded above:

(AE statement) For all $M \in \mathbb{R}$, there exists $n \in \mathbb{N}$ such that $a_{n}>M$. (EA statement) There exists $M \in \mathbb{R}$ such that for all $n \in \mathbb{N}, a_{n} \leq M$.

The conventional interpretation of the AE statement is that the reader can iteratively pair $M \in \mathbb{R}$ with a $n \in \mathbb{N}$ such that the following is true about the $M-n$ pairs: $a_{n}>M$. That is, the interpreter selects $M_{1} \in \mathbb{R}$ and then finds a $n_{1} \in \mathbb{N}$ such that $a_{n_{1}}>$ $M_{1}$, then selects $M_{2} \in \mathbb{R}$ and finds a $n_{2} \in \mathbb{N}$ such that $a_{n_{2}}>M_{2}$, and so on. This process can continue for every member of the set of real numbers. That is, no matter what real number $M$ the reader selects, they can always find a sequence term (defined by a natural 
number $n$ ) that is greater than the real number. Alternatively, the EA statement claims that the reader can to find one $M^{*} \in \mathbb{R}$ such that $a_{n} \leq M^{*}$ is satisfied for every $n \in \mathbb{N}$. That is, the reader can find one real number $M^{*}$ such that no matter what sequence term (defined by a natural number $n$ ) is selected, it will be less than or equal to the real number.

Chapter 2 and Chapter 3 offered examples of students using unconventional language as they engaged in writing mathematical statements. For instance, I presented a case of a pair of students defining what we called "Prime Sequence" in Chapter 2. Prior to writing the definition, the students agreed that a Prime Sequence was one that contained all the prime numbers. Conventionally, this concept could be defined by the following property: for all prime numbers $p$, there exists a natural number $k$ such that $a_{k}=p$. In contrast, the pair of students offered the following: there exists an input $k$ which maps to a prime number for all prime numbers in $\mathbb{P}$. In Chapter 2, I offer my interpretation of the students' statement to be that there is an index value where a given

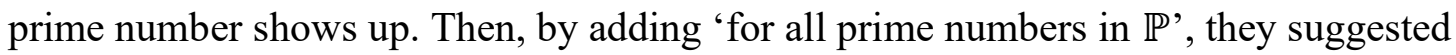
that this has to be the case for every prime number. This statement is unconventional in a number of ways, one of which is that it uses an existential quantifier before the universal quantifier.

Rasmussen and Marrongelle (2006) explain that, "As the person who knows the discipline, a teacher has the obligation of enculturating students into the discourse and conventional representational forms of the broader community while still honoring and building on students' contributions" (p. 395). In this study, I investigate how a teacher 
might enculturate students into using mathematical language as the students engage in writing mathematical statements in such a way that builds on their thinking. For instance, how might a teacher build on the students' thinking about Prime Sequences to refine their statement so that it is more consistent with the norms of the discipline? If we expect students to engage in the disciplinary practice of writing mathematical statements as in defining and conjecturing, then there is a need to understand how we might support students to refine their mathematical language. This study aims to contribute to such an understanding by drawing on data with a pair of students as they engaged in writing mathematical statements.

\section{Theoretical Background}

My investigation of instruction related to supporting students to communicate their mathematical ideas with mathematical language was guided by theory in several ways. In what follows I first discuss my view of mathematical conventions and how I view mathematical statements as notation systems that rely on multiple conventions. Then, I describe how the instructional design theory of Realistic Mathematics Education (RME) shaped the overarching instructional approach. Next, I will unpack theory that guided my investigation of instruction that supported students to effectively use mathematical language as they engaged in statement writing. This will include a discussion about the notion of intellectual need and framing an instructor as a broker.

\section{Mathematical conventions}

I take mathematical conventions to mean agreements made by the mathematics community that affect the way that its members do and write about mathematics. Hewitt 
(1999) presents a theoretical framing of mathematical convention that distinguishes between the necessary and the arbitrary. He considers a piece of knowledge to be necessary when "students can work [it] out for themselves and know [it] to be correct" ( $p$. 4) whereas an arbitrary piece of mathematical knowledge refers to what "someone could only come to know it to be true by being informed of it by some external means - whether by a teacher, a book, the internet, etc." (p. 3). Kontorovich and Zazkis (2017) exemplify these notions explaining that Hewitt would classify the sum of the interior angles in a quadrilateral is 360 degrees is a necessary piece of mathematical knowledge because students can determine this by themselves. On the other hand, Hewitt would categorize the name "quadrilateral" (or the name of any concept) as arbitrary since a learner cannot be sure whether their guess for the name is the one that is socially agreed upon.

Hewitt (1999) argues that "mathematics does not lie with the arbitrary, but is found in what is necessary" (p. 5) and stresses that students need to accept (and memorize) the arbitrary. More recently several scholars have challenged his view claiming that mathematics can also lie with the arbitrary (e.g. Larsen et al. 2020; Kontorovich \& Zazkis, 2017). Kontorovich and Zazkis argue that engaging learners in imagining plausible explanations and arguments for some choices of the mathematical community can lead the learner to non-trivial mathematical ideas and connections. For instance, they asked teachers to unpack reasons why a superscript (-1) is used to indicate reciprocals (e.g. $5^{-1}$ ) and inverse functions (e.g. $f^{-1}(x)$ ) leading the teachers to make connections between the concepts. For instance, teachers noticed that $5^{-1}$ is different than $f^{-1}(x)$ in that $5^{-1}=\frac{1}{5}$ but $f^{-1}(x) \neq \frac{1}{f(x)}$; however, both concepts involve 
"undoing": $5^{-1}$ serves as a multiplicative inverse of 5 and $f^{-1}(x)$ is an inverse function of $f(x)$.

Larsen et al. (2020) explain that viewing the arbitrary as non-mathematical is an oversimplification of notation systems that include multiple conventions because each choice has consequences and thus puts constraints on the other choices. They highlight that summation notation is an example of such a notation system in which the number below the summation conventionally represents the initial value, the number above represents the terminal value, and it is assumed that the index is incremented by 1 . Consider the following encoded sum and conventional expansion:

$$
\sum_{n=0}^{3} 2 n+1=(2 * 0+1)+(2 * 1+1)+(2 * 2+1)+(2 * 3+1)=16
$$

Once a community decides that the number below the summation (e.g. 0) represents the first input value, then the top number (e.g. 3) either can represent the number of terms (an unconventional choice summing to 9) or the terminal value of the index (the conventional choice), but not both. In this way, Larsen et al. exemplify that mathematical conventions have mathematical implications and thus advocate for engaging students in creating possible conventions and then debating the merits of the different choices.

I view mathematical statements as another example of notation systems that rely on multiple conventions. To exemplify this point, consider the definition for an unbounded above sequence: 
A sequence $\left\{a_{k}\right\}$ is called unbounded above if for all $M \in \mathbb{R}$, there exists $n \in \mathbb{N}$ such that $a_{n}>M$.

The 'for all' functions as a quantifier for $M \in R$, and refers to every member of the set of real numbers. Additionally, 'there exists' functions as a quantifier for $n \in \mathbb{N}$ and refers to at least one natural number. Interpreting the whole statement not only relies on the conventions of the individual phrases, but also how these phrases conventionally work together to convey meaning. This definition conveys that if you take a real number $M_{1}$ then you can find at least one natural number $n_{1}$ such that $a_{n_{1}}>M_{1}$. Then, if you take a real number $M_{2}$ then you can find at least one natural number $n_{2}$ such that $a_{n_{2}}>M_{2}$. And this process can continue for every member of the set of real numbers. The system of conventions of mathematical statements highlights the complexity of using mathematical language to write statements and the consequential complexities in supporting students in this way.

I side with the scholars that question Hewitt's necessary-arbitrary dichotomy. I view what he called arbitrary as being based on conventions and that many of those agreements are mathematically motivated and have mathematical implications. I maintain

that instruction should build on students' informal ideas in such a way that supports them to use conventional mathematical language rather than instruction based on memorization.

\section{Realistic Mathematics Education}

Realistic Mathematics Education (RME) is an instructional design theory for curriculum design projects that aim to guide students to reinvent concepts. Guided 
reinvention is the overarching design heuristic of such a project. The heuristic requires that instruction supports students to construct their own knowledge in a way that they come to see it "as their own, personal knowledge, knowledge for which they themselves are responsible" (Gravemeijer \& Terwel, 2000, p. 786). The word guided in guided reinvention is meant to highlight the active role that an instructor takes during the students' learning. In particular the instructor must build on students' informal ideas in such a way that supports them to construct the formal mathematics.

Guided reinvention played at least two distinct roles in my efforts to design instruction to support students to effectively use mathematical language when writing mathematical statements. First, the students were engaged in reinventing the concepts that they attempted to capture in their mathematical statement. Second, the students were guided to reinvent the mathematical language that was needed to articulate their mathematical meanings. This meant that as students engaged in writing mathematical statements, the teacher-researcher tried to inspire students to make improvements to their language in ways that was consistent with mathematical conventions. For this paper, I consider the act of trying to inspire students to make these improvements as attempts to create intellectual needs.

\section{Intellectual need}

Harel's (2008a, b) notion of intellectual need is largely consistent with the principles of Realistic Mathematics Education (RME). Harel stresses "students must engage in mathematical activities that are real to them, for which they see a purpose" (2013, p. 144). This is similar to the RME heuristic didactical phenomenology, which 
advises instructional designers to search for contexts that might evoke ways of thinking that anticipate the formal concepts that the designer intends the students to reinvent (Gravemeijer \& Terwel, 2000; Larsen, 2018). Similarly, Harel claims that students should have an intellectual need to learn what we intend to teach them. An intellectual need refers to a problem that motivates the construction of the piece of knowledge; if an individual or community possesses a piece of knowledge, then there exists a problem out of which the piece of knowledge arose (Harel, 2013). An intellectual need can be observed when a student's engagement in the problem has led them to construct the intended piece of knowledge and the student sees how the piece of knowledge resolves the problem.

Intellectual needs vary in type and scope. Harel (2013) offers five categories of intellectual needs: the need for communication, certainty, causality, computation, and structure. The need for communication is particularly relevant to the current study. Harel explains that "the need for communication occurs in mathematical practice when one is compelled to express ideas in a form and syntax that is amenable to computation by means of symbolic algebra, or when one is compelled to externalize the exact intended meaning of a concept and its logical basis" (2013, p. 6). Harel also differentiates two levels of intellectual needs: there is the main issue (i.e. global intellectual need) and problems that emerge along the way to resolve the main problem (i.e. local intellectual needs). In the current study, the global intellectual need was the students' need to communicate. In particular, the students were compelled to express their exact intended meaning of a concept and its logical basis as they engaged in writing mathematical 
statements. In order to do so, the students were encouraged to informally write their ideas in a statement. As the students and the teacher-researcher made sense of their statements, the students often experienced a local intellectual need to make refinements to their language so that it was more formal and more accurately articulated the students' idea. This study investigates how the teacher-researchers systematically created local intellectual needs to promote students to make specific refinements.

It is widely accepted among linguists that language is inherently social. Halliday comments, "language is not realized in the abstract; it is realized as the activity of people in situations" (2009, p. 18). Thus, it is no surprise that the source of the need for communication is the intrinsic desire to convey and exchange ideas (Harel, 2013). However, a need for communication is markedly different than what is called a social need. A social need is a sense of obligation to an authority, to oneself as a member of a community, or to the society in general (Harel, 2013). The distinction between a need for communication and a social need is especially important for pedagogical considerations using mathematical language because of the social nature of language.

\section{Teacher as a broker}

Rasmussen et al. (2009) draw on the communities of practice literature (e.g. Lave \& Wenger, 1991; Star \& Griesemer, 1989; Wenger, 1998) to describe the brokering role of the teacher. They explain that a broker facilitates communication and fluidity of practices between the different communities in which they hold membership (Rasmussen et al., 2009; Zandieh et al., 2017). The teacher (as a broker) is a member of a community with their students and the broader mathematical community and promotes learning by 
linking the students' practices to the broader community's practices. This present study comes from data in a laboratory setting with a pair of students and two teacherresearchers. I view the teacher-researchers as brokers, holding membership in the community with the pair of students and the broader mathematics community.

Rasmussen et al. (2009) identified three brokering moves: creating a boundary encounter, bringing participants to the periphery, and interpreting between communities. To create a boundary encounter, the broker strategically engages students in an activity that might feature differences in the practices of students and those of the broader mathematics community. A boundary encounter includes a boundary object, which refers to the "objects that serve as an interface between communities" (Rasmussen et al., 2009, p. 212). In the present study, the teacher-researchers engaged students in writing mathematical statements which exposed differences in the students' intended meanings and the conventional interpretation. I view the boundary objects as the students' statements. Additionally, the teacher-researchers, as members of the larger mathematics community, encounter the students' statements and their thinking about the statements. In this way, the teacher-researchers create a boundary encounter by engaging students in writing mathematical statements and asking questions about the statement to detect differences (and similarities) in the students' meanings and standard interpretation of the boundary object. Then, in order to support students in altering their statements so that it is more consistent with the larger mathematics community's practices, the broker can bring students to the periphery or interpret between communities. 
Bringing participants to the periphery is a brokering move that "help[s] or encourage $[\mathrm{s}]$ participants to move toward another community along a continuum" (Rasmussen et al., 2009, p. 214). In this study, this type of action brings students to the periphery of the broader mathematics community by posing a question or task that supports students in refining their statements in a way that would move them closer to convention. That is, the teacher-researchers evoke or leverage intellectual needs that compel students to refine their language in such a way that moves the students' statements closer to a valid, conventional form.

Alternatively, a broker could interpret between communities by coordinating the students' mathematics with the more conventional or formal mathematics (Zandieh et al., 2017). A move in this category is one in which a broker "facilitate[s] the understanding of one community regarding how ideas are construed, notated, related, or labeled by another community" (Rasmussen et al., 2009, p. 215). A broker that interprets between communities (re)interprets the students' thinking in terms of the standard mathematical language or notation used by the broader mathematics community and then strategically connects the students' thinking to the standard language (Rasmussen et al., 2009). Zandieh et al. suggest that this move typically occurs when "the instructor inserts notations, symbols, graphs, or diagrams, or provides other information that enable students to transcend the idiosyncrasies of their local classroom community" $(2017, \mathrm{p}$. 99). In this study, the teacher-researchers engage in interpreting between communities when they insert mathematical language as a signal for what is conventional or talked with the students about normative practices. 
I view Rasmussen and Marrongelle's (2006) notion of pedagogical content tools (PCT's) as related to Rasmussen et al.'s (2009) conceptualization of brokering. A PCT is defined as "a device, such as a graph, diagram, equation, or verbal statement, that a teacher intentionally uses to connect to student thinking while moving the mathematical agenda forward” (p. 389). I view PCT's as a device that can help facilitate brokering. Rasmussen and Marrongelle offer two types of PCT's: transformational records and generative alternatives. They define transformational records as "notations, diagrams, or other graphical representation that are initially used to record student thinking and that are later used by students to solve new problems" (p. 389). In their paper, they offer an example in which a differential equations teacher strategically recorded a student's thinking in a way that the mathematics community would recognize as the beginning of a slope field. The record (i.e. the teacher's slope field) then became a way that students reasoned about the form of the analytic expression for a differential equation and then about the space of solution functions. I view a teacher's use of transformational records as fitting under the brokering category of interpreting between communities.

Generative alternatives are especially relevant to this present study. Rasmussen and Marrongelle define this tool as "alternate symbolic expressions or graphical representations that a teacher uses to foster particular social norms for explanation and that generate student justifications for the validity of these alternatives" (2006, p. 389). Rasmussen and Marrongelle note that generative alternatives can arise in two ways, depending on who creates the alternate symbolic expression or graphical representation. First, the teacher might introduce a teacher-initiated alternative to the class with the 
intention to solicit justifications. Alternatively, a teacher might highlight a studentinitiated alternative and then engage students in a discussion about the merits of the alternative. Rasmussen and Marrongelle give an example of the latter when students were working on a problem about saltwater containing 1 pound of salt per gallon being pumped into a tank at a rate of 2 gallons per minute. The students came up with two different ideas about the rate of change - either 2 or $2 t$. The teacher capitalized on these student-initiated alternatives by engaging students in a discussion in which the students were expected to explain their reasoning and try to make sense of others' reasoning. Students not only considered the two alternatives, but also maintained social norms for advancing the mathematical idea that there is a distinction between the rate of change in a quantity and the quantity itself. In this study, I exemplify how generative alternatives can be leveraged to bring students to the periphery.

\section{Present study}

In this present study, I explore the role of brokering and intellectual need in supporting students in refining their language when writing mathematical statements. In particular, I explore: How can teachers act as brokers to support students to refine their mathematical language when writing mathematical statements? And what is the role of intellectual needs and pedagogical content tools in this endeavor? 


\section{Methods}

\section{Context and Data Collection}

This study is part of a larger design research project that is enhancing a local instructional theory in real analysis to accommodate the goal of supporting students with proof-related activity, including gaining fluency with mathematical language. The data that I present in this report comes from a laboratory design experiment (Cobb \& Gravemeijer, 2008) with Chloe and Gabe. The primary design goal for working with Chloe and Gabe was to refine tasks that supported students in reinventing foundational concepts in real analysis such as the completeness property. Another research goal was to test our conjecture that the task sequence could be leveraged to support students in learning about proof-related activity. The task design drew on the instructional design theory of Realistic Mathematics Education (Gravemeijer, 1999).

The laboratory design experiments included ten 1.5-hour long teaching sessions. All of the sessions were audio and video recorded and at least one witness was present. I shared the teacher-researcher role with another researcher. During these sessions I asked Chloe and Gabe to elaborate on or clarify their thinking when needed while the other teacher-researcher focused on posing tasks to move Chloe and Gabe's thinking forward. Between teaching sessions, we reviewed what happened during the sessions and planned for the next teaching session. Chloe and Gabe were recruited from the last two courses of the calculus sequence at their university. Both students had completed a Linear Algebra class and neither had formal proof experience at the undergraduate level prior to the 
experiment. Both students typically (but not always) earned A's in their mathematics courses.

During the sessions, the students were engaged in writing mathematical statements to articulate their thinking and iteratively refined their language. This activity can be categorized as progressive mathematizing (Gravemeijer \& Doorman,1999; Rasmussen et al., 2005) in which the students progressively formalized their language to encode their thinking. The teacher-researchers treated the students' statements as boundary objects and initiated boundary encounters by making sense of the boundary object in light of the teacher-researchers' understandings of the students' thinking about the concept that they were encoding and the standard ways of writing such a statement. Then, the teacher-researchers would either (a) try to move students to the periphery by attempting to evoke and leverage a local intellectual need, which sometimes involved using generative alternatives, or (b) interpret between communities by leveraging transformational records or by selectively sharing some of the norms of the standard practices.

\section{Data Analysis}

The data analysis for this study began with creating content logs of each teaching session by watching the video recordings. The logs were a detailed chronological account of the students' activity and discourse. The content logs included both descriptions of the data that used as little inference as possible as well as initial interpretations of the data using a higher-level of inference. The descriptions and interpretations were supplemented with pictures of the students' work and transcribed excerpts that seemed especially 
important. After the end of the experiment, I reread all of the content logs to identify episodes of the students engaging in writing mathematical statements. Within these episodes, I searched for when students appeared to experience an intellectual need that promoted refining their language in their statement. Indicators that students experienced an intellectual need included students explicitly expressing being dissatisfied with wording of the statement, described a problem with their language, or refined their language in such a way that addressed a problem. Then, I attempted to understand what about the activity or discourse could explain the emergence of the need. I identified cases when the students' discourse appeared to evoke the intellectual need and other cases in which I identified instructor moves that appeared to evoke the intellectual need.

I further explored the episodes that I identified as showcasing the teacherresearchers creating intellectual needs since my focus of this study was on the brokering role of the instructor in engaging students in writing mathematical statements. To do so, I re-watched the corresponding video and categorized the teacher-researchers' moves as creating a boundary encounter, bringing students to the periphery, or interpreting between communities. I operationalized the teacher-researcher acting as a broker by creating a boundary encounter as when the teacher-researcher engaged the students in a task that led them to write down their mathematical thinking or inquired into the students thinking about their statement in such a way that might detect differences in the students' intended meaning and how the mathematics community might interpret it. I operationalized the teacher-researcher bringing students to the periphery as instructional moves that built on the students thinking to resolve a problem with their language. Additionally, I 
operationalized the teacher-researcher interpreting between communities as instructor moves that let students know how the mathematics community construes, symbolizes, or relates ideas. I also identified when the teacher-researchers used a pedagogical content tool. Indicators that the teacher-researcher used generative alternatives included times in which they highlighted (at least) one statement and an intended meaning as contrasting cases to elicit discussion about differences or inspire students to make a refinement. Whereas, indicators that the teacher-researcher used transformational records included when the teacher-researcher requested that the students encode their thinking with a particular word, variable, or phrase that was more conventional. As expected, the teacher-researchers attempted to create an intellectual need as a way to move students to the periphery. I also found that interpreting between communities was also a way to reinforce the intellectual need that was previously created when the teacher-researchers engaged in bringing students to the periphery.

In the following section, I offer three episodes with Chloe and Gabe that detail the teacher-researchers' brokering moves and corresponding intellectual needs as students engaged in writing mathematical statements. The episodes include the students writing a defining property for unbounded above, a statement equivalent to the Archimedean Property of real numbers, and the negation of their Archimedean Property. Note that part of the data presented here, and particularly a subset of the episode of Chloe and Gabe writing a statement equivalent to the Archimedean Property, also appears in Melhuish et al. (2020). In Melhuish et al.'s paper, we used the data as part of an example of students 
engaging in authentic proof activity with the corresponding analysis focusing on the students' activity, rather than the instructor's role.

\section{Results}

In the following section, I present three episodes of brokering as students engage in mathematical statement writing. Chloe and Gabe wrote a defining property for a sequence that was unbounded above in the first episode, engaged in writing a conjecture that is equivalent to the Archimedean Property of real numbers in the second episode, and engaged in writing the negation of their Archimedean Property in the third episode.

Writing a defining property for unbounded above. In the episode that follows, the students engaged in writing a definition for unbounded above. The episode begins with the teacher-researchers creating a boundary encounter by treating the students' initial definition for a sequence that had "no upper bound" as the boundary object and inquiring into the students' thinking about their statement. The statement was unconventional in that it first introduced an existentially quantified variable and then a universally quantified variable, but it also did not fit an EA framework. The teacherresearchers then engaged in bringing students to the periphery by leveraging a generative alternative that capitalized on a difference between the boundary object and the way that Gabe described the concept with an example sequence. This episode closes with the teacher-researchers interpreting between communities by using a transformational record and sharing some of the discipline's norms.

During the fifth session with Chloe and Gabe, the students aimed to define a sequence that was unbounded above. To do so, they first offered a definition for a 
sequence that was bounded above (see Figure 32, "has upper bound $a_{n} \leq k$ where $k$ is some constant real number").

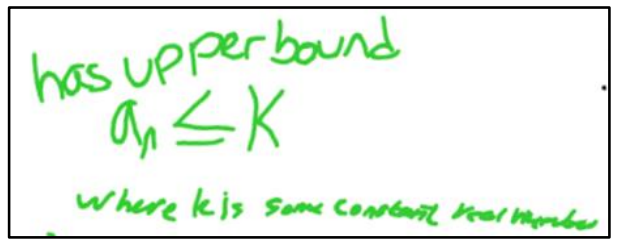

Figure 32. Chloe and Gabe's definition of "upper bound".

The teacher-researcher began creating a boundary encounter by asking "So based on that, what would it mean if it doesn't have an upper bound?" Chloe responded, "no $k$ exists" and then Gabe responded, " $a_{n}$ is greater than any, or like..." The teacherresearcher then suggested that Gabe write down his idea saying, "well give that a try and see if we can help you." Gabe's first attempt is given in Figure 33 which was leveraged by the teacher-researchers as a boundary object.

$$
\begin{aligned}
& a_{n}>k \text { for somen } \\
& \text { where } k \text { is any conseunereal number }
\end{aligned}
$$

Figure 33. Chloe and Gabe's attempt at defining "no upper bound".

The teacher-researchers continued to create a boundary encounter by inquiring into the students' thinking:

Teacher-researcher 2: Gabe, can you explain to me what you mean by 'for some $n$ ' and 'where $k$ is any constant real number'?

Gabe: So, I didn't want to have the question of for any $n$, because it's not for any $n$. If we looked at some $k$ it's basically just for some real number you can always find one that's greater, sure, but you can also find an $a_{n}$ that's greater than that. Because there is no - it will always keep being bigger. And then ' $k$ is any constant real number' - I didn't really know how to I didn't know how to encapsulate the statement into like, I guess I am just putting a variable there and saying where it could come from. 
Teacher-researcher 2: Could you maybe explain the statement using one of your sequences that doesn't have an upper bound? I still don't know if I am on the same page with what you mean by it.

Gabe: So, say there was a $k$ that was here (marks $k$ on the vertical axis and with a corresponding horizontal dotted line, see Figure 34). There are some $a_{n}$ 's that are greater than it (pointing to the sixth term) and there are some that are less, but for some $a_{n}$ 's it is greater than $k$. And we can pick another $k$ and we can also find an $a_{n}$ that is still greater.

Teacher-researcher 2: So, it works for any $k$ ?

Gabe: Yeah.

Teacher-researcher: So, no matter what $k$ you pick there is going to be something that is bigger than it.

(Gabe nods.)

Teacher-researcher: So, what do you think about that, Chloe, the no upper bound and the way it is written? Would you tweak it in any way?

Chloe: No, I don't think so.

Teacher-researcher: Alright so if we read what it says 'no upper bound, so $a_{n}$ is greater than $k$ for some $n$ where $k$ is any constant real number'. So, it sounds like there are multiple $k$ 's happening with the thing you were describing before.

Gabe: Right, so for like this what it is any $k$ (emphasizing 'any'). It's any real number. Teacher-researcher: I am wondering about the $n$. The $n$ in the thing, is the $n$ the same $n$ no matter which of the $k$ 's you're talking about?

Gabe: No. It's like not all but some of the outputs will be greater.

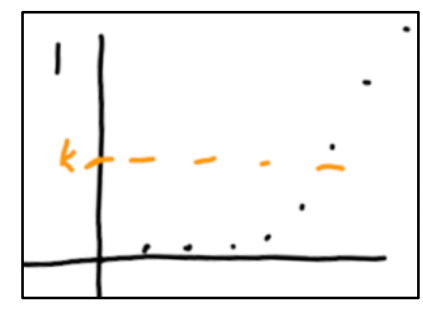

Figure 34. Gabe's illustration of an unbounded above sequence.

In the above segment, the teacher-researchers created a boundary encounter to gain an understanding of the students' meanings with the proposed "no upper bound" definition. The request to clarify what Gabe meant by 'for some $n$ ' and 'where $k$ is any constant real number' revealed that Gabe did not seem sure how to describe $k$ in a way 
that represented the concept that he was imagining and was not convinced that the statement conveyed his intended meaning. He explained, "And then ' $k$ is any constant real number' - I didn't really know how to I didn't know how to encapsulate the statement into like, I guess I am just putting a variable there and saying where it could come from." This seems to suggest that Gabe experienced an intellectual need to refine his language. The teacher-researchers' follow-up questions then supported the teacherresearchers to make sense of Gabe's intended meaning. In particular, Gabe described dynamic pairings of a real number $k$ and a sequence term $a_{n}$ such that $a_{n}>k$. He first explained he can pick a $k$ and then "for some $a_{n}$ 's it is greater than $k$. And we can pick another $k$ and we can also find an $a_{n}$ that is still greater." He then to confirmed that "there are multiple $k$ 's happening".

Note, Gabe's intended meaning is consistent with the conventional interpretation of the following property: for all $k \in \mathbb{R}$, there exists $n \in \mathbb{N}$ such that $a_{n}>k$. The boundary encounter illuminates a difference in the intended meaning and how the mathematics community might interpret the boundary object. The boundary object first introduces an existentially quantified variable $(n)$ and then a universally quantified variable $(k)$. While the boundary object does not quite fit the EA framework since both quantifiers are provided after the relation $a_{n}>k$, the mathematics community might find the boundary object ambiguous or interpret it as there exists some $n^{*} \in \mathbb{N}$ such that $a_{n^{*}}>k$ for every $k \in \mathbb{R}$. In what follows, the teacher-researchers moved the students to the periphery by leveraging a generative alternative to evoke an intellectual need to restructure their statement. 
Teacher-researcher: So is there any way to write it so that... because when you were telling the story you started with the $k$. But when I read this the $n$ is introduced before the $k$. Like 'for some $n$ ' and then it's only until after that do you say that $k$ is any real number. It feels like it's not totally in the same order. Like when you were talking over there (pointing to the example in Figure 34) that made sense to me - pick a $k$, you can find one that is above and if you keep doing that then it works. But I'm not sure that is quite saying the same thing just because it looks like you are introducing $n$ first.

Gabe: So, in this case kind of switch it around.

Teacher-researcher: Yeah. Is there a way to switch it around so that it is more obvious that you're talking about this story that you were telling over there?

(Crosstalk about saving data)

Gabe: I'm thinking in not mathy terms that 'for any real number, there is an $a_{n}$ that is greater.'

Teacher-researcher: Can you write that? Literally what you just said.

Gabe: Yeah. (writes: for any real number there is an $a_{n}$ that is greater.)

During the above exchange, the teacher-researcher leveraged a generative alternative to focus the students' attention on the structure of the boundary object in comparison to Gabe's description. In particular, the teacher-researcher capitalized on Gabe's description by pointing out that he started with $k$ but the proposed definition first described $n$. The teacher-researcher casted Gabe's description as an alternative structure and requested that the students alter the definition so that it was consistent with Gabe's explanation ("switch it around so that it is more obvious that you're talking about this story that you were telling"). Note that fulfilling this request was not only to simply please the teacher-researcher. Gabe had previously insinuated that he was not satisfied with how the statement attempted to encapsulate information about $k$. Additionally, it seemed as though the students and the teacher-researchers understood what Gabe intended to communicate with his explanation that leveraged the example. In this way, the teacher-researcher capitalized on the students' intellectual need for communication 
and advanced a structure that was more closely mapped to Gabe's intended meaning. Gabe experienced the need to reorganize the introduction of $k$ and $a_{n}$ into a logical structure that fit his explanation (i.e. intellectual need for structure) evidenced by him proposing the following statement, which the teacher-researchers used as a new boundary object:

For any real number there is an $a_{n}$ that is greater.

One difference in Gabe's meaning and the meaning that the mathematics community might construe from the boundary object is that Gabe's description relied on the variable $k$. The discussion continued with the teacher-researcher interpreting between communities by using a transformational record and sharing information about the norms of the standard practice.

Teacher-researcher: Is there a place that you can put the letter $k$ ?

(Chloe alters the statement to: for any real number, $k$ there is an $a_{n}$ that is greater, See Figure 35)

Teacher-researcher: It is pretty funny that you said 'not mathy' because this is way closer to how it is formally done than what you had before. So, you definitely made it more mathy and not less mathy.

(Laughs)

Teacher-researcher: So do you agree with that statement that it does not have any upper bound? (Question directed at Chloe)

Chloe: Yeah.

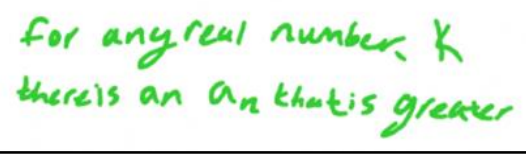

Figure 35. Refined definition of "no upper bound".

During the above interaction, the teacher-researcher used a transformational record by requesting that they use the letter $k$. This was a move that connected to Gabe's 
intended meaning and would enable the students to later use their definition.

Additionally, the teacher-researcher informed the students that this statement was more closely aligned with the standard than their previous statement ("you definitely made it more mathy and not less mathy"). The teacher-researchers ended the refining activity because it appeared that they anticipated the mathematics community interpreting the statement as the students intended. The definition in Figure 35 was the last refinement that they made for a sequence having "no upper bound".

Writing the Archimedean Property. The following episode details a series of refinements as students engaged in writing the Archimedean property of real numbers. The episode starts with the teacher-researchers engaging in creating a boundary encounter by (1) asking Chloe and Gabe to warrant a particular step in their proof, and then (2) asking follow-up questions to inquire into the students' intended meaning with the boundary object. The teacher-researcher attempted to bring the students to the periphery by using a generative alterative. The teacher-researchers asked them to consider two versions of their statement, both were true, but only one specified a variable in a way that warrants the step in the proof. The students first did not see a problem with the statements since both were true, but then experienced an intellectual need once the teacher-researcher emphasized the need to explain their step in their proof. The episode continues with the teacher-researchers interpreting between communities by first sharing a norm that reinforced an intellectual need and then used transformational records.

Chloe and Gabe engaged in writing a proof that the sequence $\left\{2^{n}\right\}$ tended to infinity during the sixth session. Their emerging proof let $k$ be a real number, let $x=$ 
$\log _{2} k$, and then "let $m>x$ where $m$ is a positive integer". The teacher-researcher began creating a boundary encounter by engaging students in warranting, asking how they knew that they could "pick a positive integer that is bigger than $x$ ". Chloe offered the following: There is always a bigger number. The teacher-researcher used this statement as the boundary object for the next part of the conversation which I take to be an attempt to move the students to the periphery by leveraging an intellectual need for communication:

Teacher-researcher: Add a little more precision to this, what kind of number is a bigger number and what is it bigger than?

(Chloe refined the statement to read: there is always a positive integer that is bigger than $x$ )

The teacher-researcher's question above leveraged the intellectual need to communicate, revealing that Chloe's exact intended meaning was unclear. This supported Chloe to refine the statement, which the teacher-researchers took to be the next boundary object: There is always a positive integer that is bigger than $x$ Next, the teacher-researcher initiated a boundary encounter by asking clarifying questions for what Chloe intended to convey with her statement:

Teacher-researcher: And what is $x$ ?

Chloe: $x$ is that one $x$ (pointing to $x=\log _{2} k$ )

Teacher-researcher: And what do we know about $x$ ?

Gabe: It is a real number.

Teacher-researcher: So, are you saying that there is always a positive integer that is bigger than that particular $x$, the one that is $\log _{2} k$ ? Or is some more general principle of numbers that you're trying to say? So, is this $x$ specific to our problem?

Chloe: It can be any chosen. I guess I meant it to be specific to our problem to answer that question that you talked about before, but like it is applicable for any $x$. 
In the above segment, the teacher-researcher continued to create a boundary encounter by asking clarifying questions (i.e. "And what is $x$ ?", "And what do we know about $x$ ?", "So are you saying that there is always a positive integer that is better than that particular $x$, the one that is $\log _{2} k$ ? Or is some more general principle of numbers that you're trying to say? So, is this $x$ specific to our problem?"). The primary purpose of these questions was to make sense of the students' thinking and detect differences between what Chloe intended and how the mathematics community would interpret the boundary object. These questions also seemed to nudge the students in considering the statement to be applicable to more than just one $x$-value $\left(x=\log _{2} k\right)$. In particular, Chloe revealed that she intended for the statement to be true for the particular $x$-value that they introduced in their proof $\left(x=\log _{2} k\right)$. Though, she explained that the statement was also true for an arbitrary $x$-value ("but like it is applicable for any $x$ "). This boundary encounter highlighted a continuity with the boundary object and the Archimedean Property of real numbers: For all real numbers $x$, there exists a positive integer $m$ such that $m>x$. In particular, the statement could be about a "more general principle of numbers" instead of only about the specific $x=\log _{2} k$. However, as written the boundary object is ambiguous because the statement does not specify $x$ to be any real number. The conversation continued with the teacher-researchers attempting to bring students to the periphery by evoking an intellectual need to specify $x$ in this way:

Teacher-researcher: So what kind of $x$ is it applicable for?

Chloe: All?

Teacher-researcher: Integers? Natural? Real? Complex?

Gabe: It has to be a real number, because if it had to be an integer than it would be straight up equal. 
Chloe: Well yeah, but there is a number that's bigger than that.

Gabe: Yeah

Chloe: It's like what the concept of infinity is. So, it doesn't matter what kind of number it is, there is a number that is bigger than it.

Teacher-researcher: So what kind of number should we say that it is in our statement of this property? If we said that 'there is always a positive integer that is bigger than $x$ where $x$ is an integer' would that be saying the same thing as saying 'where $x$ is a real number'?

Chloe: No, but they are both true.

In the above exchange, the teacher-researcher attempted to evoke an intellectual need to specify $x$ to be any real number by asking what kind of number the statement was applicable. ("So what kind of $x$ is it applicable for?") Chloe seemed focused on a need to describe a true statement, interpreting this question as "what type of number is the statement true for?' To her, specifying $x$ to be an integer versus real number were two equal choices since both versions of the statement was true. While she recognized that there was a difference in the two versions of the statement, she did not experience a need for the statement to specify $x$ to be a particular type of number. The conversation continued with the teacher-researcher leveraging a generative alternative to evoke an intellectual need:

Teacher-researcher: And which are you using here?

Chloe: It doesn't matter.

Teacher-researcher: Well let's test you on that. Write 'where $x$ is an integer'.

(Chloe altered the statement as requested: there is always a positive integer that is bigger than $x$ where $x$ is an integer)

Teacher-researcher: Now if we were somehow able to prove that true, what you just wrote, would you be able to use that to explain why you can choose an $m$ over here? Gabe: No.

Teacher-researcher 2: Why not?

Gabe: Because this isn't necessarily going to be an integer (points to $x=\log _{2} k$ ).

Chloe: Yeah, true. 
Gabe: And so it won't cover all the potential values.

Teacher-researcher: So, if you say that 'there is always a positive integer bigger than $x$ where $x$ is an integer' then that's not strong enough to use in this case.

(Chloe refined the statement to read: there is always a positive integer that is bigger than $x$ where $x$ is a number')

During this part of the discussion, the teacher-researcher leveraged generative alternatives by considering two versions of the statement, one that specified $x$ as an integer and one that specified $x$ as a real number. The teacher-researcher then evoked an intellectual need by refocusing the students to not only attend to the truth of the statement but also the need to explain the following step in their proof: "let $m>x$ where $m$ is a positive integer". The alternative that specified $x$ to be an integer was true but did not function as a warrant. The students then identified that a problem with specifying $x$ to be an integer was that "it won't cover all the potential values" of $x=\log _{2} k$. This suggests that the students understood that the version of the statement that said 'where $x$ is an integer' would not explain why they could choose a positive integer greater than $x=$ $\log _{2} k$ since $x=\log _{2} k$ wasn't necessarily an integer. As a solution, they offered a refined statement which the teacher-researchers leveraged as a boundary object for the next part of the conversation:

There is always a positive integer that is bigger than $x$ where $x$ is a number.

Note that the mathematics community might again deem this statement as ambiguous since it says 'where $x$ is a number' instead of 'where $x$ is a real number'. The teacher-researcher then inquired into the students' thinking by initiating a boundary encounter:

Teacher-researcher: Do you mean real number? Or a... 
Chloe: I don't see why it matters what number it is because there is going to be a positive integer that is larger than it.

With the teacher-researcher's question, Chloe revealed that she didn't see why there was a need to specify $x$ to be real number, and that she thought her statement (that said 'where $x$ is a number') captured her idea. The teacher-researcher then interpreted between communities by sharing a norm, which reinforced the intellectual need to specify $x$ as a real number:

Teacher-researcher: Well that's why we say real numbers when we mean that. When we mean that it doesn't matter what kind of number it is, we say real number. To do two things. To one to say that we mean it more than just naturals, or integers, or rationals, or irrationals, we mean it for all of them. That's the set that we say when it doesn't matter, we say it's true for all real numbers. It also lets them know that we are not thinking of imaginary numbers.

Chloe: Ok.

(Chloe refined the statement to read: there is always a positive integer that is bigger than $x$ where $x$ is a real number')

Teacher-researcher: Is it true for complex numbers? [inaudible]

Chloe: I don't know.

Teacher-researcher: Good answer.

(Laughs)

Teacher-researcher: Because you should probably be asking: 'Well how do I tell if a number is bigger than a complex number anyways?' 'How do I tell how big a complex number is?' There is a way, but it is not the same as what we have been doing.

In the above segment, the teacher-researcher interpreted between communities by letting Chloe and Gabe know that the mathematics community would use 'real numbers' instead of 'numbers' to represent their meaning. Notice, this reinforced the need to specify $x$ in such a way that explained why they could choose a positive integer greater than $x=\log _{2} k$. In particular, the teacher-researcher gave them the information that 'where $x$ is a number' is ambiguous and that the mathematics community uses 'real 
numbers' to clarify that $x$ could be the generic type of number that Chloe was imagining. This information revealed that (a) there is a problem with just saying $x$ is a number because it could be misinterpreted as an integer, for instance, and (b) specifying $x$ to be a real number resolved the problem as Chloe had intended. Chloe's refined statement was then used as the next boundary encounter:

There is always a positive integer that is bigger than $x$ where $x$ is a real number.

Note that the boundary object appears to be rather informal. The teacherresearchers appeared to anticipate this as potentially problematic for others to interpret the exact meaning that Chloe and Gabe intended. The conversation continued with the teacher-researcher interpreting between communities by leveraging transformational records:

Teacher-researcher: Alright, so... [reads statement under breath]. Alright, so one thing that I want to do for practice if you go back to your definition we wrote that one (pointing to a definition that the previously wrote for a sequence converging to infinity) in terms of 'for any real number there exists', so it's sort of like 'for any... there exists'. But this one over here that Chloe just wrote doesn't quite... it seems like it is trying to say the same sort of thing but not using the same kind of language. So, can we write this with saying like 'there exists' and 'for any' or 'for all'? I think that 'for all' is more common but 'for any' is also kosher.

(Chloe starts writing.)

Teacher-researcher: It's not that what you are saying is wrong, but if we kind of write them all in the same way then it is easier to see how we map them in proofs and stuff. And maybe if you use variables that would be cool too.

(Chloe and Gabe give teacher-researcher questioning look.)

Teacher-researcher: Like letters like you did before you had the $a_{x}^{\prime} s$ and the $k^{\prime} s$ (pointing to their definition of sequence converging to infinity), things like that. So, you have an $x$ for one of them but you didn't use a variable for your integer.

Chloe: Yeah.

Teacher-researcher: So maybe use a letter for both of them.

Chloe: Yeah. 
(Crosstalk while Chloe writes)

(Chloe writes: for any real number $x$ there exists a positive integer $m$ that is larger than $x)$

Chloe: Happy now?

Teacher-researcher: Well I am always happy, I am also always happy to ask for more. (Laughs)

Teacher-researcher: So, is there a mathy way to say 'is larger than'?

Gabe: Yeah.

(Cross talk while Gabe writes)

(Gabe writes: for any real number $x$ there exists a positive integer $m$ such that $m>x$ ) Teacher-researcher: Chloe, do you like that now?

Chloe: Yeah, sure.

Here, the teacher-researcher continued to connect the students' statements with a conventional version of the statement by suggesting that they use the phrases 'there exists' and 'for any' or 'for all' and then suggested that they use symbols to encode 'is larger than'. Both of these suggestions served as transformational records. With these requests, the teacher-researcher suggested that the mathematics community would use these phrases to encode the students' statements. The teacher-researchers appeared to be satisfied that the mathematics community would interpret Gabe's last refinement (for any real number $x$ there exists a positive integer $m$ such that $m>x$ ) as intended and ended the refining activity.

Writing the negation of the Archimedean Property. In the following episode I present Chloe and Gabe's refinements of their negation of their Archimedean Property. The episode starts with the teacher-researcher creating a boundary encounter by asking the students to negate their statement as a way to start proving their conjecture by way of contradiction. Their first attempt was unconventional in that it looked very similar to the Archimedean property, including its AE statement structure. The teacher-researcher 
engaged in bringing the students to the periphery by creating a need for structure by coordinating the Archimedean Property's structure, the boundary object's structure, and the students' intended meaning. The episode concludes with the teacher-researcher interpreting between communities to support students in making their final refinements.

Later in the sixth session Chloe and Gabe were tasked to negate their Archimedean Property from the previous episode (i.e. for any real number $x$ there exists a positive integer $m$ such that $m>x$ ). Negating their statement was an attempt to start proving the Archimedean Property by way of contradiction. The teacher-researcher began creating a boundary encounter by asking, "what would have to happen if it wasn't true?" Chloe responded that "there would have to be an end to numbers". She then wrote on the board " $x \geq m$ " and said "where $x$ is a number and $m$ is all numbers, all positive integers". The teacher-researcher leveraged Chloe's inequality " $x \geq m$ " as a boundary encounter.

Of course, the mathematics community would need more information about $x$ and $m$ in order to interpret the boundary object. The teacher-researcher then proceeded to interpret between communities by using a transformational record. In particular, the teacher-researcher requested that the students use similar language from the previous episode by suggesting that she write a statement using 'for all' and 'there exists'. Chloe offered the following statement, which the teacher-researchers took as the next boundary object:

For any positive integer $m$ there exists a real number $x$ where $x \geq m$. 
Chloe immediately offered an explanation, "So that says for any positive integer there is a real number where the real number is larger than the integer. (Long pause.) $\mathrm{Or}$ the real number is larger than all the positive integers" (emphasis added). With this comment, Chloe seemed to suggest that she intended the statement to convey that there was a real number that was greater than every positive integer ("Or the real number is larger than all the positive integers"). This meaning is consistent with the conventional interpretation of the standard negation. However, the mathematics community would interpret the boundary object much differently than Chloe intended. In particular, the conventional interpretation is to iteratively take $m \in \mathbb{Z}^{+}$and find a $x \in \mathbb{R}$ such that $x \geq$ $m$ until each $m \in \mathbb{Z}^{+}$has been paired. Chloe seemed to draw on their Archimedean property to construct the negation, switching the real number $x$ and the positive number $m$ and then negated the inequality so that it said $x \geq m$ instead of $m>x$. During the following, the teacher-researcher then began bringing the students to the periphery by leveraging a generative alternative to focus the students on the boundary object in comparison to Chloe's described meaning:

Teacher-researcher: So is this saying that thing you just said?

Chloe: The dis-proof?

Teacher-researcher: So you said - you wrote this and then you read it. And then you said something else.

Chloe: Oh no, I didn't read it properly.

Teacher-researcher: Ok.

Gabe: I think that for any positive integer, yeah there would exists a real number that is larger or equal to it, right?

Chloe: Yeah, I know, but the $x$ is one number. And so, we are saying for any number, like all the positive integers are less than it.

Teacher-researcher: So the question is - is what you're saying now the same thing as what you wrote?

Chloe: Ehhh - kind of. 
Gabe: I think that if it is 'for all' positive integers.

Chloe: Yeah! Yeah.

(Chloe changes it to say 'for all' instead of 'for any': For all positive integer $m$ there exists a real number $x$ where $x \geq m$.)

In the above segment, the teacher-researcher offered the boundary object and Chloe's description ("the real number is larger than all the positive integers") as generative alternatives and suggested that there was an inconsistency. I view this as an attempt to evoke an intellectual need to notice differences in the statement and Chloe's description. Chloe responded that she didn't read it properly. Recall her reading of the statement: "So that says for any positive integer there is a real number where the real number is larger than the integer." Chloe's response suggests that this did not sound to her like her intended meaning but was not yet convinced that it didn't convey that "the real number is larger than all the positive integers". On the other hand, Gabe seemed to notice a difference that was problematic, and in particular, that the statement seemed true whereas they intended to describe something that was not true - that there was a real number greater than every positive integer. He said, "I think that for any positive integer, yeah there would exists a real number that is larger or equal to it, right?" suggesting that he interpreted the statement as given any positive integer, you could find a real number that was greater or equal to the positive integer.

Again, Chloe focused on her intended meaning and reiterated that she meant to convey a different point: all the positive integers were less than one particular real number. In response, the teacher-researcher attempted to call Chloe's attention to the difference in her meaning and the boundary object. ("So the question is - is what you're 
saying now the same thing as what you wrote?") Chloe then seemed to question there was an issue with the statement, when she responded "Ehhh - kind of." Gabe attempted to resolve the problem by replacing 'any' with 'all', offering the following, which the teacher-researchers leveraged as a boundary object: For all positive integers $m$ there exists a real number $x$ where $x \geq m$. Note that this is a plausible solution if one were to take 'for all positive integer $m$ ' to mean the whole set of positive integers. In particular, one could interpret the statement as instructing the reader to first select all of the positive integers at once and then find one real number such that the real number was greater than all of the positive real numbers (see Chapter 2 for more information about this unconventional construal). Of course, this is not the standard convention.

The conversation continues with the teacher-researchers interpreting between communities by signaling that Gabe's alteration did not significantly change the statement:

Teacher-researcher: (Reading) For all positive integers there exists a real number $x$.

Chloe: The key term is 'a' real number $x$, not like..., we are talking about a specific number.

Teacher-researcher: Yeah. I think I know what you're trying to say, but I am a little suspicious whether you're actually saying that. So, if we look at what it literally says, (reading) 'for all positive integers $m$ there exists a real number $x$ where $x$ is greater than $m$.' That feels true to me.

Teacher-researcher 2: Gabe, you said previously that it seemed true, does this still feel true to you?

Gabe: Yeah, because it's like there isn't really an end to either of those groups.

In the above segment, the teacher-researcher interprets between communities, informing the students that, as a member of the mathematics community, he interpreted 
the refined statement as a true statement. This suggested that replacing the 'for any' with 'for all' did not change the meaning, at least in the way that Gabe was suggesting. This reinforced the intellectual need that Gabe experienced beforehand: the statement was true because "there isn't really an end to either of those groups," meaning the set of positive integers and the set of real numbers are unbounded above. The discussion continued with the teacher-researcher bringing the students to the periphery leveraging another generative alternative to evoke an intellectual need:

Chloe: Yeah, but you're not choosing multiple $x$ 's, you're choosing one $x$, you're choosing like 100 is $x$ and then you're saying that all the possible positive integers are less than 100. We're not going around like oh there is 101 so our $x$ is now 1000. [That] is the point that I am trying to say.

Teacher-researcher: And the point that I am trying to make is that I don't think that's what you're saying. I think that's what you're meaning and I like the thing you're meaning and I think that is what would happen if that was false. But I don't think that is captured by that. Because this seems to say very much what this says (pointing the statement their Archimedean Property). It looks like you just switched the integers and the reals.

Chloe: Yeah.

Teacher-researcher: This (pointing to their Archimedean Property) said that if you take a real number you can find a positive integer that is bigger than it.

Chloe: Yeah.

Teacher-researcher: This (pointing to the boundary object) says if you take a positive integer you can find a real number that is bigger than it. So I am like cool. So if that is how we interpret that one (their Archimedean Property), then we have to interpret that one (the boundary object) the same way.

Chloe: Yeah.

Teacher-researcher: Can we write this (the boundary object) in a different way so that it can't be interpreted like that one (the Archimedean Property)? So basically, force us to read it the way you mean it.

Chole: Ok.

(Chloe wrote: There exists a real number $x$ that is larger than all positive integers) 
In the above segment, Chloe reiterated that she intended to convey that one real number $x$ was larger than every positive integer $m$ rather than allowing for iteratively pairing between a positive integer and a real number ("We're not going around like oh there is 101 so our $x$ is now $1000 ")$. Then, the teacher-researcher confirmed that her intended meaning was what would happen if their Archimedean Property was false. Then, the teacher-researcher capitalized on the Archimedean Property and the boundary object to support the students to recognize that the structure of the boundary object caused the problem that the boundary object seemed true. In particular, the teacherresearcher explained that if the Archimedean Property conveyed that "if you take a real number you can find a positive integer that is bigger than it" then the boundary object must convey "if you take a positive integer you can find a real number that is bigger than it." This evoked an intellectual need to restructure their statement. The teacher-researcher then capitalized on another alternative - the intended meaning of the boundary object - to support the students to reconsider the structure of the boundary object in such a way that conveyed their intended meaning. That is, the teacher-researcher prompted the students to consider altering the boundary object in such a way that forced the reader to interpret the statement as intended. Chloe experienced the need to restructure the statement and resolved the problem by offering the following statement:

There exists a real number $x$ that is larger than all positive integers.

The teacher-researchers used the above statement as the next boundary object. The boundary object seems to instruct the reader to find one real number $x$ such that no matter what positive integer that is considered, the real number will be greater than the 
positive integer. A difference in the boundary object and the standard negation is that the standard typically uses symbols to encode 'that is larger than all positive integers'.

Below, Chloe recognized this difference and the teacher-researcher leveraged Chloe's comment to interpret between communities:

Chloe: There, but you don't get any mathy larger thans.

(Laughs)

Teacher-researcher: So 'There exists a real number $x$ that is larger than all positive integers'. Gabe can probably fix it with the mathy larger thans. He did it last time. Can you write that with symbols.

Gabe: The way I was thinking was, this is kind of... (long pause)

Teacher-researcher: So what you did before was you introduced a 'such that' and an inequality symbol somewhere.

Gabe: Yeah.

Teacher-researcher: Can you do a similar trick to the end of this one?

Gabe: I feel like it's a similar inequality, where it's just...

(Gabe adds 'such that $x>m$ where $m$ is any integer' below what is written so that it says 'There exists a real number $x$ that is larger than all positive integers such that $x>m$ where $m$ is any integer')

(cross talk about saving data)

Teacher-researcher: So where does what you wrote fit into the thing that Chloe started? So what does it replace?

Gabe: So all of this (pointing to 'that is larger than all positive integers')

Teacher-researcher: So maybe erase that and we will see if we are happy with it.

(Gabe writes: There exists a real number $x$ such that $x>m$ where $m$ is any integer)

In the above excerpt, the teacher-researcher followed-up with Chole's point that the boundary statement did not include "larger thans" and prompted Gabe to make the change. Gabe seemed to hesitate and then the teacher-researcher used a transformational record and suggest specific symbols from the mathematics community (i.e. 'such that' and an inequality) and suggested that their Archimedean Property was a model for how to conventionally use the symbols. When Gabe added 'such that $x>m$ where $m$ is any 
integer' to the end of the statement, the teacher-researcher suggested that instead the phrase should replace part of the statement. Then, Gabe refined the statement, which the teacher-researchers took as the next boundary object for the next part of the conversation: There exists a real number $x$ such that $x>m$ where $m$ is any integer.

Notice that the refined boundary object says 'where $m$ is any integer' whereas the standard negation uses 'for all positive integers $m$ '. Although it is not likely that the mathematics community would be confused with the statement's meaning, the teacherresearcher continued to interpret between communities by supporting them to use 'for any' instead 'where':

Teacher-researcher: That seems pretty good. I am wondering if we could do like the 'for any' thing that we did in the other one. It's looking very similar it's just reordered for these new ideas. But there is no 'where' in the (Archimedean Property). Can we do it without a where and is there some place we can put a for any.

Chloe: For any $m$.

Gabe: Yeah. I think we can move this up here. (Seemingly pointing to 'where $m$ is any integer' and suggesting moving it to after 'There exists a real number $x$ ')

Chloe: Or for any integer $m$.

Teacher-researcher: So does that go before or after 'such that'?

Chloe: It would go after 'such that'. [inaudible]

Teacher-researcher: So just put it at the very end basically?

Chloe: Yeah, it would just replace the 'where $m$ is any integer'.

(Chloe writes: There exists a real number $x$ such that $x>m$ for any integer $m$ )

Teacher-researcher: The only reason I like that a little more is because it's a little more like what you were saying. It emphasizes that it doesn't matter which integer you use, this $x$ that you picked up front is going to be bigger than all of them. Where does it too, but it's a little bit more subtle.

In the above segment, the teacher-researcher used a transformational record and suggested that the students use the phrase 'for any' and deleted 'where'. This prompted Chloe to refine the statement once more so that 'for any integer $m$ ' replaced 'where $m$ is 
any integer': There exists a real number $x$ such that $x>m$ for any integer $m$. The teacher-researchers seemed to be satisfied that the boundary object would be interpreted as intended and ended the refining activity.

\section{Discussion and Conclusion}

Brokering as students engage in writing mathematical statements is a challenging and nontrivial task. This study investigated how brokering can support students in refining their mathematical language when writing mathematical statements and the role of intellectual needs and pedagogical content tools within the brokering role. I presented three illustrative episodes of the teacher-researchers acting as brokers. In what follows, I will describe the specific brokering moves that were used by the teacher-researchers in this study in relation to intellectual needs and pedagogical content tools.

The teacher-researchers encountered the students'statements and their thinking about the statements (created a boundary encounter). The teacher-researchers treated each of the students' statements and subsequent refinements as boundary objects. The teacher-researchers made sense of the boundary objects by detecting differences between the students' intended meanings and how the mathematics community might interpret the boundary object. Once differences were detected, the teacher-researcher either (a) moved students to the periphery, or (b) interpreted between communities.

The teacher-researcher moved students to the periphery by evoking or leveraging an intellectual need. Generative alternatives were key devices that aided the teacherresearcher in evoking or leveraging intellectual needs. In the first episode, Gabe was not keen on his proposed definition of a sequence with "no upper bound". The teacher- 
researcher leveraged the need to communicate and Gabe's explanation of his intended meaning to support the students in restructuring the statement. During the second episode, the teacher-researcher used an alternative way to specify a variable in their statement as a way to evoke an intellectual need to warrant a line in their proof. In the third episode, the teacher-researcher used three alternatives: the Archimedean Property, the students' proposed statement for the negation of the property, and Chloe's description of her intended meaning. The teacher-researcher used the Archimedean Property and the students' proposed negation to evoke an intellectual need to restructure their statement and then used Chloe's description of her intended meaning to support the students in reconsidering the structure.

The teacher-researcher interpreted between communities by (a) leveraging transformational records to promote the use of more conventional language or (b) selectively disclosing normative practices of the mathematics community. This move sometimes reinforced an experienced intellectual need. In particular, while the students were writing the Archimedean Property, they experienced an intellectual need to specify a variable in such a way that their statement could be used to warrant a step in their proof. Chloe proposed a version of the statement in which the variable was specified to be 'a number'. The teacher researcher then disclosed that the mathematics community considers 'a number' to be ambiguous and that it could be misinterpreted as an integer, for example. This reinforced the need to specify the variable in such a way that warranted the step in their proof. Additionally, the third episode featured the students experiencing a need to produce a statement that captured what it would mean for the Archimedean 
Property to be false, but what they had written was true. As an attempt to resolve this issue, Gabe proposed replacing 'for any' with 'for all' seemingly suggesting using the collective construal of 'for all'. The teacher-researcher then suggested that this change did not impact how he, as a member of the mathematics community, interpreted the statement, reinforcing the need to refine the statement so that it was false.

I extracted a framework for brokering to support students engaging in disciplinary practices such as defining or conjecturing from my analysis of the three episodes. I will describe the first episode with this framework in detail. See Figure 36. The first circle represents the first boundary encounter in which the teacher-researchers treated ' $a_{n}>k$ for some $n$ where $k$ is any constant real number' as the boundary object and identified a difference in the students' intended meaning and how the mathematics community might interpret the boundary object. In particular, the mathematics community might interpret the statement differently than the students intended due to the structure of the boundary object. Then, the teacher-researcher leveraged a generative alternative, and specifically Gabe's explanation of his definition with an example, and the students' intellectual need to communicate their intended meaning to support them in restructuring the statement. This is represented by the black arrow. The second circle represents the second boundary encounter regarding the boundary object: For any real number there is an $a_{n}$ that is greater. The teacher-researcher identified that the absence of a variable might cause concern for the mathematics community. The white arrow represents the teacherresearcher using a transformational record by requesting that they use $k$ in their statement and the teacher-researcher sharing that the refined statement was "more mathy" than the 
previous. The last circle represents the boundary encounter of the last boundary object, 'for any real number, $k$ there is an $a_{n}$ that is greater' when the teacher-researcher decided to end to the refining episode since the teacher-researcher did not identify a difference between the mathematics community's expected interpretation and Chloe and Gabe's intended meaning.

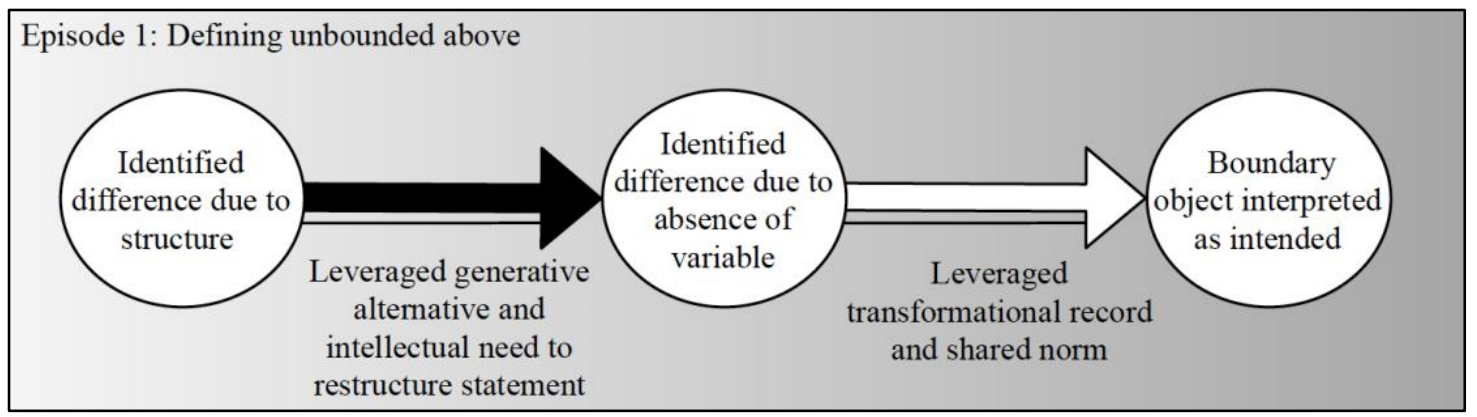

Figure 36. Brokering to support students engaging in disciplinary practices: Episode 1.

Similar diagrams can be drawn to illustrate the role of the broker in the second and third episode. See Figure 37. The circles represent boundary encounters and the arrows represent bringing students to the periphery or interpreting between communities. The arrows corresponding to bringing students to the periphery are black to indicate that the broker evoked or leveraged an intellectual need. The white and striped arrows represent the broker interpreting between communities and the striping is used to indicate that an intellectual need was reinforced. The additional lines below a subset of the arrows are used to represent that the brokering move was supported by the use of a pedagogical content tool. Additionally, the gradient shading is used to situate the brokering within the context of the local and broader mathematics community. The darker the shading, the more people that can interpret the boundary object (embedding in the boundary encounter 
circles) as the students intended. In this way, the directionality of the arrows not only point to the refinement, but also in the direction of the larger mathematics community.

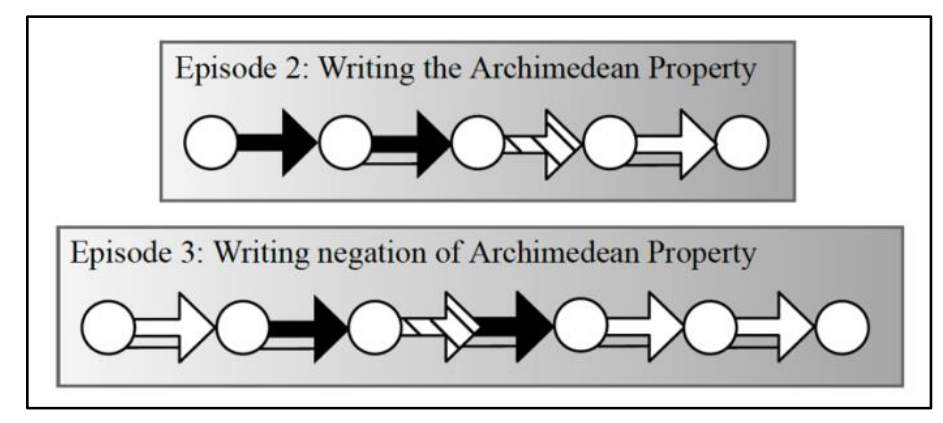

Figure 37. Brokering to support students engaging in disciplinary practices: Episode 2 and 3.

I do not intend to imply that brokering is the only way to support students in refining their mathematical language or that intellectual need doesn't play a role independent of brokering. In fact, Chapter 2 presents episodes of a pair of students engaging in defining. Many of their refinements can be attributed to their mathematical values or emerging understanding of the norms of the mathematical community. For instance, there was an instance in which one of the students suggested that they consider how one might decide whether their defining property was true or false for a particular non-example. This exploration created an intellectual need for the students to add a quantifier to their definition (from 'for $k \geq L$ ' to 'for all $k \geq L$ '). The intellectual need can be credited to the students' emerging understanding of the norms of mathematics, and specifically, that definitions should be unambiguous and that the defining property should be false for non-examples of the concept. Thus, it is possible that a corresponding diagram of an episode includes two consecutive circles with each representing the teacher encountering each refined statement. In this case, there would be no arrow between these 
two circles because the students initiated the move to the periphery themselves by reacting to an intellectual need that was not evoked by an instructor move.

I propose the following as a conceptual framework for the broker leveraging pedagogical content tools and intellectual needs in order to support students in progressive mathematizing. See Figure 38. As students engage in disciplinary practices, the artifacts of their mathematical activity can be taken as boundary objects. The teacher as a broker initiates a boundary encounter when they inquire into the students' thinking and identifies differences in the students' intended meanings and how the larger mathematics community might make sense of the boundary object. In order to address the differences, the broker can either bring students to the periphery by building on the students' thinking or interpret between communities by focusing students' attention to how the mathematics community construes, symbolizes, or relates ideas. Evoking or leveraging an intellectual need or capitalizing on generative alternatives appear to be powerful tools for the broker in bringing students to the periphery. Additionally, strategies that can support brokers to interpret between communities include leveraging transformational records or selectively disclosing normative practices of the mathematics community. If the broker is able to move students to the periphery or interpret between communities, then the students will offer a refined artifact for the broker to take as a new boundary object. The broker cycles through these moves until the broker does not detect a difference in the students' intended meaning and the anticipated mathematics community's sense making of the boundary object.

Conceptual Framework for Brokering to Support Students' Progressive Mathematizing Create Boundary Encounter: 
- Treat students' artifact of their mathematical activity as a boundary object

- Inquire into students' thinking to detect differences between students' meanings and anticipated mathematics community's construals

Bring students to periphery:

- Address difference by building on the students' thinking

Powerful strategies:

- Evoke or leverage an intellectual need

- Capitalize on generative alternatives
Interpret between communities:

- Address difference by focusing students' attention to how the mathematics community construes, symbolizes, or relates ideas

Powerful strategies:

- Leverage transformational records

- Disclose key normative practices of the mathematics community

Broker cycles through moves until broker does not detect differences between students' meanings and anticipated mathematics community's construals

Figure 38. Conceptual Framework for Brokering to Support Students' Progressive Mathematizing

While this paper focuses on brokering to support students in refining their language as they write mathematical statements, the conceptual framework is likely to be appropriate for supporting students in progressive mathematizing when they are engaged in disciplinary practices that include but not limited to, defining, conjecturing, proving, and algorithmatizing (Rasmussen et al., 2015; Rasmussen et al., 2005). I see this framework as a conceptualization of the teacher's role (as a broker) in enculturating students (as mathematics apprentices) in disciplinary practices in such a way that builds on students' contributions. 


\section{References}

Dawkins, P. C., \& Roh, K. H. (2019). Assessing the influence of syntax, semantics, and pragmatics in student interpretation of multiply quantified statements in mathematics. International Journal of Research in Undergraduate Mathematics Education, 1-22.

Dubinsky, E., \& Yiparaki, O. (2000). On student understanding of AE and EA quantification. Research in Collegiate Mathematics IV, 239-289.

Cobb, P., \& Gravemeijer, K. (2008). Experimenting to support and understand learning processes. Handbook of design research methods in education: Innovations in science, technology, engineering, and mathematics learning and teaching, 68-95.

Gravemeijer, K., \& Terwel, J. (2000). Hans Freudenthal: A mathematician on didactics and curriculum theory. Journal of Curriculum Studies, 32(6), 777-796. https://doi.org/doi:10.1080/00220270050167170

Halliday, M. A. K. (2009). Language and society (Vol. 10). Bloomsbury Publishing.

Harel, G. (2008a). DNR perspective on mathematics curriculum and instruction, Part I: focus on proving. ZDM, 4O(3), 487-500. https://doi.org/10.1007/s11858-008-0104-1

Harel, G. (2008b). A DNR perspective on mathematics curriculum and instruction, Part II: with reference to teacher's knowledge base. ZDM, 40(5), 893-907. https://doi.org/10.1007/s11858-008-0146-4

Harel, G. (2013). Intellectual need. In Vital directions for mathematics education research (pp. 119-151). Springer, New York, NY. 
Hewitt, D. (1999). Arbitrary and necessary part 1: A way of viewing the mathematics curriculum. For the learning of Mathematics, 19(3), 2-9.

Hewitt, D. (2001). Arbitrary and necessary: Part 2 assisting memory. For the learning of mathematics, 21(1), 44-51.

Hill, H. C., Ball, D. L., \& Schilling, S. G. (2008). Unpacking pedagogical content knowledge: Conceptualizing and measuring teachers' topic-specific knowledge of students. Journal for Research in Mathematics Education, 372-400.

Kontorovich, I., \& Zazkis, R. (2017). Mathematical conventions: Revisiting arbitrary and necessary. For the Learning of Mathematics, 37(1), 29-34.

Larsen, S. P. (2013). A local instructional theory for the guided reinvention of the group and isomorphism concepts. The Journal of Mathematical Behavior, 32(4), 712-725.

Larsen, S. (2018). Didactical Phenomenology: The Engine That Drives Realistic Mathematics Education. For the Learning of Mathematics, 38(3), 25-29.

Larsen, S., \& Lockwood, E. (2013). A local instructional theory for the guided reinvention of the quotient group concept. The Journal of Mathematical Behavior, 32(4), 726-742.

Larsen, S., Strand, S., \& Vroom, K. (2020). How undergraduate students think about summation (sigma) notation. Manuscript in preparation.

Larsen, S., \& Zandieh, M. (2008). Proofs and refutations in the undergraduate mathematics classroom. Educational Studies in Mathematics, 67(3), 205-216.

Lave, J., \& Wenger, E. (1991). Situated learning: Legitimate peripheral participation. Cambridge university press. 
Lew, K., \& Mejía-Ramos, J. P. (2015). Unconventional uses of mathematical language in undergraduate proof writing. In Proceedings of the 18th Annual Conference on Research in Undergraduate Mathematics Education. Pittsburgh, PA.

Lew, K., \& Mejía-Ramos, J. P. (2019). Linguistic conventions of mathematical proof writing at the undergraduate level: Mathematicians' and students' perspectives. Journal for Research in Mathematics Education, 50(2), 121-155.

Melhuish, K., Vroom, K., Lew, K., \& Ellis, B. (2020). Operationalizing authentic mathematical proof activity (AMPA) for the undergraduate classroom. Manuscript submitted for publication.

Rasmussen, C., \& Blumenfeld, H. (2007). Reinventing solutions to systems of linear differential equations: A case of emergent models involving analytic expressions. The Journal of Mathematical Behavior, 26(3), 195-210.

Rasmussen, C., \& Marrongelle, K. (2006). Pedagogical content tools: Integrating student reasoning and mathematics in instruction. Journal for Research in Mathematics Education, 388-420.

Rasmussen, C., Zandieh, M., \& Wawro, M. (2009). How do you know which way the arrows go? The emergence and brokering of a classroom mathematics practice. Mathematical representations at the interface of the body and culture, 171218.

Rasmussen, C., Wawro, M., \& Zandieh, M. (2015). Examining individual and collective level mathematical progress. Educational Studies in Mathematics, 88(2), 259-281. 
Schleppegrell, M. J. (2007). The linguistic challenges of mathematics teaching and learning: A research review. Reading \& writing quarterly, 23(2), 139-159.

Star, S. L., \& Griesemer, J. R. (1989). Institutional ecology, translations' and boundary objects: Amateurs and professionals in Berkeley's Museum of Vertebrate Zoology, 1907-39. Social studies of science, 19(3), 387-420.

Swinyard, C. (2011). Reinventing the formal definition of limit: The case of Amy and Mike. The Journal of Mathematical Behavior, 30(2), 93-114.

Wenger, E. (1999). Communities of practice: Learning, meaning, and identity. Cambridge university press.

Zandieh, M., Wawro, M., \& Rasmussen, C. (2017). An example of inquiry in linear algebra: The roles of symbolizing and brokering. Problems, Resources, and Issues in Mathematics Undergraduate Studies, 27(1), 96-124. 


\section{Chapter 5: Conclusion}

My dissertation study investigated the teaching and learning of mathematical language by supporting students to progressively formalize their language to encode their mathematical meaning. In what follows, I will summarize the three papers that comprise my dissertation, synthesize my results, and then discuss limitations and future research directions.

\section{Summary of Papers}

Students' Mathematical Language: Differences that Make a Difference. In

Chapter 2, I investigated students' thinking about the functional grammar of statements with multiple quantifiers. I offer two contributions to the researcher literature. First, I showcased grammatical complexities that influenced the way that students constructed and interpreted statements with multiple quantifiers. The research literature has reported that students' abilities to construe the conventional interpretations of statements with multiple quantifiers depends on students' understanding of the syntax, and specifically their attention to the order of the quantified variables (Dawkins \& Roh, 2019; Dubinsky \& Yiparaki, 2000). My study adds to this finding and suggests that there are other grammatical features in addition to the order of the quantifiers that impact students thinking about the statements. In particular, the students in my study considered 'for all' as different than 'for any' or 'for every' and this difference significantly affected the students' construals of AE statements. The participants considered 'there exists' as different than 'there exists at least one' which led them to non-standard interpretations of 
statements with 'there exists'. The students' interpretation activity also suggested that the grammar of 'where' is important in conveying the intended meaning of the statement.

Second, I offer a dynamical understanding of the statements themselves. Prior to this study, scholars have focused on defining features of statements with multiple quantifiers to describe static relationships between quantified variables. However, I found these descriptions were insufficient for investigating the complex ways my participants made sense of the grammar of statements with multiple quantifiers. My students attended to more than parts of the statements (e.g., the order of the quantifiers) to make sense of the statements; the students' sense making was more in line with understanding a system of interworking parts. Inspired by the students' dynamic view, I reinterpreted Dubinsky and Yiparaki's (2000) two-player game that was designed to support students in interpreting statements with multiple quantifiers as processes encoded by statements with multiple quantifiers. Consider the dynamical descriptions in Figure 39 with $R(x, y)$ representing some relation between $x$ and $y$.

\begin{tabular}{|c|c|}
\hline $\begin{array}{l}\text { AE statement: } \\
\text { For all } x \in X \text {, there exists } y \in Y \text { such that } \\
R(x, y) \text {. } \\
\text { Corresponding } A E \text { Process: } \\
\text { Iteratively take an } x \in X \text { and find a } y \in Y \\
\text { such that } R(x, y) \text { is true for the selected pair } \\
\text { and then continue this process until every } \\
\text { member of the set } X \text { has been paired with a } \\
y \in Y \text {. } \\
\text { That is, take } x_{1} \in X \text { and then find an } y_{1} \in Y \\
\text { such that } R\left(x_{1}, y_{1}\right) \text {, then select } x_{2} \in X \text { and } \\
\text { then find an } y_{2} \in Y \text { such that } R\left(x_{2}, y_{2}\right) \text {, and } \\
\text { continue this process for every member of the } \\
\text { set } X \text {. }\end{array}$ & $\begin{array}{l}\text { EA statement: } \\
\text { There exists } y \in Y \text { such that for all } x \in X, \\
R(x, y) \text {. } \\
\text { Corresponding EA Process: } \\
\text { Finding a } y^{*} \in Y \text { such that } R\left(x, y^{*}\right) \text { is true for } \\
\text { every } x \in X \text {. }\end{array}$ \\
\hline
\end{tabular}


These dynamical processes are compatible with the static relationships described by Dawkins and Roh (2019). The relationships between quantified variables can be viewed as a snapshot of the dynamical process result. The EA Process encoded in an EA statement implies that there is a one-to-every relationship (one $y \in Y$ corresponds with every $x \in X$ in such a way that $R(x, y)$ holds). Likewise, the AE Process encoded in an AE statement implies that there is an each-to-some relationship (each $x \in X$ is associated with some $y \in Y$ in such a way that $R(x, y)$ holds). How these relationships are established are through the dynamical processes described in EA and AE Processes.

\section{A Case Study of Gaining Fluency with Mathematical Language by way of}

Defining. In Chapter 3, I investigated an instructional approach for supporting students in constructing and interpreting statements with multiple quantifiers. This paper contributes to the research literature in two ways. First, it offers a proof of concept that students can be supported to reinvent $\mathrm{AE}$ and EA Processes by first engaging in defining (Zandieh \& Rasmussen, 2010). This case study offers a detailed description of the students' reinvention process.

The second contribution of this paper is progress towards articulating a local instructional theory to support the reinvention of EA and AE Processes. I used the emergent models construct from the theory of Realistic Mathematics Education to describe the evolution of the co-emerging models: EA and AE Processes. The instructional theory first promotes engaging students in situational activity for the EA Process model by engaging students in reinventing a definition of a concept that is likely to evoke an EA Process for the students. Similarly, the theory then promotes engaging 
students in situational activity for the AE Process model by engaging students in reinventing a definition of a concept that is likely to evoke an AE Process for the students. The instructional theory then supports students to engage in referential activity by engaging students in considering the grammar of the resulting set of definitions in relation to the process that they describe. The theory initiates a shift from referential to general activity by engaged students in writing an instruction manual for when to use EA and AE statements when defining. The instruction manual task promotes the modelof/model-for transition because the task requires students to make the $\mathrm{EA}$ and $\mathrm{AE}$ Processes explicit and present it in a simple enough way so that they can use the EA and AE Processes for more general mathematical activity, like defining other concepts with EA and AE Processes or interpreting other EA and AE statements.

\section{The Role of Brokering as Students Engage in Mathematical Statement}

Writing. In Chapter 4, I investigated the teacher's role in supporting students to refine their mathematical language when writing mathematical statements by drawing on the notions of brokering (Rasmussen et al., 2009), pedagogical content tools (Rasmussen \& Marrongelle, 2006), and intellectual needs (Harel, 2013). The first contribution of this paper is descriptive accounts of the teacher-researchers acting as brokers between the local community with students and the broader mathematics community to support the students in progressively formalizing their mathematical statements. In particular, the teacher-researchers first created a boundary encounter by treating the students' statement as a boundary object and then inquired into students' thinking to detect differences between the students' meanings and anticipated mathematics community's construals. 
The teacher-researcher then addressed the differences by building on the students' thinking (i.e., bringing students to the periphery) or by focusing the students' attention to how the mathematics community construes, symbolizes, or relates ideas (i.e., interpret between communities). Evoking or leveraging an intellectual need and capitalizing on generative alternatives were powerful tools for bringing students to the periphery. Leveraging transformational records and disclosing key normative practices of the mathematics community were powerful tools for interpreting between the communities. Then, the teacher-researcher cycled through these moves until they did not detect differences between students' meanings and anticipated mathematics community's construals.

I extracted a framework from my analysis of brokering to support students in refining their language in their mathematical statements. While my study focused on supporting students in engaging in writing mathematical statements, the conceptual framework is applicable for supporting students in other disciplinary practices (e.g., proving) in such a way that progressively refines students' artifacts (e.g., proofs). The main contribution of this paper is the conceptual framework for brokering to support students' progressive mathematizing. See Figure 40.

\section{Conceptual Framework for Brokering to Support Students' Progressive Mathematizing} Create Boundary Encounter:

- Treat students' artifact of their mathematical activity as a boundary object

- Inquire into students' thinking to detect differences between students' meanings and anticipated mathematics community's construals 
- Address difference by building on the students' thinking

Powerful strategies:

- Evoke or leverage an intellectual need

- Capitalize on generative alternatives
- Address difference by focusing students' attention to how the mathematics community construes, symbolizes, or relates ideas Powerful strategies:

- Leverage transformational records

- Disclose key normative practices of the mathematics community

Broker cycles through moves until broker does not detect differences between students' meanings and anticipated mathematics community's construals

Figure 40. Conceptual Framework for Brokering to Support Students' Progressive Mathematizing.

\section{Synthesis of Findings}

By considering the results of my three papers together, we can gain a better understanding of leveraging a reinvention setting to support students in learning about mathematical language, or even more broadly, to support students in learning about proof-related concepts. Here, I propose an approach in which students are supported to develop mathematical meanings, supported to progressively encode their meanings with more formal and conventional form, and then promoted to engage in more general mathematical activity. The results presented in Chapter 2 suggest that students might uses language in different ways than what is conventional (e.g., use the collective construal of 'for all'). The results of Chapter 4 offer a framing for which how the teacher can go about supporting students in progressively refining the language so that it is valid, more formal, and more conventional. 


\section{Limitations and Future Research Directions}

My dissertation study focused on studying two pairs of students thinking as they constructed and interpreted statements with multiple quantifiers. This afforded me in gaining a robust understanding of the students' thinking as well as the teacherresearchers' role in supporting their learning. I view my dissertation study as foundational for future research regarding students' thinking about statements with multiple quantifiers, instruction on supporting students in learning about statements with multiple quantifiers, and conceptualizing the teachers' role in supporting students not only in refining their mathematical language but also in supporting students in progressive mathematizing. The relatively small number of students that I worked with is a limitation for understanding (a) how common such thinking is among other students, (b) how the instructional approach might work in a classroom, and (c) additional complexities of brokering with a full classroom community. In what follows I will discuss future research avenues that build on my dissertation work.

Future research should investigate what other grammatical features impact students' use and interpretation of mathematical language. I found that engaging students in writing mathematical statements to communicate their own mathematical idea provided a rich context for investigating students' thinking about the grammar of their statements. Future researcher could use a similar approach to further explore student students' thinking about grammar, not only of statements with multiple quantifiers, but also in other forms of mathematical communication (e.g. proofs). Future research should 
also study how common it is for grammar to affect students' construction and interpretations of statements with multiple quantifiers.

Another investigation that can build from my first study is one that studies the grammar of statements with more than two quantifiers. Halliday's systemic functional grammar (Halliday \& Matthiessen, 2013) is a theory that would be an especially powerful analytic tool for studying these statements. This research would contribute to a systemic understanding of statements with more complex quantification structures, which are common in advanced mathematics definitions and theorems (e.g., definition of sequence convergence).

The larger ASPIRE research team and I are continuing to conduct series of design experiments with the instructional approach that I described in Chapter 3. One of my research goals is to further articulate a local instructional theory to support the reinvention of EA and AE Processes. One aspect of the theory that needs further development is follow-up instructional approaches to support students in refining their language as they engage in defining. My findings in Chapter 4 suggest some productive approaches. Additionally, I have learned from working with students that another potential way to support students in refining their language is to introduce a non-example of a concept that could be deemed an example based on the students' definition. Then, ask the students how they could alter the definition so that the non-example cannot be interpreted as an example of the concept. A similar task can be designed with introducing an example of a concept that could be deemed a non-example based on the students' 
definition. I intend to continue my analysis of identifying design or instructional activities that could evoke or leveraged intellectual needs to refine students language.

Future research should also investigate brokering with a classroom community. I conjecture that there will be additional complexities of brokering to investigate. For instance, in a classroom the teacher can act as a broker for the broader mathematics community, the classroom community, and communities of small groups. Future research should study how the broker coordinates the various boundary objects in such a way that supports the classroom community in progressive mathematizing. 


\section{References}

Dawkins, P. C., \& Roh, K. H. (2019). Assessing the influence of syntax, semantics, and pragmatics in student interpretation of multiply quantified statements in mathematics. International Journal of Research in Undergraduate Mathematics Education, 1-22.

Halliday, M. \& Matthiessen, C. M. (2013). Halliday's introduction to functional grammar. Routledge.

Harel, G. (2013). Intellectual need. In K. R. Leatham (Ed.), Vital Directions for Mathematics Education Research (pp. 119-151). Springer New York. https://doi.org/10.1007/978-1-4614-6977-3_6

Rasmussen, C., \& Marrongelle, K. (2006). Pedagogical content tools: Integrating student reasoning and mathematics in instruction. Journal for Research in Mathematics Education, 388-420.

Rasmussen, C., Zandieh, M., \& Wawro, M. (2009). How do you know which way the arrows go? The emergence and brokering of a classroom mathematics practice. Mathematical representations at the interface of the body and culture, 171218.

Zandieh, M., \& Rasmussen, C. (2010). Defining as a mathematical activity: A framework for characterizing progress from informal to more formal ways of reasoning. The Journal of Mathematical Behavior, 29(2), 57-75. 


\section{Appendix: Chains of signification}

\section{Chain of Signification for EA Process}

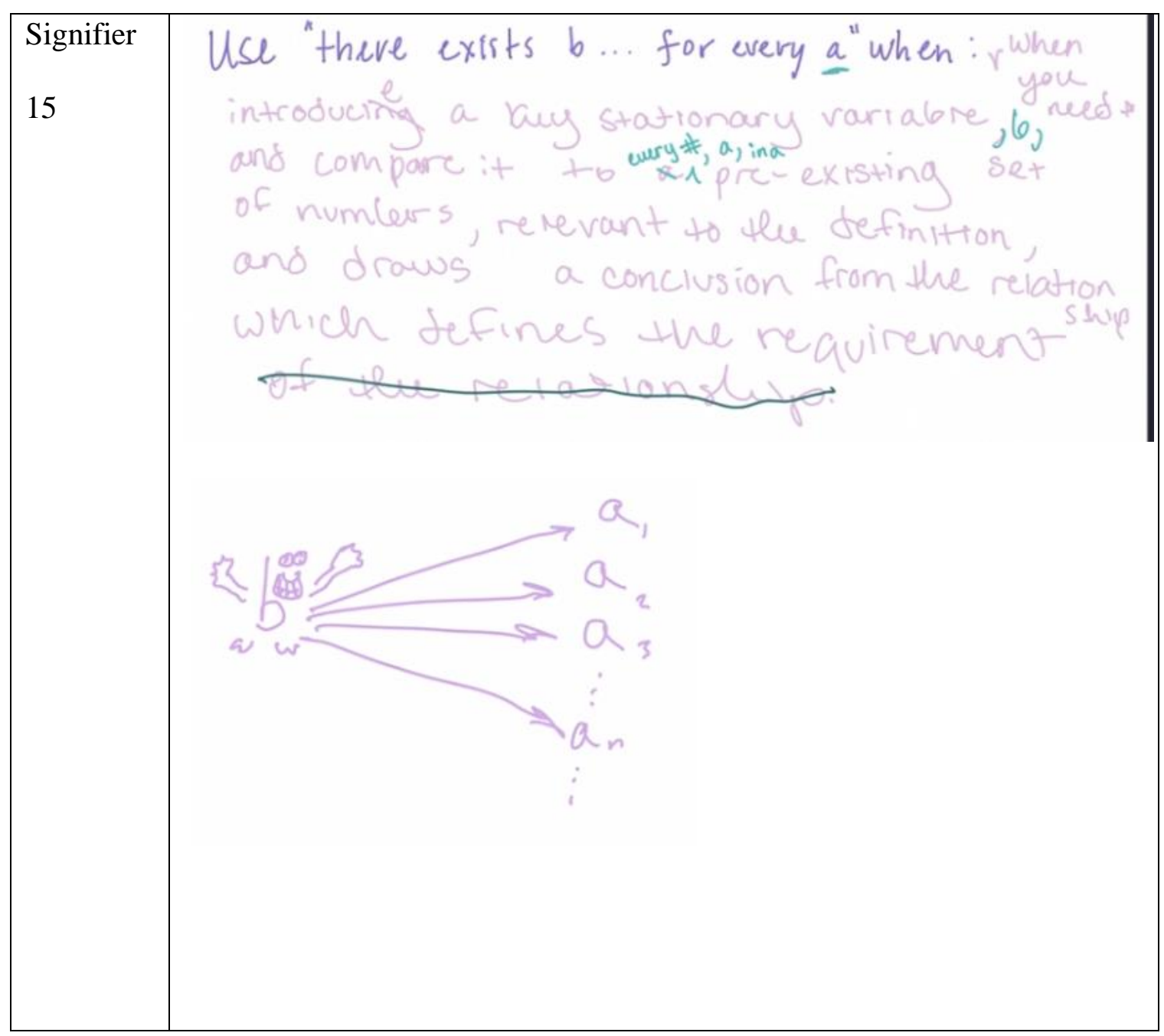




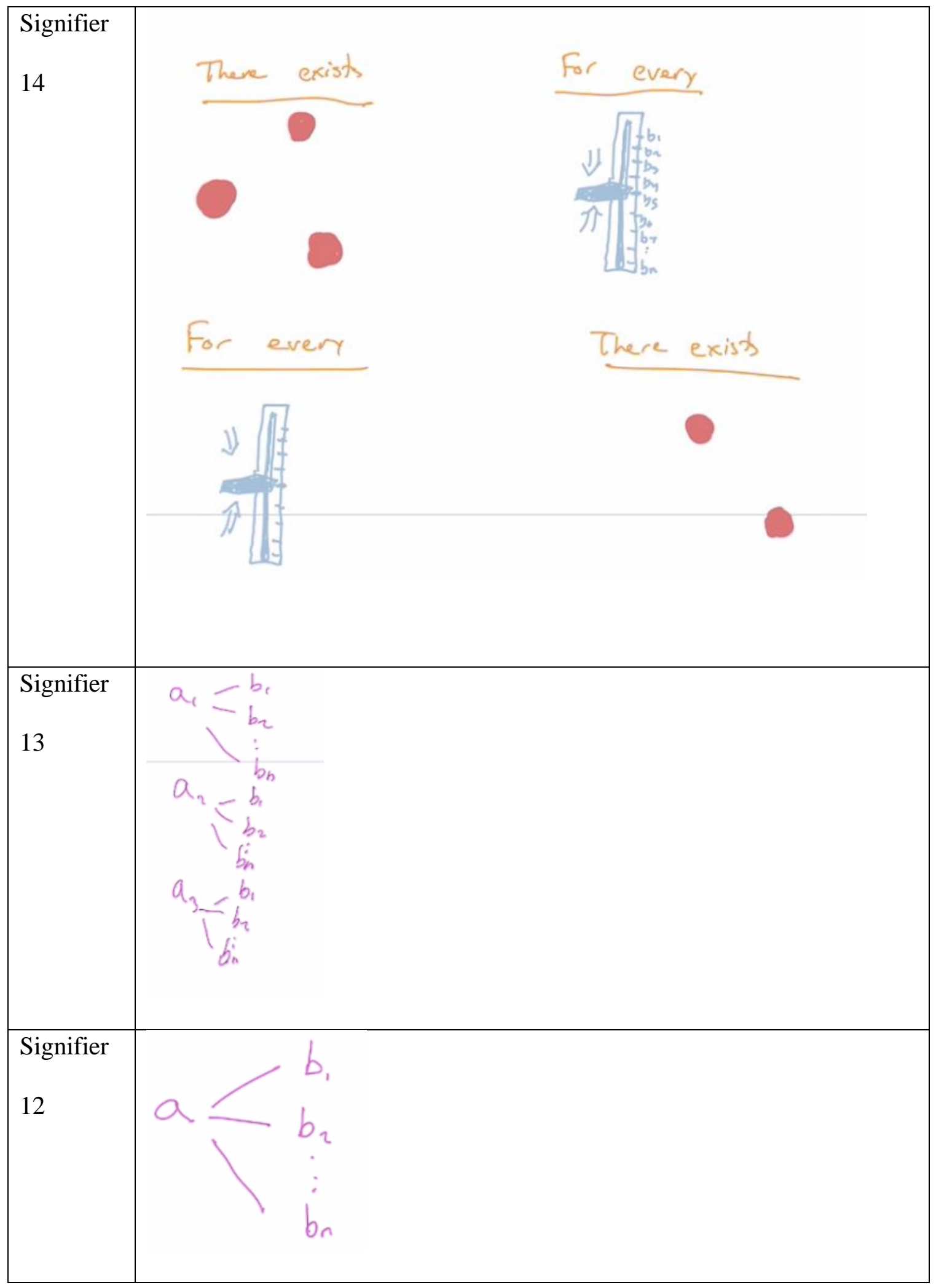




\begin{tabular}{|c|c|}
\hline $\begin{array}{l}\text { Signifier } \\
11\end{array}$ & $r_{r_{2} f} \underbrace{\vdots}_{k}$ \\
\hline $\begin{array}{l}\text { Signifier } \\
10\end{array}$ & $\frac{\vdots}{\vdots}$ \\
\hline $\begin{array}{l}\text { Signifier } \\
9 \\
\text { (PS } \\
\text { Definitio } \\
\text { n D) }\end{array}$ & 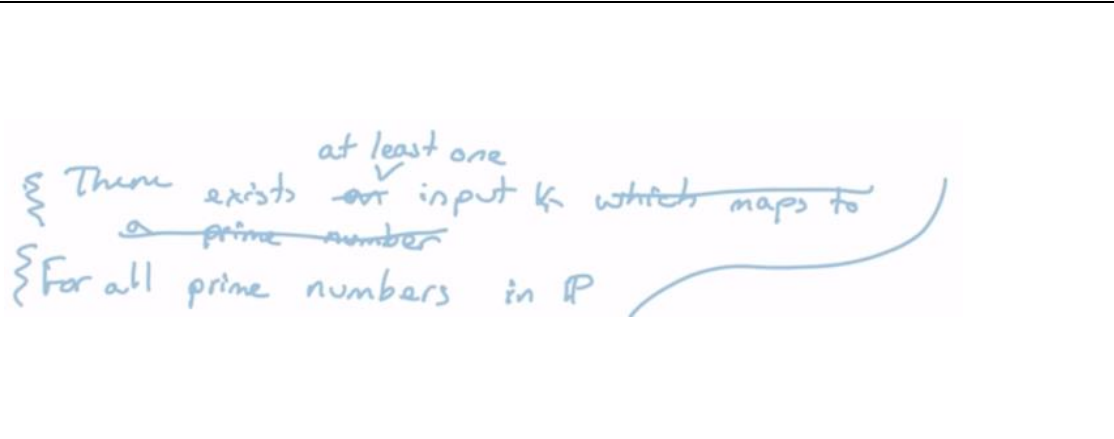 \\
\hline $\begin{array}{l}\text { Signifier } \\
8 \\
\text { (EC } \\
\text { Definitio } \\
\text { n E) }\end{array}$ & $\begin{array}{l}\text { A sequence is eventually constant if: } \\
\text { For a sequence } a_{K<} \text { there exists some natural number } L \text { where, for all } K \geq L, a_{K}=a_{K=1}\end{array}$ \\
\hline $\begin{array}{l}\text { Signifier } \\
7 \\
\text { (EC } \\
\text { Definitio } \\
\text { n D) }\end{array}$ & $\begin{array}{c}\text { A sequence is eventically constant if- } \\
\qquad \begin{array}{l}\text { For a sequence } a_{k} \text {, there exists som } \\
\text { natural nusker } L, \text { where for } k \geqslant L, a_{k}=a_{K+1}\end{array} \\
n \text {, all }\end{array}$ \\
\hline
\end{tabular}




\begin{tabular}{|c|c|}
\hline $\begin{array}{l}\text { Signifier } \\
6\end{array}$ & $\because \quad \therefore \quad \begin{array}{l}l \\
\ddots\end{array}$ \\
\hline $\begin{array}{l}\text { Signifier } \\
4 \\
\text { (EC } \\
\text { Definitio } \\
\text { n C) }\end{array}$ & $\begin{array}{l}\text { For a sequence } a_{k} \text {, there exist som } \\
\text { natural number } L \text { berond which } a_{k}=a_{k+1}\end{array}$ \\
\hline $\begin{array}{l}\text { Signifier } \\
3 \\
\text { (EC } \\
\text { Definitio } \\
\text { n B) }\end{array}$ & $\begin{array}{l}\text { For a sequence } a_{r} \text {, there exist som } \\
\text { number } L \text { berond which } a_{k}=a_{k+1}\end{array}$ \\
\hline $\begin{array}{l}\text { Signifier } \\
2 \\
\text { (EC } \\
\text { Definitio } \\
\text { n A) }\end{array}$ & $\begin{array}{l}\text { If } k \text { is very large, } \\
a_{k} \text { will equal } a_{k+1} \text {. }\end{array}$ \\
\hline Signifier & 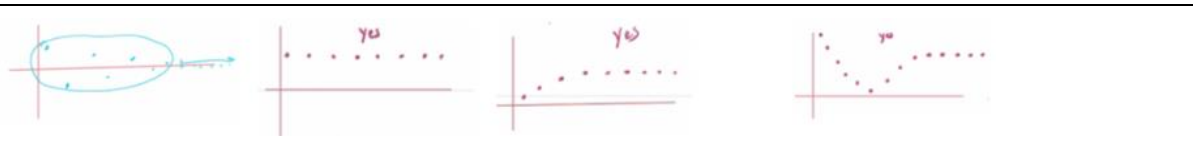 \\
\hline
\end{tabular}


Chain of Signification for AE Process

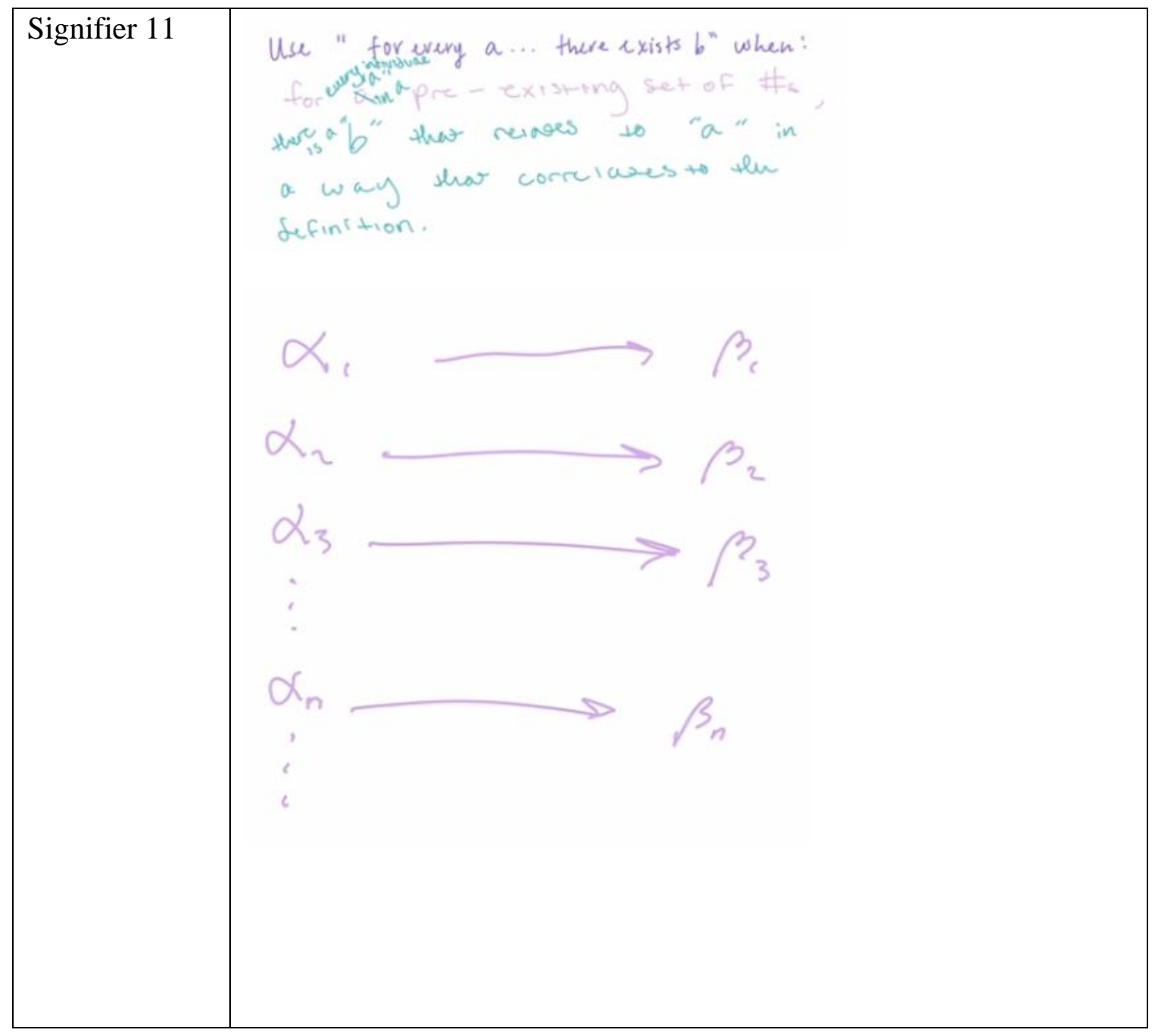




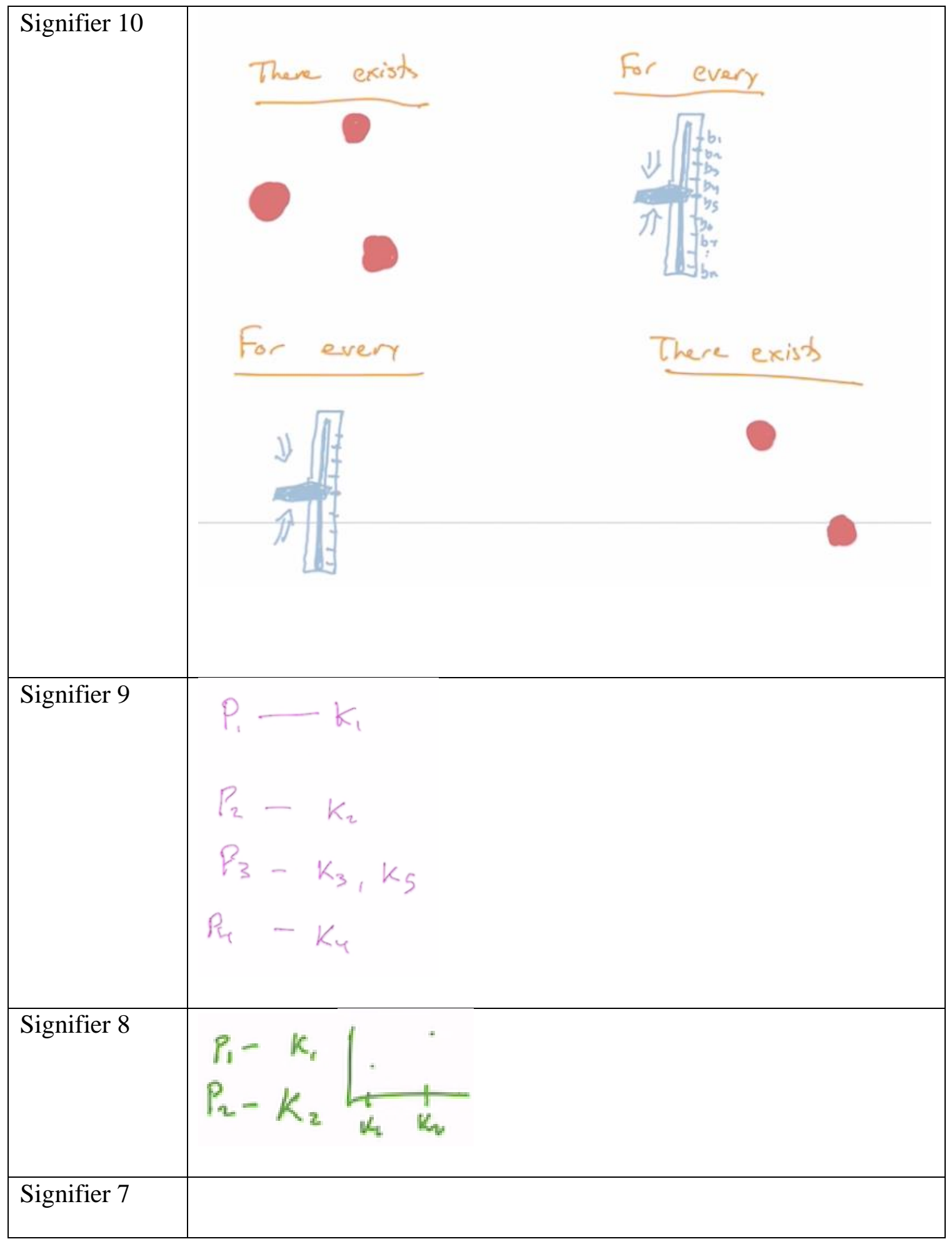




\begin{tabular}{|c|c|}
\hline $\begin{array}{l}\text { (PS Definition } \\
\text { F) }\end{array}$ & $\begin{array}{l}\text { For ecury prime numlew } p \text { in } \mathbb{P}, \\
\text { there exist at least one input } a \\
\text { where } a_{k}=p \text {. }\end{array}$ \\
\hline $\begin{array}{l}\text { Signifier } 6 \\
\text { (PS Definition } \\
\text { E) }\end{array}$ & $\begin{array}{l}\text { For all numbars in } \mathbb{P} \text {, } \\
\text { thice exists at least one } \\
\text { input } k \text { whose outpout is } \\
\text { in } \mathbb{P} \text {. }\end{array}$ \\
\hline Signifier 5 & $\begin{array}{l}\text { Thum exists at least one } \\
\text { Sorput } k \text { whrich naps to } \\
\text { \{For all prime number }\end{array}$ \\
\hline $\begin{array}{l}\text { Signifier } 4 \\
\text { (PS Definition } \\
\text { C) }\end{array}$ & $\begin{array}{l}\text { Them exists least one } \\
\text { For isput } k \text { which naps to } \\
\text { For all prime number }\end{array}$ \\
\hline $\begin{array}{l}\text { Signifier } 3 \\
\text { (PS Definition } \\
\text { B) }\end{array}$ & $\begin{array}{l}\text { Them exists an isput } k \text { which naps to } \\
\text { For all prime numbers in } \mathbb{P}\end{array}$ \\
\hline $\begin{array}{l}\text { Signifier } 2 \\
\text { (PS Definition } \\
\text { A) }\end{array}$ & $\begin{array}{l}\text { Prime sequence - } \\
\text { a prime sequinee is a sequence } \\
\text { that must consain the set of } \\
\text { prim numbers in its range. }\end{array}$ \\
\hline
\end{tabular}




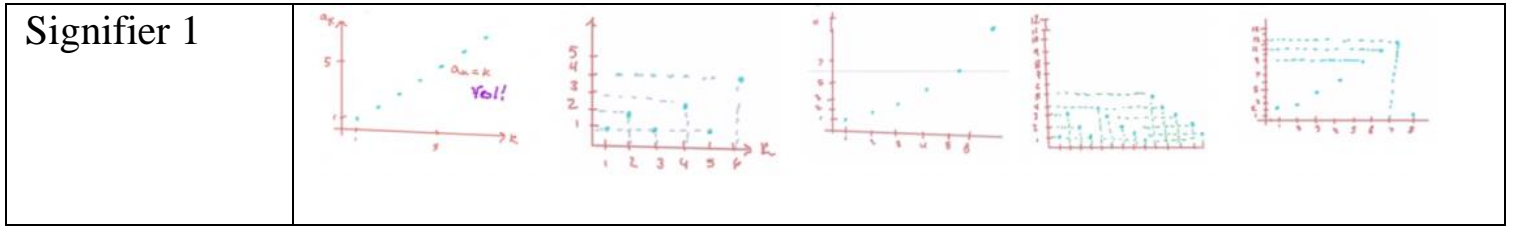

